\title{
Dearomative Indole (3+2) Reactions with Azaoxyallyl Cations - New Method for the Synthesis of Pyrroloindolines
}

\author{
Supporting Information \\ Maria C. DiPoto, Russell P. Hughes, and Jimmy Wu* \\ Department of Chemistry, Dartmouth College, Hanover, New Hampshire 03755, United States
}

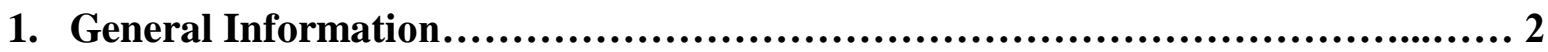

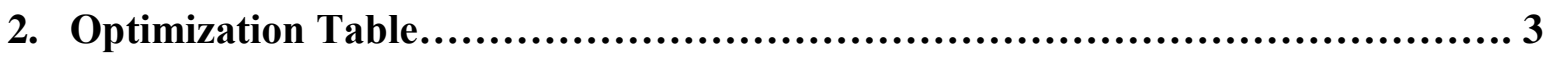

3. Experimental Procedures.......................................................... 4

a. General procedure for synthesis of $\alpha$-haloamide............................. 4

b. Synthesis of 3 -substituted indoles............................................5

c. General procedure for $(3+2)$ annulation reaction............................9

d. General procedure for $\mathrm{N}-\mathrm{O}$ bond cleavage................................. 17

e. General procedure for Protodehalogenation reaction.......................... 17

4. DFT Calculations................................................................... 18

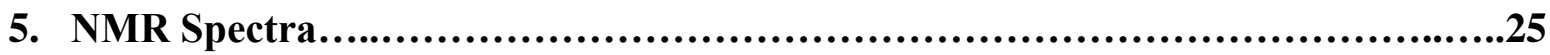




\section{General Information}

${ }^{1} \mathrm{H}-\mathrm{NMR}$ data were recorded on a Bruker Avance III $500 \mathrm{MHz}$ spectrometer (TBI probe) and a Bruker Avance III $600 \mathrm{MHz}$ spectrometer (BBFO probe) with calibration of spectra to $\mathrm{CHCl}_{3}(7.26 \mathrm{ppm})$ or $\mathrm{CH}_{2} \mathrm{Cl}_{2}(5.32 \mathrm{ppm}) .{ }^{13} \mathrm{C}-\mathrm{NMR}$ data were recorded at $125 \mathrm{MHz}$ on a Bruker Avance III $500 \mathrm{MHz}$ spectrometer (TBI probe) and at $150 \mathrm{MHz}$ on a Bruker Avance III $600 \mathrm{MHz}$ spectrometer (BBFO probe) at ambient temperature (unless otherwise noted) and are expressed in ppm using solvent as the internal standard $\left(\mathrm{CDCl}_{3}\right.$ at $77.16 \mathrm{ppm}, \mathrm{CD}_{2} \mathrm{Cl}_{2}$ at $\left.53.84 \mathrm{ppm}\right)$. Two-dimensional NMR spectra, including COSY, HSQC, HMBC and NOESY were recorded on a Bruker Avance III $500 \mathrm{MHz}$ spectrometer (TBI probe) and a Bruker Avance III $600 \mathrm{MHz}$ spectrometer (BBFO probe). Infrared spectra were recorded on a JASCO FT/IRM4100 Fourier Transform Infrared Spectrometer. Chemical shift values $(\delta)$ are expressed in ppm downfield relative to internal standard (tetramethylsilane at $0 \mathrm{ppm})$. Multiplicities are indicated as s (singlet), d (doublet), t (triplet), q (quartet), m (multiplet) and br s (broad singlet). Coupling constants are reported in hertz (Hz). Analytical thin layer chromatography (TLC) was performed on SILICYCLE pre-coated TLC plates (silica gel 60 F-254, $0.25 \mathrm{~mm}$ ). Visualization was accomplished with UV light and/or with ceric ammonium molybdate (CAM) or $\mathrm{KMnO}_{4}$ staining solutions. Flash column chromatography was performed using Biotage ${ }^{\circledR}$ Isolera System on Biotage ${ }^{\circledR}$ SNAP Ultra $10 \mathrm{~g}$ or $25 \mathrm{~g}$ columns (part No. FSUL-0442-0010 and FSUL-0442-0025). High resolution mass spectra were acquired from the Mass Spectrometry Laboratory of University of Illinois (Urbana-Champaign, IL). Melting points were determined using a Stanford Research Systems DigiMelt MPA-160 capillary melting point apparatus.

All reactions were carried out in oven or flame-dried glassware with magnetic stirring. Toluene, tetrahydrofuran (THF), diethyl ether $\left(\mathrm{Et}_{2} \mathrm{O}\right)$ and methylene chloride $\left(\mathrm{CH}_{2} \mathrm{Cl}_{2}\right)$ were dried and purified using the Glass Contour Solvent Purification System ${ }^{\circledR}$ (from Pure Process Technology, LLC) by passing the solvents through two drying columns after being purged with nitrogen. All reagents and starting materials, unless otherwise noted, were purchased from commercial vendors and used without further purification. 


\section{Full Optimization Table}

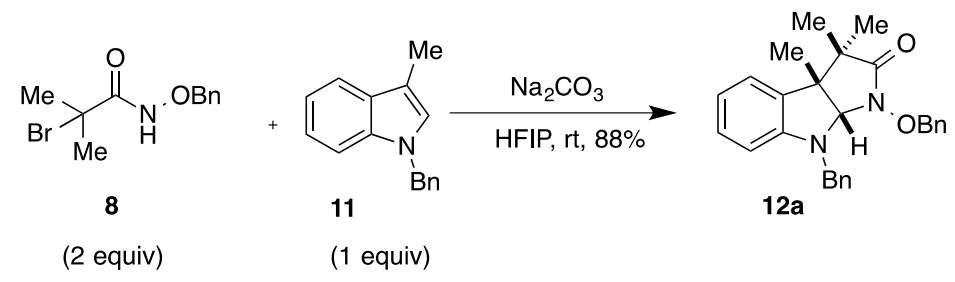

\begin{tabular}{|c|c|c|c|c|c|c|}
\hline Entry & Base & Solvent & Conc. $^{a}$ & Temp. $^{b}$ & Time $^{c}$ & Yield $^{d}$ \\
\hline 1 & $\mathrm{Na}_{2} \mathrm{CO}_{3}$ & TFE & 0.25 & $\mathrm{rt}$ & 4 & 94 \\
\hline 2 & $\mathrm{Na}_{2} \mathrm{CO}_{3}$ & TFE & 0.25 & 0 & 6 & 61 \\
\hline $3^{e}$ & $\mathrm{Na}_{2} \mathrm{CO}_{3}$ & TFE & 0.25 & $\mathrm{rt}$ & 6 & 91 \\
\hline 4 & $\mathrm{Na}_{2} \mathrm{CO}_{3}$ & THF & 0.25 & $\mathrm{rt}$ & 18 & No rxn \\
\hline 5 & $\mathrm{NEt}_{3}$ & HFIP & 0.25 & $\mathrm{rt}$ & 16 & 42 \\
\hline 6 & $\mathrm{~K}_{2} \mathrm{CO}_{3}$ & HFIP & 0.25 & $\mathrm{rt}$ & 8 & 40 \\
\hline 7 & $\mathrm{Cs}_{2} \mathrm{CO}_{3}$ & HFIP & 0.25 & $\mathrm{rt}$ & 12 & 46 \\
\hline 8 & $\mathrm{Na}_{2} \mathrm{CO}_{3}$ & HFIP & 0.25 & $\mathrm{rt}$ & 4 & 89 \\
\hline $9^{e}$ & $\mathrm{Na}_{2} \mathrm{CO}_{3}$ & HFIP & 0.25 & $\mathrm{rt}$ & 18 & 66 \\
\hline 10 & $\mathrm{Na}_{2} \mathrm{CO}_{3}$ & HFIP & 0.50 & $\mathrm{rt}$ & 6 & 39 \\
\hline 11 & $\mathrm{~K}_{2} \mathrm{CO}_{3}$ & HFIP & 0.25 & 0 & 8 & 40 \\
\hline $12^{f}$ & $\mathrm{Na}_{2} \mathrm{CO}_{3}$ & HFIP & 0.25 & 0 & $10+$ & 64 \\
\hline 13 & $\mathrm{NEt}_{3}$ & $\mathrm{LiClO}_{4} / \mathrm{Et}_{2} \mathrm{O}$ & 0.25 & $\mathrm{rt}$ & 48 & 50 \\
\hline 14 & $\mathrm{Na}_{2} \mathrm{CO}_{3}$ & $\mathrm{LiClO}_{4} / \mathrm{Et}_{2} \mathrm{O}$ & 0.25 & $\mathrm{rt}$ & 48 & No rxn \\
\hline 15 & $\mathrm{Na}_{2} \mathrm{CO}_{3}$ & $\mathrm{LiClO}_{4} / \mathrm{THF}$ & 0.25 & $\mathrm{rt}$ & 48 & 30 \\
\hline 16 & $\mathrm{Na}_{2} \mathrm{CO}_{3}$ & $\mathrm{LiClO}_{4} / \mathrm{CHCl}_{3}$ & 0.25 & $\mathrm{rt}$ & 48 & 50 \\
\hline 17 & $\mathrm{Na}_{2} \mathrm{CO}_{3}$ & $\mathrm{MeOH}$ & 0.25 & $\mathrm{rt}$ & 36 & trace \\
\hline 18 & $\mathrm{Na}_{2} \mathrm{CO}_{3}$ & $\mathrm{CH}_{2} \mathrm{Cl}_{2}$ & 0.25 & $\mathrm{rt}$ & 48 & No rxn \\
\hline 19 & $\mathrm{Na}_{2} \mathrm{CO}_{3}$ & TFE & 0.1 & $\mathrm{rt}$ & 6 & 53 \\
\hline 20 & $\mathrm{Na}_{2} \mathrm{CO}_{3}$ & TFE & 1 & $\mathrm{rt}$ & 4 & 61 \\
\hline
\end{tabular}

${ }^{a}(\mathrm{M}),{ }^{b}\left({ }^{\circ} \mathrm{C}\right),{ }^{c}(\mathrm{~h}),{ }^{d}$ (\% isolated yield), ${ }^{e} 2$ equiv indole, 1 equiv haloamide, ${ }^{f} 1$ equiv indole, 1 equiv haloamide 


\section{Experimental Procedures}

\section{a. General procedure A for synthesis of $\alpha$-haloamide ${ }^{1}$}

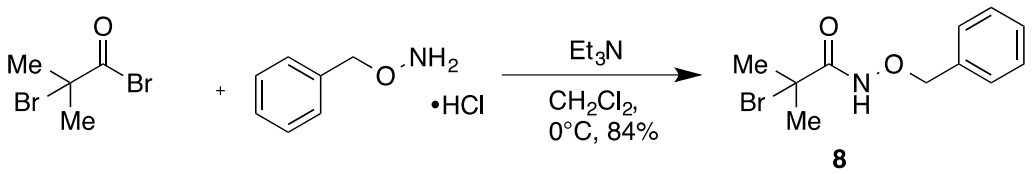

To a solution of $O$-benzylhydroxylamine $\mathrm{HCl}(2 \mathrm{~g}, 12.5 \mathrm{mmol}, 1$ eq. $)$ in dicholoromethane (50 mL), triethylamine $\left(1.75 \mathrm{~mL}, 12.5 \mathrm{mmol}, 1 \mathrm{eq}\right.$.) was added. The reaction mixture was then cooled to $0{ }^{\circ} \mathrm{C}$. Next, 2-bromo-2-methylpropanoyl bromide $(1.5 \mathrm{~mL}, 12.5 \mathrm{mmol}, 1 \mathrm{eq})$ was added dropwise to the reaction mixture. The reaction was stirred for $4 \mathrm{~h}$ at $0{ }^{\circ} \mathrm{C}$. After $4 \mathrm{~h}$, the reaction mixture was then allowed to warm to room temperature and was quenched with water. The resulting mixture was then washed with brine (x3). The organic layer was dried over anhydrous sodium sulfate, filtered, and concentrated under vacuum. The crude reaction mixture was purified by silica gel chromatography (ethyl acetate/hexanes) to give haloamide $\mathbf{8}$ as a white solid $(2.8 \mathrm{~g}, 84 \%){ }^{1}$

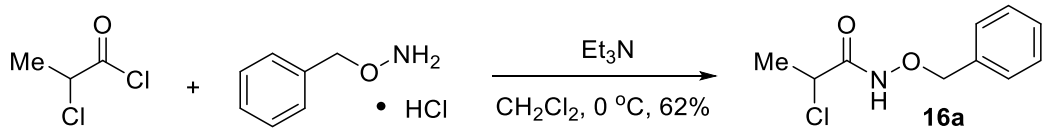

To a solution of $O$-benzylhydroxylamine $\mathrm{HCl}(2 \mathrm{~g}, 12.5 \mathrm{mmol}, 1$ eq. $)$ in dicholoromethane (50 mL), triethylamine $\left(1.75 \mathrm{~mL}, 12.5 \mathrm{mmol}, 1\right.$ eq.) was added. The reaction mixture was then cooled to $0{ }^{\circ} \mathrm{C}$. Next, 2-chloropropionyl chloride $(1.2 \mathrm{~mL}, 12.5 \mathrm{mmol}, 1 \mathrm{eq})$ was added dropwise to the reaction mixture. The reaction was stirred for $8 \mathrm{~h}$ at $0{ }^{\circ} \mathrm{C}$. After $8 \mathrm{~h}$, the reaction mixture was then allowed to warm to room temperature and was quenched with water. The resulting mixture was then washed with brine (x3). The organic layer was dried over anhydrous sodium sulfate, filtered, and concentrated under vacuum. The crude reaction mixture was purified by silica gel chromatography (ethyl acetate/hexanes) to give haloamide 16a as a white solid (1.6 g, 62\%).

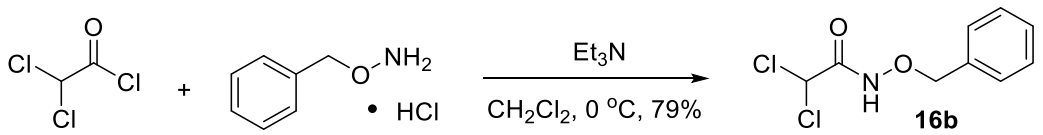

To a solution of $O$-benzylhydroxylamine $\mathrm{HCl}(2.1 \mathrm{~g}, 13.6 \mathrm{mmol}, 1$ eq.) in dicholoromethane $(54 \mathrm{~mL})$, triethylamine $\left(1.9 \mathrm{~mL}, 13.6 \mathrm{mmol}, 1\right.$ eq.) was added. The reaction mixture was then cooled to $0{ }^{\circ} \mathrm{C}$. Next, dichloroacetyl chloride $(1.3 \mathrm{~mL}, 13.6 \mathrm{mmol}, 1 \mathrm{eq})$ was added dropwise to the reaction mixture. The reaction was stirred for $4 \mathrm{~h}$ at $0{ }^{\circ} \mathrm{C}$. After $4 \mathrm{~h}$, the reaction mixture was then allowed to warm to room temperature and was quenched with water. The resulting mixture was washed with brine (x3). The organic layer was dried over anhydrous sodium sulfate, filtered, and concentrated under vacuum. The crude reaction mixture was purified by silica gel chromatography (ethyl acetate/hexanes) to give haloamide $\mathbf{1 6} \mathbf{b}$ as a white solid $(2.5 \mathrm{~g}, 79 \%){ }^{1}$

${ }^{1}$ Jeffrey, C. S.; Barnes, K.; Eickhoff, J.; Carson, C. J. Am. Chem. Soc. 2011, 133, 7688-7691. 


\section{b. Synthesis of 3-Substituted Indoles}

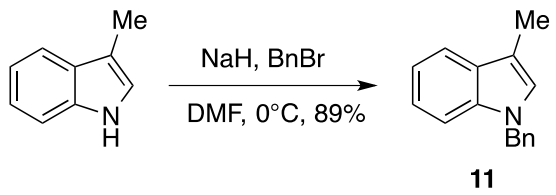

To a flame-dried flask containing 3-methylindole (500 mg, $3.9 \mathrm{mmol}, 1$ eq.), dry DMF ( $8 \mathrm{~mL}$ ) was added. The solution was cooled to $0^{\circ} \mathrm{C}$. $\mathrm{NaH}(273 \mathrm{mg}, 11.44 \mathrm{mmol}, 3$ eq. $)$ in dry DMF ( $\left.2 \mathrm{~mL}\right)$ was then added dropwise. The reaction was stirred at $0{ }^{\circ} \mathrm{C}$ for $45 \mathrm{~min}$. After $45 \mathrm{~min}$, benzyl bromide $(0.6 \mathrm{~mL}, 4.96$ mmol, 1.2 eq) was added dropwise. The reaction was allowed to warm to room temperature. Once reaction was determined to be complete via thin layer chromatographic analysis, the reaction was quenched with saturated aqueous ammonium chloride. The resulting mixture was washed with water (x3), followed by brine (x1). The organic layer was then dried over anhydrous sodium sulfate, filtered, and concentrated under vacuum. The crude reaction mixture was purified by silica gel chromatography (ethyl acetate/hexanes) to give N-benzylindole product 11 as a white solid (769 mg, 89\%). ${ }^{2}$

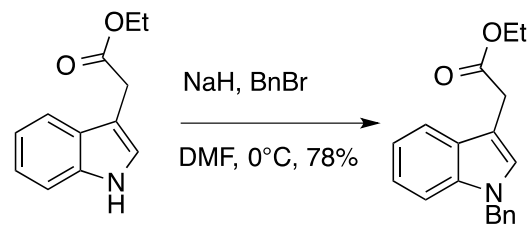

By following the general procedure B described above, a mixture of ethyl-3-indole acetate (203 $\mathrm{mg}, 1.00$ $\mathrm{mmol}, 1 \mathrm{eq}), \mathrm{NaH}(71.9 \mathrm{mg}, 3.00 \mathrm{mmol}, 3 \mathrm{eq})$, and $\mathrm{BnBr}(0.3 \mathrm{~mL}, 2.60 \mathrm{mmol})$ in DMF (10 mL) afforded N-benzyl-ethyl-3-indole acetate as a white solid $(228 \mathrm{mg}, 78 \%){ }^{3}$

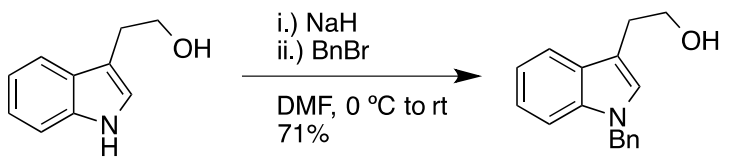

By following the general procedure B described above, a mixture of tryptophol (1g, $6.2 \mathrm{mmol}), \mathrm{NaH}$ (446 mg, $18.6 \mathrm{mmol})$, benzyl bromide $(0.8 \mathrm{~mL}, 6.8 \mathrm{mmol})$ in dry DMF $(56 \mathrm{~mL})$ afforded $\mathrm{N}$ benzyltryptophol as a yellow oil $(1.0 \mathrm{~g}, 71 \%){ }^{4}$
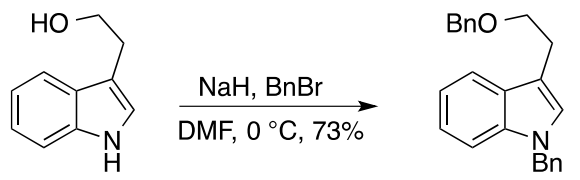

By following the general procedure B described above, a mixture of tryptophol (500 $\mathrm{mg}, 3.102 \mathrm{mmol}$ ), $\mathrm{NaH}(223 \mathrm{mg}, 9.305 \mathrm{mmol})$, benzyl bromide $(0.96 \mathrm{~mL}, 8.065 \mathrm{mmol})$ in dry DMF (30 mL) afforded Obenzyl-N-methyl tryptamine $(772 \mathrm{mg}, 73 \%) .^{5}$

\footnotetext{
${ }^{2}$ Cheng, H-G.; Lu, L-Q.; Wang, T.; Yang, Q-Q.; Liu, X-P..; Deng, Q-H.; Chen, J-R.; Xiao, W-J. Angew. Chem. Int. Ed. 2013, 52, $3250-3254$

${ }^{3}$ Alemany et al. Anales de Quimica 1974, 70, 1102-1105.

${ }^{4}$ Han, L.; Liu, C.; Zhang, W.; Shi, X-X.; You, S-L. Chem. Commun. 2014, 50, 1231-1233.

${ }^{5}$ Davis, F. A.; Melamed, J.Y.; Sharik, S.S. J. Org. Chem. 2006, 71, 8761-8766.
} 


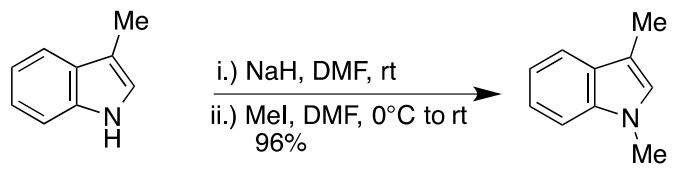

By following the general procedure B described above, a mixture of 3-methylindole (500 $\mathrm{mg}, 3.91$ mmol, 1 eq.), $\mathrm{NaH}$ (273 mg, $11.44 \mathrm{mmol}, 3 \mathrm{eq})$, and $\mathrm{MeI}(308 \mu \mathrm{L}, 4.98 \mathrm{mmol}, 1.2 \mathrm{eq})$ in dry DMF (10 $\mathrm{mL})$ afforded 1,3-dimethylindole as a colorless oil $(0.54 \mathrm{~g}, 96 \%){ }^{6}$

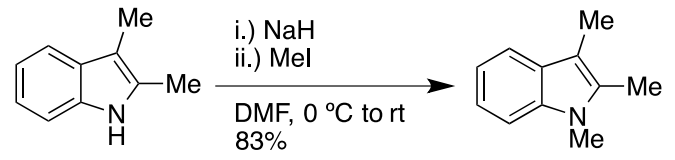

By following the general procedure B described above, a mixture of 2,3-methylindole (290 mg, $4 \mathrm{mmol}$, 1 eq.), $\mathrm{NaH}$ (288 mg, $12 \mathrm{mmol}, 3$ eq.) in dry DMF ( $2 \mathrm{~mL})$, and MeI ( $374 \mu \mathrm{L}, 6 \mathrm{mmol}, 1.5 \mathrm{eq})$ in dry DMF (10 mL) afforded 1,2,3-trimethylindole as a colorless oil (527 $\mathrm{mg}, 83 \%) .{ }^{7}$

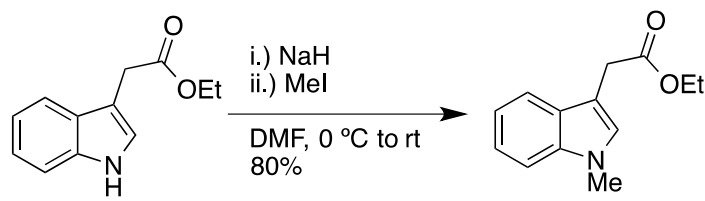

By following the general procedure B described above, a mixture of indole-3-ethyl acetate (500 mg, 2.46 mmol, 1 eq.), $\mathrm{NaH}$ (177 mg, $7.39 \mathrm{mmol}, 3$ eq.), and MeI (400 $\mu \mathrm{L}, 6.40 \mathrm{mmol}, 2.6 \mathrm{eq})$ in dry DMF (22 $\mathrm{mL})$ afforded N-methylindole-3-ethyl acetate as a colorless oil $(425 \mathrm{mg}, 80 \%){ }^{8}$

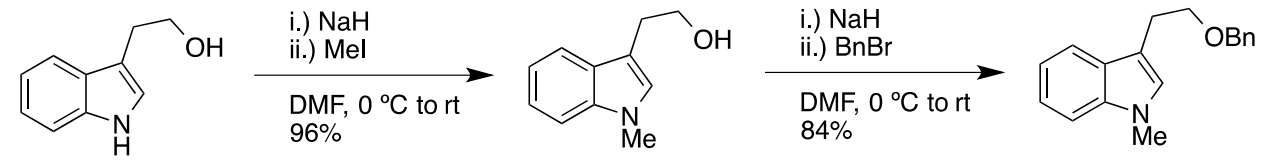

To a flame-dried flask containing tryptophol (230 mg, $1.42 \mathrm{mmol}, 1$ eq.), dry DMF (2 mL) was added. The solution was cooled to $0{ }^{\circ} \mathrm{C}$. $\mathrm{NaH}(102 \mathrm{mg}, 4.26 \mathrm{mmol}, 3$ eq.) in dry DMF (1 mL) was then added dropwise. The reaction was stirred at $0{ }^{\circ} \mathrm{C}$ for $45 \mathrm{~min}$. After $45 \mathrm{~min}$, MeI ( $\left.96 \mu \mathrm{L}, 1.56 \mathrm{mmol}, 1 \mathrm{eq}\right)$ was then added dropwise. The reaction was allowed to warm to room temperature. Once the reaction was determined to be complete via thin layer chromatographic analysis, the reaction was quenched with saturated aqueous ammonium chloride. The resulting mixture was washed with water (x3), followed by brine (x1). The organic layer was then dried over anhydrous sodium sulfate, filtered, and concentrated under vacuum. The crude reaction mixture was purified by silica gel chromatography (ethyl acetate/hexanes) to give $\mathrm{N}$-methyltryptophol (240 mg, 96\%). Next, to a flame-dried flask containing N-methyltryptophol (215 mg, $1.29 \mathrm{mmol}, 1$ eq.), dry DMF $(10 \mathrm{~mL})$ was added. The solution was cooled to $0{ }^{\circ} \mathrm{C}$. $\mathrm{NaH}(88$ $\mathrm{mg}, 3.68 \mathrm{mmol}, 3$ eq.) in dry DMF ( $2 \mathrm{~mL})$ was then added dropwise and reaction was stirred at $0{ }^{\circ} \mathrm{C}$ for $45 \mathrm{~min}$. After $45 \mathrm{~min}$, benzyl bromide $(0.4 \mathrm{~mL}, 3.20 \mathrm{mmol}, 2.6 \mathrm{eq})$ was added dropwise, and the reaction was allowed to warm to room temperature. Once the reaction was determined to be complete via thin layer chromatographic analysis, the reaction was quenched with saturated aqueous ammonium chloride.

${ }^{6}$ Klare, H. F. T.; Oestreich, M.; Ito, J-I.; Nishiyama, H.; Ohki, Y.; Tatsumi, K. J. Am. Chem. Soc. 2011, 133, $3312-3315$.

${ }^{7}$ Liu, Y.; Yao, B.; Deng, C-L.; Tang, R-Y.; Zhang, X-G.; Li, J-H. Org. Lett. 2011, 13, 1126-1129.

${ }^{8}$ Greulich, T. W.; Daniliuc, C.G.; Studer, A. Org. Lett. 2015, 17, 254-257. 
The resulting mixture was washed with water (x3), followed by brine (x1). The organic layer was then dried over anhydrous sodium sulfate, filtered, and concentrated under vacuum. The crude reaction mixture was purified by silica gel chromatography (ethyl acetate/hexanes) to give O-benzyl-Nmethyltryptamine product $(271 \mathrm{mg}, 84 \%) .^{9}$

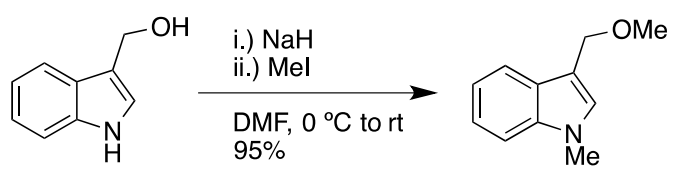

By following the general procedure $\mathrm{B}$ described above, a mixture of 3-indole methanol (500 $\mathrm{mg}, 3.4$ mmol, 1 eq.), $\mathrm{NaH}$ (245 mg, $10.2 \mathrm{mmol}, 3$ eq.), and MeI (0.55 mL, $8.84 \mathrm{mmol}, 2.6 \mathrm{eq})$ in dry DMF (43 $\mathrm{mL}$ ) afforded N,O-dimethylindole product (169 mg, $95 \%){ }^{10}$

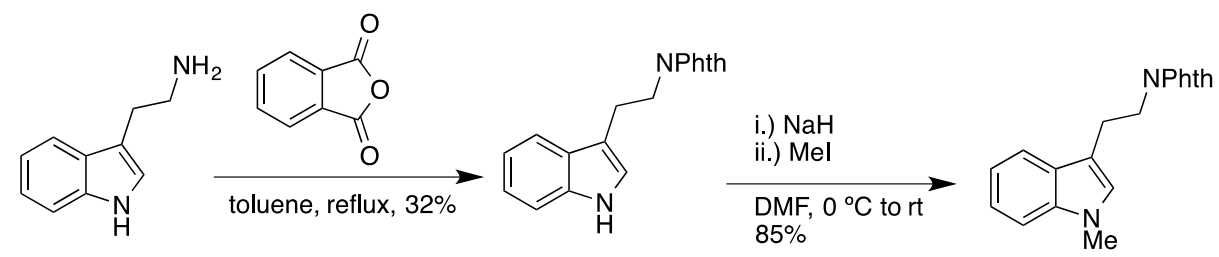

To a round bottom flask containing tryptamine $(365 \mathrm{mg}, 2.28 \mathrm{mmol}, 1 \mathrm{eq})$ in toluene $(5 \mathrm{~mL})$, phthalic anhydride was added (404 mg, $2.73 \mathrm{mmol}, 1.2 \mathrm{eq})$. The reaction was then stirred at reflux for $12 \mathrm{~h}$. Once reaction was determined to be complete via thin layer chromatographic analysis, the reaction mixture was concentrated under vacuum. The product was then recrystallized using ethyl acetate $(212 \mathrm{mg}, 32 \%)$. Then, to a flame-dried flask containing indole $\mathrm{N}^{\prime}$-phthalyltryptamine ( $35 \mathrm{mg}, 0.12 \mathrm{mmol}, 1 \mathrm{eq}$.), dry $\operatorname{DMF}(0.1 \mathrm{~mL})$ was added. The solution was cooled to $0^{\circ} \mathrm{C}$. Then, $\mathrm{NaH}(8 \mathrm{mg}, 0.24 \mathrm{mmol}, 3 \mathrm{eq}$. $)$ in dry DMF $(0.1 \mathrm{~mL})$ was added dropwise. The reaction was stirred at $0{ }^{\circ} \mathrm{C}$ for $45 \mathrm{~min}$. After $45 \mathrm{~min}$, MeI ( 7 $\mu \mathrm{L}, 0.1 \mathrm{mmol}, 1.2 \mathrm{eq})$ was added. The reaction was allowed to warm to room temperature. Once reaction was determined to be complete via thin layer chromatographic analysis, the reaction was quenched with saturated aqueous ammonium chloride. The resulting mixture was washed with water (x3), followed by brine (x1). The organic layer was then dried over anhydrous sodium sulfate, filtered, and concentrated under vacuum. The crude reaction mixture was purified by silica gel chromatography (ethyl acetate/hexanes) to give N-methyl-N'-phthalyltryptamine $(588 \mathrm{mg}, 85 \%){ }^{2}$

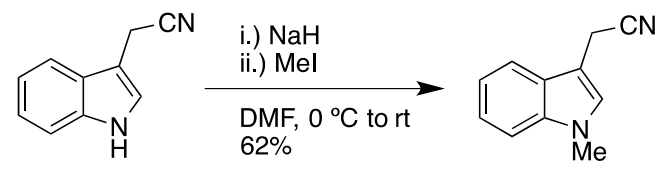

Following general procedure $\mathrm{B}$ as described above, a mixture of indole-3-acetonitrile $(1 \mathrm{~g}, 6.4 \mathrm{mmol}, 1$ eq.), $\mathrm{NaH}(200 \mathrm{mg}, 8.33 \mathrm{mmol}, 3$ eq. $)$, and $\mathrm{MeI}(0.6 \mathrm{~mL}, 9.58 \mathrm{mmol}, 1.5 \mathrm{eq})$ in dry DMF $(7 \mathrm{~mL})$ afforded N-methylindole-3-acetonitrile as a colorless oil (672 mg, $62 \%){ }^{11}$

\footnotetext{
${ }^{9}$ Yadav, J.S.; Reddy, B. V. S.; Reddy, C. S.; Kishna A. D. Tet. Lett. 2007, 41, 2029-2032.

${ }^{10}$ Lown, J. W.; Weir, G. L. Can. J. Chem. 1978, 58, 249-255.

${ }^{11}$ Morales-Rios, M. S.; Santos-Sanchez, N.F.; Mora-Perez, Y.; Joseph-Nathan, P. Heterocycles 2004, 63, $1331-1142$.
} 


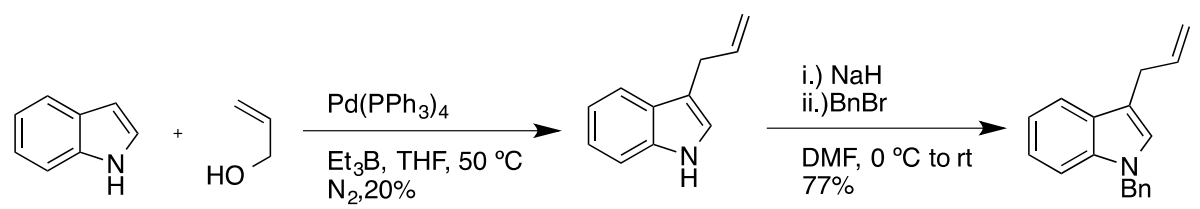

To a flame-dried flask under nitrogen containing indole (1g, $8.5 \mathrm{mmol}, 1 \mathrm{eq})$ in THF $(20 \mathrm{~mL}), \mathrm{Ph}\left(\mathrm{PPh}_{3}\right)_{4}$ (295 mg, $0.25 \mathrm{mmol}, 0.03 \mathrm{eq}$ ) was added, followed by allyl alcohol $(0.6 \mathrm{~mL}, 9.4 \mathrm{mmol}, 1.1 \mathrm{eq})$ and $\mathrm{Et}_{3} \mathrm{~B}$ ( $1 \mathrm{M}$ in hexanes, $2.5 \mathrm{~mL}, 2.5 \mathrm{mmol}, 0.3 \mathrm{eq}$ ). The reaction was stirred at $50{ }^{\circ} \mathrm{C}$. When the reaction was determined to be complete via thin layer chromatographic analysis, the reaction was diluted with ethyl acetate. The mixture was then washed with saturated aqueous sodium bicarbonate $(1 \mathrm{x})$, followed by brine (1x). The organic layer was dried over anhydrous sodium sulfate and concentrated under rotary evaporation. The resulting residue was purified by silica gel chromatography (ethyl acetate/hexanes) to give 3-allylindole (260 mg, 20\%). Next, to a flame-dried flask containing 3-allylindole (105 mg, 0.66 mmol, 1 eq.), dry DMF (1 mL) was added. The solution was cooled to $0^{\circ} \mathrm{C}$. Then, $\mathrm{NaH}(21 \mathrm{mg}, 0.87$ mmol, 1.3 eq. $)$ in dry DMF $(0.5 \mathrm{~mL})$ was added dropwise. The reaction was stirred at $0{ }^{\circ} \mathrm{C}$ for $45 \mathrm{~min}$. After $45 \mathrm{~min}$, benyzyl bromide $(63 \mu \mathrm{L}, 1.0 \mathrm{mmol}, 1.5 \mathrm{eq})$ was added dropwise. The reaction was allowed to warm to room temperature. Once reaction was determined to be complete via thin layer chromatographic analysis, the reaction was quenched with saturated aqueous ammonium chloride. The resulting mixture was washed with water (x3), followed by brine (x1). The organic layer was then dried over anhydrous sodium sulfate, filtered, and concentrated under vacuum. The crude reaction mixture was purified by silica gel chromatography (ethyl acetate/hexanes) to give N-benzyl-3-allylindole as a colorless oil $(87.5 \mathrm{mg}, 77 \%){ }^{12}$

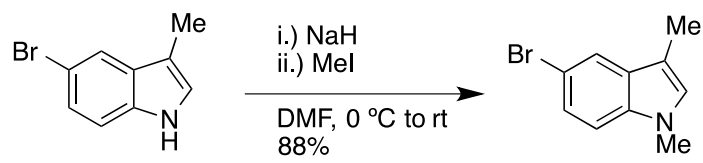

Following general procedure $\mathrm{B}$ as described above, a mixture of 5-bromo-3-methylindole (180 mg, 0.86 mmol, 1 eq.), $\mathrm{NaH}$ (62 mg, $2.58 \mathrm{mmol}, 3$ eq.), and MeI (107 $\mu \mathrm{L}, 1.72 \mathrm{mmol}, 2 \mathrm{eq})$ in dry DMF ( $2 \mathrm{~mL})$ afforded 5-bromo-1, 3-dimethylindole as a colorless oil (168 mg, $88 \%){ }^{13}$

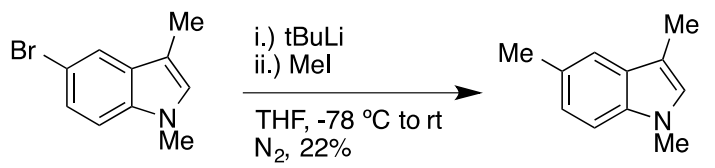

To a flame-dried round bottom flask under nitrogen containing 1,3-dimethyl-5-bromoindole (80 mg, $0.35 \mathrm{mmol}, 1 \mathrm{eq})$ in dry THF $(1.5 \mathrm{~mL})$ at $-78{ }^{\circ} \mathrm{C}, t$-BuLi in $18 \%$ hexanes $(480 \mu \mathrm{L}, 0.89 \mathrm{mmol}, 2.5 \mathrm{eq})$ was added dropwise. The reaction was stirred for $15 \mathrm{~min}$ at $-78^{\circ} \mathrm{C}$, then the reaction mixture was treated with $\mathrm{MeI}(27 \mu \mathrm{L}, 0.43,1.2 \mathrm{eq})$. The reaction was then allowed to warm to room temperature and was stirred for $4 \mathrm{~h}$. Once reaction was determined to be complete via thin layer chromatographic analysis, the reaction was quenched with saturated aqueous ammonium chloride. The mixture was extracted with ethyl acetate (x3), then dried over anhydrous sodium sulfate, filtered, and concentrated under rotary evanporation. The resulting residue was purified by silica gel chromatography (ethyl acetate/hexanes) to afford 1,3,5-trimethylindole as a colorless oil (56 mg, 22\%). ${ }^{13}$

\footnotetext{
${ }^{12}$ Kimura, M.; Futamata, M.; Mukai, K.; Tamaru, Y. J. Am. Chem. Soc. 2005, 127, 4592-4593.

${ }^{13}$ Repka, L. M.; Ni, J.; Reisman, S. E. J Am. Chem. Soc. 2010, 132, 14418-14420.
} 


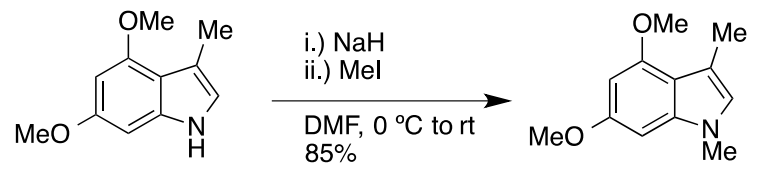

Following general procedure B as described above, a mixture of 3-methyl-4,6-dimethoxyindole (300 mg, $1.57 \mathrm{mmol}, 1$ eq.), $\mathrm{NaH}(113 \mathrm{mg}, 4.71 \mathrm{mmol}, 3 \mathrm{eq})$, and MeI (147 $\mu \mathrm{L}, 2.35 \mathrm{mmol}, 1.5 \mathrm{eq})$ in dry DMF (3.5 mL) afforded 4,5-dimethoxy-1,3-dimethylindole as a colorless oil (272 mg, $85 \%) .{ }^{14}$

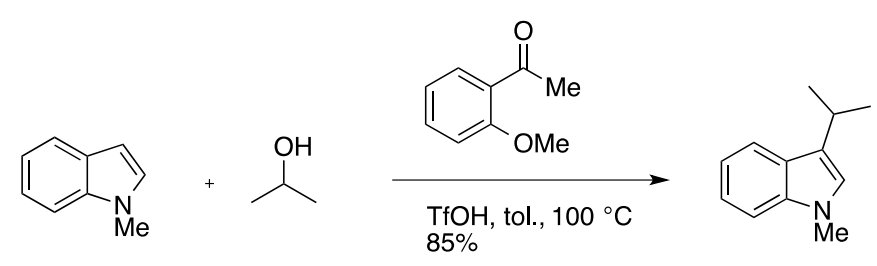

To a round bottom flask containing 1-methylindole $(125 \mu \mathrm{L}, 1 \mathrm{mmol}, 1 \mathrm{eq})$ in toluene $(5 \mathrm{~mL})$, isopropanol ( $150 \mu \mathrm{L}, 2 \mathrm{mmol}, 2 \mathrm{eq})$ and 2-methoxyacetophenone (10 $\mu \mathrm{L}, 0.05 \mathrm{mmol}, 0.05 \mathrm{eq})$ was added, followed by triflic acid $(9 \mu \mathrm{L}, 0.1 \mathrm{mmol}, 0.1 \mathrm{eq})$. The reaction was then heated to $100^{\circ} \mathrm{C}$ and stirred for 2 hours. Once the reaction was determined to be complete via thin layer chromatographic analysis, the reaction mixture was then warmed to room temperature and concentrated under vacuum. The resulting residue was purified by silica gel chromatography (ethyl acetate/hexanes) to afford 1-methyl-3isopropylindole as a colorless oil $(147 \mathrm{mg}, 85 \%) .{ }^{15}$

\section{c. General Procedure $\mathrm{C}$ for $(3+2)$ Annulation reaction}

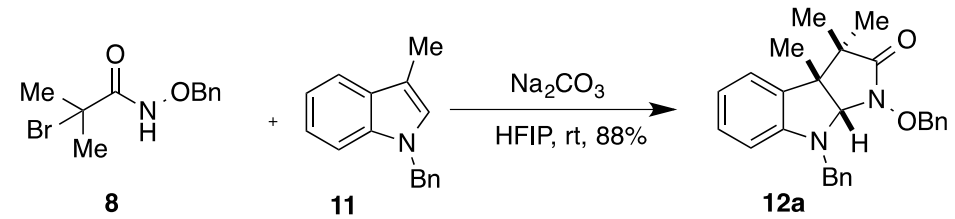

To a solution of indole 11 (37 mg, $0.25 \mathrm{mmol}, 1$ eq.) in HFIP ( $1 \mathrm{~mL}), \alpha$-haloamide 8 (137 mg, $0.5 \mathrm{mmol}$, 2 eq.) and $\mathrm{Na}_{2} \mathrm{CO}_{3}$ (106 mg, $1 \mathrm{mmol}, 4$ eq.) was added. The reaction was stirred at room temperature. Once the reaction was complete as judged by thin layer chromatography analysis, the reaction mixture was diluted with ethyl acetate, filtered through celite and washed with ethyl acetate. The filtrate was then concentrated under rotary evaporation. The resulting residue was purified by silica gel chromatography (ethyl acetate/hexane) to afford indoline 12a as a colorless oil ( $84 \mathrm{mg}, 88 \%)$.

${ }^{1} \mathbf{H}$ NMR $\left(600 \mathrm{MHz}, \mathrm{CD}_{2} \mathrm{Cl}_{2}\right): \delta$, ppm 7.27-7.15 (10H, m), $6.93(2 \mathrm{H}, \mathrm{ddd}, \mathrm{J}=7.2,7.2,14.8 \mathrm{~Hz}), 6.61$ $(1 \mathrm{H}, \mathrm{t}, \mathrm{J}=7.5 \mathrm{~Hz}), 6.25(1 \mathrm{H}, \mathrm{d}, \mathrm{J}=7.9 \mathrm{~Hz}), 5.24(2 \mathrm{H}, \mathrm{d}, \mathrm{J}=8.2 \mathrm{~Hz}), 4.98(1 \mathrm{H}, \mathrm{d}, \mathrm{J}=10.9 \mathrm{~Hz}), 4.82(1 \mathrm{H}$, $\mathrm{d}, \mathrm{J}=10.0 \mathrm{~Hz}), 4.54(2 \mathrm{H}, \mathrm{d}, \mathrm{J}=17.3 \mathrm{~Hz}), 4.37(1 \mathrm{H}, \mathrm{d}, \mathrm{J}=17.3 \mathrm{~Hz}), 1.13(3 \mathrm{H}, \mathrm{s}), 1.10(3 \mathrm{H}, \mathrm{s}), 0.95(3 \mathrm{H}$, s).

${ }^{13}$ C NMR $\left(150 \mathrm{MHz}, \mathrm{CD}_{2} \mathrm{Cl}_{2}\right): 174.1,149.7,138.3,135.1,132.3,129.6,128.8,128.6,128.5,128.3$, 127.2, 127.1, 124.3, 118.2, 107.4, 86.0, 76.7, 53.8, 51.5, 43.5, 24.1, 21.7, 21.6.

IR (film, cm ${ }^{-1}$ ), 3396, 2973, 1698, 1605, 1486, 1285, 744, 699.

HRMS (ESI) calcd. for $\mathrm{C}_{27} \mathrm{H}_{28} \mathrm{~N}_{2} \mathrm{O}_{2}\left(\mathrm{~m} / z \mathrm{M}+\mathrm{H}^{+}\right)$: 413.2229, found: 413.2224.

\footnotetext{
${ }^{14}$ Keawin, T.; Rajviroongit, S.; Black, D. S. Tetrahedron 2006, 61, 863-861.

15 Han, X.; Wu, J. Angew. Chem. Int. Ed. 2013, 52, 4637-4640.
} 


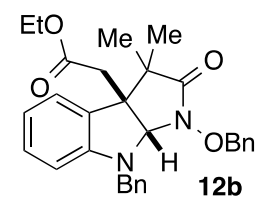

By following the general procedure $\mathrm{C}$ described above, a mixture of N-benzyl-3-ethyl acetate (73 $\mathrm{mg}$, $0.25 \mathrm{mmol}), \alpha$-haloamide 8 (137 mg, $0.5 \mathrm{mmol})$, and $\mathrm{Na}_{2} \mathrm{CO}_{3}(106 \mathrm{mg}, 1 \mathrm{mmol})$ in HFIP (1 mL) afforded indoline 12b as a colorless oil (78 $\mathrm{mg}, 65 \%)$.

${ }^{1} \mathbf{H}$ NMR $\left(600 \mathrm{MHz}, \mathrm{CD}_{2} \mathrm{Cl}_{2}\right): \delta$, ppm 7.46-7.35 $(10 \mathrm{H}, \mathrm{m}), 7.32(1 \mathrm{H}, \mathrm{dd}, \mathrm{J}=7.1,7.1 \mathrm{~Hz}), 7.07(1 \mathrm{H}$, ddd, J = 7.9, 7.9, $1.1 \mathrm{~Hz}), 7.03(1 \mathrm{H}, \mathrm{d}, \mathrm{J}=6.8 \mathrm{~Hz}), 6.73(1 \mathrm{H}, \mathrm{dd}, \mathrm{J}=7.4,7.4 \mathrm{~Hz}), 6.34(1 \mathrm{H}, \mathrm{d}, \mathrm{J}=8.0$ $\mathrm{Hz}), 5.26(1 \mathrm{H}, \mathrm{s}), 5.13(1 \mathrm{H}, \mathrm{d}, \mathrm{J}=12.6 \mathrm{~Hz}), 4.92(1 \mathrm{H}, \mathrm{d}, \mathrm{J}=11.4 \mathrm{~Hz}), 4.66(1 \mathrm{H}, \mathrm{d}, \mathrm{J}=16.7 \mathrm{~Hz}), 4.50$ $(1 \mathrm{H}, \mathrm{d}, \mathrm{J}=16.7 \mathrm{~Hz}), 3.93(2 \mathrm{H}, \mathrm{q}, \mathrm{J}=7.1 \mathrm{~Hz}), 2.87(1 \mathrm{H}, \mathrm{d}, \mathrm{J}=15.3 \mathrm{~Hz}), 2.50(1 \mathrm{H}, \mathrm{d}, \mathrm{J}=15.5 \mathrm{~Hz}), 1.26$ $(3 \mathrm{H}, \mathrm{s}), 1.07(6 \mathrm{H}, \mathrm{t}, \mathrm{J}=8.7 \mathrm{~Hz})$.

${ }^{13}$ C NMR $\left(150 \mathrm{MHz}, \mathrm{CD}_{2} \mathrm{Cl}_{2}\right): 173.8,170.2,151.1,138.5,135.0,129.6,128.9,128.8,128.7,128.5$, $128.4,127.2,127.0,124.3,118.0,107.3,84.6,76.6,60.4,52.7,44.0,39.0,25.4,23.7,21.3,13.7$.

IR (film, cm $\mathrm{cm}^{-1}$ ): 3437, 3061, 2979, 1712, 1605, 1488, 1186, 746, 700.

HRMS (ESI) calcd. for $\mathrm{C}_{30} \mathrm{H}_{32} \mathrm{~N}_{2} \mathrm{O}_{4}\left(\mathrm{~m} / z \mathrm{M}+\mathrm{H}^{+}\right)$: 485.2440, found: 485.2444.

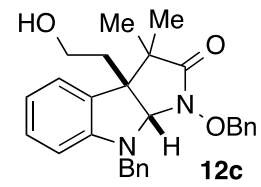

By following the general procedure $\mathrm{C}$ described above, a mixture of $\mathrm{N}$-benzyl-tryptophol (63 $\mathrm{mg}, 0.25$ mmol), $\alpha$-haloamide 8 (137 mg, $0.5 \mathrm{mmol})$, and $\mathrm{Na}_{2} \mathrm{CO}_{3}(106 \mathrm{mg}, 1 \mathrm{mmol})$ in $\mathrm{HFIP}(1 \mathrm{~mL})$ afforded indoline 12c as a white solid (70 $\mathrm{mg}, 63 \%)$.

${ }^{1} \mathbf{H}$ NMR $\left(600 \mathrm{MHz}, \mathrm{CD}_{2} \mathrm{Cl}_{2}\right): \delta, \mathrm{ppm} 7.29-7.17(10 \mathrm{H}, \mathrm{m}), 6.98(1 \mathrm{H}, \mathrm{ddd}, \mathrm{J}=7.7,7.7,1.0 \mathrm{~Hz}), 6.89$ $(1 \mathrm{H}, \mathrm{d}, \mathrm{J}=7.1 \mathrm{~Hz}), 6.62(1 \mathrm{H}, \mathrm{dd}, \mathrm{J}=7.4,7.4 \mathrm{~Hz}), 6.32(1 \mathrm{H}, \mathrm{d}, \mathrm{J}=7.9 \mathrm{~Hz}), 4.98(1 \mathrm{H}, \mathrm{d}, \mathrm{J}=10.5 \mathrm{~Hz})$, $4.81(1 \mathrm{H}, \mathrm{d}, \mathrm{J}=10.5 \mathrm{~Hz}), 4.80(1 \mathrm{H}, \mathrm{s}), 4.53(1 \mathrm{H}, \mathrm{d}, \mathrm{J}=15.5 \mathrm{~Hz}), 4.37(1 \mathrm{H}, \mathrm{d}, \mathrm{J}=16.0 \mathrm{~Hz}), 3.15(1 \mathrm{H}$, ddd, $\mathrm{J}=5.8,10.8,13.2 \mathrm{~Hz}), 3.07(1 \mathrm{H}, \mathrm{ddd}, \mathrm{J}=11.9,11.9,6.9 \mathrm{~Hz}), 1.93(1 \mathrm{H}$, ddd, $\mathrm{J}=5.8,8.0,14.1 \mathrm{~Hz})$, $1.63(1 \mathrm{H}, \mathrm{ddd}, \mathrm{J}=6.6,7.7,14.3 \mathrm{~Hz}), 1.12(3 \mathrm{H}, \mathrm{s}), 0.95(3 \mathrm{H}, \mathrm{s})$.

${ }^{13} \mathrm{C}$ NMR (150 MHz, $\left.\mathrm{CD}_{2} \mathrm{Cl}_{2}\right): 173.8,150.4,138.0,135.1,129.7,129.0,128.8,128.6,128.5,128.4$, $127.5,127.2,125.0,118.2,107.7,82.9,76.5,59.4,54.0,51.4,44.1,36.3,24.1,21.9$.

IR (film, $\mathrm{cm}^{-1}$ ): 3419, 3062, 2976, 2359, 1686, 1485, 1265, 1037, 734.

MELTING POINT: $48.1-48.4^{\circ} \mathrm{C}$

HRMS (ESI) calcd. for $\mathrm{C}_{28} \mathrm{H}_{30} \mathrm{~N}_{2} \mathrm{O}_{3}\left(\mathrm{~m} / z \mathrm{M}+\mathrm{H}^{+}\right)$: 443.2335, found: 443.2338 .

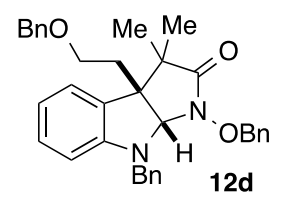

By following the general procedure $\mathrm{C}$ described above, a mixture of N-benzyl-O-benzyl tryptophol (85 $\mathrm{mg}, 0.25 \mathrm{mmol}), \alpha$-haloamide 8 (137 mg, $0.5 \mathrm{mmol})$, and $\mathrm{Na}_{2} \mathrm{CO}_{3}(106 \mathrm{mg}, 1 \mathrm{mmol})$ in HFIP (1 mL) afforded indoline $\mathbf{1 2 d}$ as a colorless oil $(83 \mathrm{mg}, 62 \%)$. 
${ }^{1} \mathbf{H}$ NMR $\left(600 \mathrm{MHz}, \mathrm{CD}_{2} \mathrm{Cl}_{2}\right): \delta$, ppm 7.26-7.14 $(13 \mathrm{H}, \mathrm{m}), 7.09(2 \mathrm{H}, \mathrm{dd}, \mathrm{J}=2.1,7.5 \mathrm{~Hz}), 6.98(1 \mathrm{H}$, ddd, J = 7.7, 7.7, $1.5 \mathrm{~Hz}), 6.86(1 \mathrm{H}, \mathrm{dd}, \mathrm{J}=1.2,7.3 \mathrm{~Hz}), 6.62(1 \mathrm{H}, \mathrm{ddd}, \mathrm{J}=7.5,7.5,1.0 \mathrm{~Hz}), 6.28(1 \mathrm{H}, \mathrm{d}$, $\mathrm{J}=7.6 \mathrm{~Hz}), 4.93(1 \mathrm{H}, \mathrm{d}, \mathrm{J}=11.7 \mathrm{~Hz}), 4.83(1 \mathrm{H}, \mathrm{s}), 4.79(1 \mathrm{H}, \mathrm{d}, \mathrm{J}=9.9 \mathrm{~Hz}), 4.46(1 \mathrm{H}, \mathrm{d}, \mathrm{J}=14.4 \mathrm{~Hz})$, $4.27(1 \mathrm{H}, \mathrm{d}, \mathrm{J}=14.4 \mathrm{~Hz}), 4.12(2 \mathrm{H}, \mathrm{dd}, \mathrm{J}=9.5,25.7 \mathrm{~Hz}), 3.03(1 \mathrm{H}, \mathrm{ddd}, \mathrm{J}=5.3,7.9,9.2 \mathrm{~Hz}), 2.95(1 \mathrm{H}$, ddd, $\mathrm{J}=6.9,7.9,9.2 \mathrm{~Hz}), 2.02(1 \mathrm{H}, \mathrm{ddd}, \mathrm{J}=5.1,7.9,14.0 \mathrm{~Hz}), 1.73(1 \mathrm{H}$, ddd, $\mathrm{J}=6.2,7.7,14.0 \mathrm{~Hz})$, $1.11(3 \mathrm{H}, \mathrm{s}), 0.91(3 \mathrm{H}, \mathrm{s})$.

${ }^{13} \mathrm{C}$ NMR $\left(150 \mathrm{MHz}, \mathrm{CD}_{2} \mathrm{Cl}_{2}\right): 173.9,150.7,138.4,138.2,135.1,129.6,128.9,128.8,128.6,128.5$, $128.4,128.2$, 127.5, 127.4, 127.1, 124.8, 118.2, 118.0, 107.5, 83.3, 76.5, 72.9, 67.4, 54.1, 51.7, 44.2, 33.6, 24.6, 21.6.

IR (film, $\mathrm{cm}^{-1}$ ): 3418, 2985, 2252, 1696, 1643, 1265, 907, 732, 649.

HRMS (ESI) calcd. for $\mathrm{C}_{35} \mathrm{H}_{36} \mathrm{~N}_{2} \mathrm{O}_{3}\left(\mathrm{~m} / z \mathrm{M}+\mathrm{H}^{+}\right)$: 533.2804, found: 533.2808.

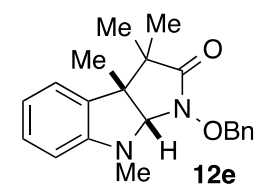

By following the general procedure $\mathrm{C}$ described above, a mixture of $\mathrm{N}$-benzyl-3-methylindole (50 $\mathrm{mg}$, $0.225 \mathrm{mmol}$ ), $\alpha$-haloamide 8 (123 mg, $0.452 \mathrm{mmol}$ ), and $\mathrm{Na}_{2} \mathrm{CO}_{3}(95.7 \mathrm{mg}, 0.903 \mathrm{mmol})$ in HFIP afforded indoline 12e (118 $\mathrm{mg}, 93 \%)$ as a colorless oil.

${ }^{1} \mathbf{H}$ NMR $\left(600 \mathrm{MHz}, \mathrm{CD}_{2} \mathrm{Cl}_{2}\right): \delta$, ppm $7.52(2 \mathrm{H}, \mathrm{dd}, \mathrm{J}=1.8,7.6 \mathrm{~Hz}), 7.49-7.40(3 \mathrm{H}, \mathrm{m}), 7.16(1 \mathrm{H}, \mathrm{ddd}$, $\mathrm{J}=7.7,7.7,1.0 \mathrm{~Hz}), 7.01(1 \mathrm{H}, \mathrm{d}, \mathrm{J}=7.6 \mathrm{~Hz}), 6.74(1 \mathrm{H}, \mathrm{t}, \mathrm{J}=7.7 \mathrm{~Hz}), 6.49(1 \mathrm{H}, \mathrm{d}, \mathrm{J}=7.9 \mathrm{~Hz}), 5.15(1 \mathrm{H}$, d, J = 10.7 Hz), $5.03(1 \mathrm{H}, \mathrm{d}, \mathrm{J}=11.5 \mathrm{~Hz}), 4.41(1 \mathrm{H}, \mathrm{s}), 2.98(3 \mathrm{H}, \mathrm{s}), 1.26(3 \mathrm{H}, \mathrm{s}), 1.23(3 \mathrm{H}, \mathrm{s}), 1.00(3 \mathrm{H}$, s).

${ }^{13}$ C NMR (150 MHz, $\left.\mathrm{CD}_{2} \mathrm{Cl}_{2}\right): 174.1,150.7,135.3,132.2,129.6,128.9,128.5,128.4,124.0,118.1$, $107.1,88.0,76.8,51.3,43.5,34.6,24.1,21.9,21.4$.

IR (film, $\mathrm{cm}^{-1}$ ): 3434, 2972, 1707, 1605, 1488, 1282, 1089, 1020, 746.

HRMS (ESI) calcd. for $\mathrm{C}_{21} \mathrm{H}_{24} \mathrm{~N}_{2} \mathrm{O}_{2}\left(\mathrm{~m} / z \mathrm{M}+\mathrm{H}^{+}\right)$: 337.1916, found: 337.1911 .

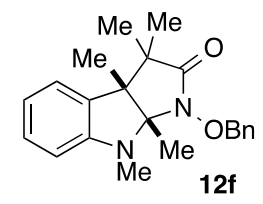

By following the general procedure $\mathrm{C}$ described above, a mixture of 1,2,3-trimethylindole (40 $\mathrm{mg}, 0.25$ mmol), $\alpha$-haloamide 8 (137 mg, $0.5 \mathrm{mmol})$, and $\mathrm{Na}_{2} \mathrm{CO}_{3}(106 \mathrm{mg}, 1 \mathrm{mmol})$ in $\mathrm{HFIP}(1 \mathrm{~mL})$ afforded indoline $\mathbf{1 2 f}$ as a colorless oil (38 $\mathrm{mg}, 42 \%)$.

${ }^{1} \mathbf{H}$ NMR $\left(600 \mathrm{MHz}, \mathrm{CD}_{2} \mathrm{Cl}_{2}\right): \delta$, ppm $7.37(2 \mathrm{H}, \mathrm{dd}, \mathrm{J}=1.7,7.4 \mathrm{~Hz}), 7.32-7.27(3 \mathrm{H}, \mathrm{m}), 7.02(1 \mathrm{H}, \mathrm{ddd}$, $\mathrm{J}=7.8,7.8,1.4 \mathrm{~Hz}), 6.95(1 \mathrm{H}, \mathrm{dd}, \mathrm{J}=1.2,7.4 \mathrm{~Hz}), 6.59(1 \mathrm{H}, \mathrm{dd}, \mathrm{J}=7.6,7.6 \mathrm{~Hz}), 6.29(1 \mathrm{H}, \mathrm{d}, \mathrm{J}=8.1$

$\mathrm{Hz}), 4.98(1 \mathrm{H}, \mathrm{d}, \mathrm{J}=9.7 \mathrm{~Hz}), 4.83(1 \mathrm{H}, \mathrm{d}, \mathrm{J}=9.8 \mathrm{~Hz}), 2.83(3 \mathrm{H}, \mathrm{s}), 1.38(3 \mathrm{H}, \mathrm{s}), 1.16(3 \mathrm{H}, \mathrm{s}), 1.13(3 \mathrm{H}$, $\mathrm{s}), 1.05(3 \mathrm{H}, \mathrm{s})$.

${ }^{13} \mathbf{C}$ NMR $\left(150 \mathrm{MHz}, \mathrm{CD}_{2} \mathrm{Cl}_{2}\right): 173.7,149.0,135.2,132.2,129.3,128.6,128.4,128.3,125.0,117.3$, $105.9,87.7,78.2,77.4,43.2,29.3,23.9,22.6,18.3,16.0$.

IR (film, $\mathrm{cm}^{-1}$ ): 3419, 3053, 2986, 1698, 1604, 1491, 1265, 1104, 1039, 737, 702.

HRMS (ESI) calcd. for $\mathrm{C}_{22} \mathrm{H}_{26} \mathrm{~N}_{2} \mathrm{O}_{2}\left(\mathrm{~m} / z \mathrm{M}+\mathrm{H}^{+}\right)$: 351.2073, found: 351.2071 . 


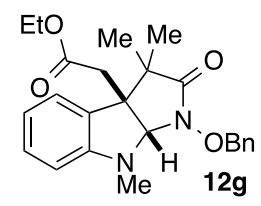

By following the general procedure $\mathrm{C}$ described above, a mixture of $\mathrm{N}$-methyl-3-indole-ethyl acetate (54 $\mathrm{mg}, 0.25 \mathrm{mmol}), \alpha$-haloamide 8 (137 mg, $0.5 \mathrm{mmol})$, and $\mathrm{Na}_{2} \mathrm{CO}_{3}(106 \mathrm{mg}, 1 \mathrm{mmol})$ in HFIP (1 mL) afforded indoline $\mathbf{1 2 g}$ as a colorless oil $(69 \mathrm{mg}, 68 \%)$.

${ }^{1} \mathbf{H}$ NMR (600 MHz, $\left.\mathrm{CD}_{2} \mathrm{Cl}_{2}\right): \delta$, ppm $7.40(2 \mathrm{H}, \mathrm{dd}, \mathrm{J}=1.5,7.2 \mathrm{~Hz}), 7.34-7.27(3 \mathrm{H}, \mathrm{m}), 7.04$ (1H, ddd, $\mathrm{J}=7.8,7.8,1.3 \mathrm{~Hz}), 6.86(1 \mathrm{H}, \mathrm{d}, \mathrm{J}=7.6 \mathrm{~Hz}), 6.59(1 \mathrm{H}, \mathrm{dd}, \mathrm{J}=7.6,7.6 \mathrm{~Hz}), 6.34(1 \mathrm{H}, \mathrm{d}, \mathrm{J}=8.3 \mathrm{~Hz})$, $5.06(1 \mathrm{H}, \mathrm{d}, \mathrm{J}=9.7 \mathrm{~Hz}), 4.93(2 \mathrm{H}, \mathrm{d}, \mathrm{J}=11.9 \mathrm{~Hz}), 4.92(2 \mathrm{H}, \mathrm{s}), 3.77(2 \mathrm{H}, \mathrm{dq}, \mathrm{J}=2.2,7.2 \mathrm{~Hz}), 2.87(3 \mathrm{H}$, s), $2.71(1 \mathrm{H}, \mathrm{d}, \mathrm{J}=15.2 \mathrm{~Hz}), 2.36(1 \mathrm{H}, \mathrm{d}, \mathrm{J}=15.2 \mathrm{~Hz}), 1.11(3 \mathrm{H}, \mathrm{s}), 0.91(3 \mathrm{H}, \mathrm{t}, \mathrm{J}=7.0 \mathrm{~Hz}), 0.89(3 \mathrm{H}$, s).

${ }^{13}$ C NMR $\left(150 \mathrm{MHz}, \mathrm{CD}_{2} \mathrm{Cl}_{2}\right): 173.4,170.1,151.6,135.2,129.6,128.9,128.8,128.6,128.5,124.5$, $117.7,107.1,85.6,76.4,60.3,53.1,43.8,39.2,34.9,29.6,24.9,21.6,13.6$.

IR (film, cm ${ }^{-1}$ ): 3427, 2979, 2878, 1708, 1644, 1607, 1490, 1283, 1184, 1028, 736, 700.

HRMS (ESI) calcd. for $\mathrm{C}_{24} \mathrm{H}_{28} \mathrm{~N}_{2} \mathrm{O}_{4}\left(\mathrm{~m} / z \mathrm{M}+\mathrm{H}^{+}\right)$: 409.2127, found: 409.2130 .

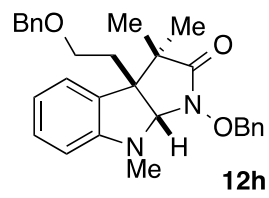

By following the general procedure $\mathrm{C}$ described above, a mixture of O-benzyl-N-methyltryptophol (66 $\mathrm{mg}, 0.25 \mathrm{mmol}), \alpha$-haloamide 8 (137 mg, $0.5 \mathrm{mmol})$, and $\mathrm{Na}_{2} \mathrm{CO}_{3}(106 \mathrm{mg}, 1 \mathrm{mmol})$ in HFIP (1 mL) afforded indoline $\mathbf{1 2 h}$ as a colorless oil ( $86 \mathrm{mg}, 76 \%$ ).

${ }^{1} \mathbf{H}$ NMR $\left(600 \mathrm{MHz}, \mathrm{CD}_{2} \mathrm{Cl}_{2}\right): \delta$, ppm $7.35(2 \mathrm{H}, \mathrm{q}, \mathrm{J}=3.3 \mathrm{~Hz}), 7.32-7.25(3 \mathrm{H}, \mathrm{m}), 7.21(2 \mathrm{H}, \mathrm{dd}, \mathrm{J}=$ 7.2, 7.2 Hz), 7.16 (1H, dd, J = 7.4, 7.4 Hz), 7.09 (2H, d, J = 7.4 Hz), $7.04(1 \mathrm{H}, \mathrm{ddd}, \mathrm{J}=7.6,7.6,1.2 \mathrm{~Hz})$, $6.83(1 \mathrm{H}, \mathrm{d}, \mathrm{J}=7.4 \mathrm{~Hz}), 6.60(1 \mathrm{H}, \mathrm{dd}, \mathrm{J}=7.4,7.4 \mathrm{~Hz}), 6.34(1 \mathrm{H}, \mathrm{d}, \mathrm{J}=7.8 \mathrm{~Hz}), 4.97(1 \mathrm{H}, \mathrm{d}, \mathrm{J}=10.9$ $\mathrm{Hz}), 4.86(1 \mathrm{H}, \mathrm{d}, \mathrm{J}=12.0 \mathrm{~Hz}), 4.67(1 \mathrm{H}, \mathrm{s}), 4.15(2 \mathrm{H}, \mathrm{dd}, \mathrm{J}=12.6,18.3 \mathrm{~Hz}), 3.06(1 \mathrm{H}, \mathrm{ddd}, \mathrm{J}=5.3,7.2$, $9.4 \mathrm{~Hz}), 2.98(1 \mathrm{H}, \mathrm{ddd}, \mathrm{J}=7.1,7.1,9.3 \mathrm{~Hz}), 2.79(3 \mathrm{H}, \mathrm{s}), 1.95(1 \mathrm{H}, \mathrm{ddd}, \mathrm{J}=5.1,6.9,14.0 \mathrm{~Hz}), 1.79(1 \mathrm{H}$, ddd, $\mathrm{J}=7.1,7.1,14.3 \mathrm{~Hz}), 1.11(3 \mathrm{H}, \mathrm{s}), 0.91(3 \mathrm{H}, \mathrm{s})$.

${ }^{13} \mathrm{C}$ NMR $\left(150 \mathrm{MHz}, \mathrm{CD}_{2} \mathrm{Cl}_{2}\right)$ : 173.7, 151.3, 138.4, 135.3, 129.6, 129.0, 128.9, 128.6, 128.5, 128.2, $127.6,127.5,127.4,124.9,117.8,107.0,85.1,76.7,72.9,67.3,54.2,44.0,34.3,33.6,24.1,22.2$.

IR (film, $\mathrm{cm}^{-1}$ ): 3440, 3060, 3030, 2973, 2871, 1706, 1604, 1490, 1282, 1097, 1026, 744, 699.

HRMS (ESI) calcd. for $\mathrm{C}_{29} \mathrm{H}_{32} \mathrm{~N}_{2} \mathrm{O}_{3}\left(\mathrm{~m} / z \mathrm{M}+\mathrm{H}^{+}\right)$: 457.2491, found: 457.2497.

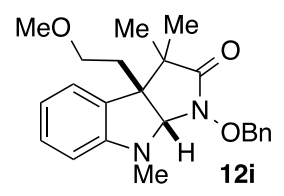

By following the general procedure $\mathrm{C}$ described above, a mixture of $\mathrm{N}$, O-dimethyl-tryptophol (47 $\mathrm{mg}$, $0.25 \mathrm{mmol}), \alpha$-haloamide 8 (137 mg, $0.5 \mathrm{mmol})$, and $\mathrm{Na}_{2} \mathrm{CO}_{3}(106 \mathrm{mg}, 1 \mathrm{mmol})$ in HFIP (1 mL) afforded indoline 12i as a colorless oil (62 $\mathrm{mg}, 66 \%)$.

${ }^{1} \mathbf{H}$ NMR $\left(600 \mathrm{MHz}, \mathrm{CD}_{2} \mathrm{Cl}_{2}\right): \delta$, ppm $7.39(2 \mathrm{H}, \mathrm{dd}, \mathrm{J}=1.7,7.1 \mathrm{~Hz}), 7.34-7.28(3 \mathrm{H}, \mathrm{m}), 7.05(1 \mathrm{H}$, ddd, $\mathrm{J}=7.6,7.6,1.1 \mathrm{~Hz}), 6.85(1 \mathrm{H}, \mathrm{dd}, \mathrm{J}=1.0,7.3 \mathrm{~Hz}), 6.61(1 \mathrm{H}, \mathrm{ddd}, \mathrm{J}=7.4,7.4,0.7 \mathrm{~Hz}), 6.35(1 \mathrm{H}, \mathrm{d}, \mathrm{J}=$ 
$7.3 \mathrm{~Hz}), 5.01(1 \mathrm{H}, \mathrm{d}, \mathrm{J}=11.1 \mathrm{~Hz}), 4.89(1 \mathrm{H}, \mathrm{d}, \mathrm{J}=10.0 \mathrm{~Hz}), 4.61,(1 \mathrm{H}, \mathrm{s}), 3.01(3 \mathrm{H}, \mathrm{s}), 2.91(1 \mathrm{H}, \mathrm{ddd}, \mathrm{J}$ $=5.2,7.7,9.5 \mathrm{~Hz}), 2.86(3 \mathrm{H}, \mathrm{s}), 2.81(1 \mathrm{H}, \mathrm{ddd}, \mathrm{J}=7.1,7.1,9.4 \mathrm{~Hz}), 1.89(1 \mathrm{H}, \mathrm{ddd}, \mathrm{J}=5.9,8.3,13.3$ $\mathrm{Hz}), 1.75(1 \mathrm{H}, \mathrm{ddd}, \mathrm{J}=7.2,7.2,14.1 \mathrm{~Hz}), 1.13(3 \mathrm{H}, \mathrm{s}), 0.94(3 \mathrm{H}, \mathrm{s})$.

${ }^{13}$ C NMR (150 MHz, $\left.\mathrm{CD}_{2} \mathrm{Cl}_{2}\right)$ : 173.6, 151.2, 135.3, 129.6, 129.1, 128.8, 128.6, 128.5, 125.0, 117.8, 107.0, 85.0, 76.6, 69.4, 58.2, 54.2, 43.9, 34.4, 33.6, 23.9, 22.2.

IR (film, $\mathrm{cm}^{-1}$ ): 3438, 3053, 2975, 2927, 2877, 2832, 2810, 1703, 1604, 1490, 1282, 1115, 1026, 745, 700 .

HRMS (ESI) calcd. for $\mathrm{C}_{23} \mathrm{H}_{28} \mathrm{~N}_{2} \mathrm{O}_{3}\left(\mathrm{~m} / z \mathrm{M}+\mathrm{H}^{+}\right)$: 381.2178 , found: 381.2177 .

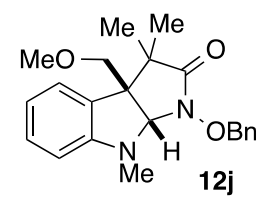

By following the general procedure $\mathrm{C}$ described above, a mixture of $\mathrm{N}$-methyl-tryptophol (44 $\mathrm{mg}, 0.25$ mmol), $\alpha$-haloamide 8 (137 mg, $0.5 \mathrm{mmol})$, and $\mathrm{Na}_{2} \mathrm{CO}_{3}(106 \mathrm{mg}, 1 \mathrm{mmol})$ in $\mathrm{HFIP}(1 \mathrm{~mL})$ afforded indoline $\mathbf{1 2} \mathbf{j}$ as a white solid $(57 \mathrm{mg}, 63 \%)$.

${ }^{1} \mathbf{H}$ NMR $\left(600 \mathrm{MHz}, \mathrm{CD}_{2} \mathrm{Cl}_{2}\right): \delta$, ppm $7.41(2 \mathrm{H}, \mathrm{dd}, \mathrm{J}=1.3,8.4 \mathrm{~Hz}), 7.35-7.28(3 \mathrm{H}, \mathrm{m}), 7.06(1 \mathrm{H}$, ddd, $\mathrm{J}=7.7,7.7,1.1 \mathrm{~Hz}), 6.86(1 \mathrm{H}, \mathrm{dd}, \mathrm{J}=0.8,7.3 \mathrm{~Hz}), 6.61(1 \mathrm{H}, \mathrm{ddd}, \mathrm{J}=7.4,7.4,0.7 \mathrm{~Hz}), 6.37(1 \mathrm{H}, \mathrm{d}, \mathrm{J}=$ $8.1 \mathrm{~Hz}), 5.02(1 \mathrm{H}, \mathrm{d}, \mathrm{J}=11.4 \mathrm{~Hz}), 4.95(1 \mathrm{H}, \mathrm{d}, \mathrm{J}=10.5 \mathrm{~Hz}), 4.70(1 \mathrm{H}, \mathrm{s}), 3.42(2 \mathrm{H}, \mathrm{dd}, \mathrm{J}=9.1,24.1$ $\mathrm{Hz}), 3.17(3 \mathrm{H}, \mathrm{s}), 2.83(3 \mathrm{H}, \mathrm{s}), 1.15(3 \mathrm{H}, \mathrm{s}), 0.80(3 \mathrm{H}, \mathrm{s})$.

${ }^{13}$ C NMR $\left(150 \mathrm{MHz}, \mathrm{CD}_{2} \mathrm{Cl}_{2}\right): 174.5,152.0,135.5,129.5,128.8,128.7,128.4,128.1,123.7,118.0$, $107.3,84.5,76.8,74.4,58.7,55.5,42.7,35.0,29.6,25.6,19.2$.

IR (film, $\mathrm{cm}^{-1}$ ): 3425, 2924, 2095, 1642, 1488, 1278, 1095, 1024, 745, 698.

MELTING POINT: $59.9-60.8^{\circ} \mathrm{C}$

HRMS (ESI) calcd. for $\mathrm{C}_{22} \mathrm{H}_{26} \mathrm{~N}_{2} \mathrm{O}_{3}\left(\mathrm{~m} / z \mathrm{M}+\mathrm{H}^{+}\right)$: 367.2022 , found: 367.2015 .

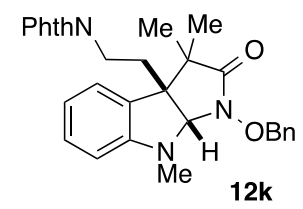

By following the general procedure $\mathrm{C}$ described above, a mixture of $\mathrm{N}$-methyl-N'-phthalimidetryptamine (76 mg, $0.25 \mathrm{mmol}$ ), $\alpha$-haloamide 8 (137 mg, $0.5 \mathrm{mmol}$ ), and $\mathrm{Na}_{2} \mathrm{CO}_{3}(106 \mathrm{mg}, 1 \mathrm{mmol})$ in HFIP (1 mL) afforded indoline $\mathbf{1 2 k}$ as a yellow solid $(66 \mathrm{mg}, 54 \%)$.

${ }^{1} \mathbf{H}$ NMR $\left(600 \mathrm{MHz}, \mathrm{CD}_{2} \mathrm{Cl}_{2}\right): \delta$, ppm 7.64-7.61 (2H, m), 7.61-7.57 (2H, m), $7.43(2 \mathrm{H}, \mathrm{d}, \mathrm{J}=7.5 \mathrm{~Hz})$, $7.36-7.29(3 \mathrm{H}, \mathrm{m}), 6.91(1 \mathrm{H}, \mathrm{ddd}, \mathrm{J}=7.7,7.7,0.9 \mathrm{~Hz}), 6.88(1 \mathrm{H}, \mathrm{d}, \mathrm{J}=7.6 \mathrm{~Hz}), 6.30(1 \mathrm{H}, \mathrm{d}, \mathrm{J}=7.2$ $\mathrm{Hz}), 6.47(1 \mathrm{H}, \mathrm{dd}, \mathrm{J}=7.9,7.9 \mathrm{~Hz}), 5.06(1 \mathrm{H}, \mathrm{d}, \mathrm{J}=10.1 \mathrm{~Hz}), 4.93(1 \mathrm{H}, \mathrm{d}, \mathrm{J}=10.6 \mathrm{~Hz}), 4.79(1 \mathrm{H}, \mathrm{s})$, $3.29(1 \mathrm{H}, \mathrm{ddd}, \mathrm{J}=4.1,10.3,14.2 \mathrm{~Hz}), 3.13(1 \mathrm{H}, \mathrm{ddd}, \mathrm{J}=6.9,9.3,13.9 \mathrm{~Hz}), 2.91(3 \mathrm{H}, \mathrm{s}), 2.19(1 \mathrm{H}$, ddd, $\mathrm{J}=7.3,10.2,13.8 \mathrm{~Hz}), 1.66(1 \mathrm{H}, \mathrm{ddd}, \mathrm{J}=4.1,9.6,13.6 \mathrm{~Hz}), 1.10(3 \mathrm{H}, \mathrm{s}), 0.88(3 \mathrm{H}, \mathrm{s})$.

${ }^{13} \mathbf{C}$ NMR $\left(150 \mathrm{MHz}, \mathrm{CD}_{2} \mathrm{Cl}_{2}\right): 173.6,167.7,151.1,135.2,133.7,132.0,129.6,128.9,128.7,128.6$, $128.1,124.5,122.8,118.0,107.2,84.3,76.8,54.0,44.2,34.3,34.2,31.6,24.1,21.5$. IR (film, $\mathrm{cm}^{-1}$ ): 3424, 3058, 2974, 1770, 1709, 1489, 1401, 1372, 1286, 1034, 732.

MELTING POINT: $95.2-96.7^{\circ} \mathrm{C}$

HRMS (ESI) calcd. for $\mathrm{C}_{30} \mathrm{H}_{29} \mathrm{~N}_{3} \mathrm{O}_{4}\left(\mathrm{~m} / z \mathrm{M}+\mathrm{H}^{+}\right)$: 496.2236 , found: 496.2241 . 


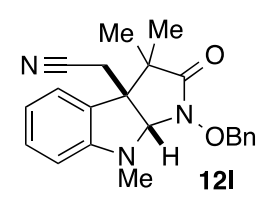

By following the general procedure $\mathrm{C}$ described above, a mixture of $\mathrm{N}$-methylindole-3-acetonitrile (43 $\mathrm{mg}, 0.25 \mathrm{mmol})$, $\alpha$-haloamide 8 (137 mg, $0.5 \mathrm{mmol})$, and $\mathrm{Na}_{2} \mathrm{CO}_{3}(106 \mathrm{mg}, 1 \mathrm{mmol})$ in HFIP (1 mL) afforded indoline $\mathbf{1 2 l}$ as a white solid $(67 \mathrm{mg}, 75 \%)$.

${ }^{1} \mathbf{H}$ NMR $\left(500 \mathrm{MHz}, \mathrm{CD}_{2} \mathrm{Cl}_{2}\right): \delta$, ppm $7.52(2 \mathrm{H}, \mathrm{ddd}, \mathrm{J}=3.3,3.3,3.3 \mathrm{~Hz}), 7.47(3 \mathrm{H}, \mathrm{ddd}, \mathrm{J}=4.2,4.2$, $1.7 \mathrm{~Hz}), 7.27(1 \mathrm{H}, \mathrm{ddd}, \mathrm{J}=7.6,7.6,1.3 \mathrm{~Hz}), 7.19(1 \mathrm{H}, \mathrm{dd}, \mathrm{J}=0.9,7.5 \mathrm{~Hz}), 6.83(1 \mathrm{H}, \mathrm{ddd}, \mathrm{J}=7.6,7.6$, $1.1 \mathrm{~Hz}), 6.57(1 \mathrm{H}, \mathrm{d}, \mathrm{J}=7.7 \mathrm{~Hz}), 5.19(1 \mathrm{H}, \mathrm{d}, \mathrm{J}=11.7 \mathrm{~Hz}), 5.04(1 \mathrm{H}, \mathrm{d}, \mathrm{J}=11.7 \mathrm{~Hz}), 4.64(1 \mathrm{H}, \mathrm{s}), 3.04$ $(3 \mathrm{H}, \mathrm{s}), 2.78(1 \mathrm{H}, \mathrm{d}, \mathrm{J}=18.4 \mathrm{~Hz}), 2.50(1 \mathrm{H}, \mathrm{d}, \mathrm{J}=18.4 \mathrm{~Hz}), 1.31(3 \mathrm{H}, \mathrm{s}), 1.17(3 \mathrm{H}, \mathrm{s})$.

${ }^{13}$ C NMR $\left(150 \mathrm{MHz}, \mathrm{CD}_{2} \mathrm{Cl}_{2}\right)$ : 172.4, 150.4, 134.8, 129.8, 129.7, 129.1, 128.6, 127.4, 124.7, 118.5, $116.8,107.7,85.0,76.9,52.7,43.4,34.2,24.0,23.9,21.8$.

IR (film, $\mathrm{cm}^{-1}$ ): 3435, 3056, 2978, 2879, 2252, 1705, 1606, 1490, 1283, 1030, 987, 735, 700.

MELTING POINT: $94.8-95.2^{\circ} \mathrm{C}$

HRMS (ESI) calcd. for $\mathrm{C}_{22} \mathrm{H}_{23} \mathrm{~N}_{3} \mathrm{O}_{2}\left(\mathrm{~m} / z \mathrm{M}+\mathrm{H}^{+}\right): 362.1869$, found: 362.1870 .

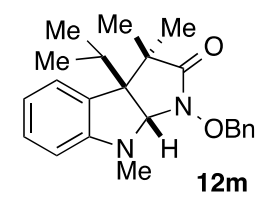

By following the general procedure $\mathrm{C}$ described above, a mixture of $\mathrm{N}$-methyl-3-isopropylindole (39 $\mathrm{mg}$, $0.25 \mathrm{mmol}), \alpha$-haloamide 8 (137 mg, $0.5 \mathrm{mmol})$, and $\mathrm{Na}_{2} \mathrm{CO}_{3}(106 \mathrm{mg}, 1 \mathrm{mmol})$ in HFIP $(1 \mathrm{~mL})$ afforded indoline $12 \mathrm{~m}$ as a colorless oil (45 $\mathrm{mg}, 54 \%)$.

${ }^{1} \mathbf{H}$ NMR $\left(600 \mathrm{MHz}, \mathrm{CD}_{2} \mathrm{Cl}_{2}\right): \delta$, ppm $7.39(2 \mathrm{H}, \mathrm{dd}, \mathrm{J}=2.3,7.3 \mathrm{~Hz}), 7.35-7.29(3 \mathrm{H}, \mathrm{m}), 7.06(1 \mathrm{H}$, ddd, $\mathrm{J}=7.7,7.7,1.2 \mathrm{~Hz}), 6.91(1 \mathrm{H}, \mathrm{d}, \mathrm{J}=7.5 \mathrm{~Hz}), 6.61(1 \mathrm{H}, \mathrm{dd}, \mathrm{J}=7.5,7.5 \mathrm{~Hz}), 6.35(1 \mathrm{H}, \mathrm{d}, \mathrm{J}=8.6 \mathrm{~Hz})$, $5.02(1 \mathrm{H}, \mathrm{d}, \mathrm{J}=10.0 \mathrm{~Hz}), 4.88(1 \mathrm{H}, \mathrm{d}, \mathrm{J}=10.0 \mathrm{~Hz}), 4.54(1 \mathrm{H}, \mathrm{s}), 2.87(3 \mathrm{H}, \mathrm{s}), 2.21-2.10(1 \mathrm{H}, \mathrm{m}), 1.20$ $(4 \mathrm{H}, \mathrm{s}), 1.03(3 \mathrm{H}, \mathrm{s}), 0.74(3 \mathrm{H}, \mathrm{d}, \mathrm{J}=8.5 \mathrm{~Hz}), 0.58(3 \mathrm{H}, \mathrm{d}, \mathrm{J}=6.3 \mathrm{~Hz})$.

${ }^{13}$ C NMR $\left(150 \mathrm{MHz}, \mathrm{CD}_{2} \mathrm{Cl}_{2}\right): 174.6,151.3,135.3,129.4,128.7,128.5,128.4,128.1,125.9,117.4$, $106.8,83.4,76.8,59.2,44.0,34.0,30.9,25.5,21.3,18.8,17.5$.

IR (film, $\mathrm{cm}^{-1}$ ): 3461, 2972, 2872, 1706, 1605, 1488, 1281, 1105, 1020, 745, 700.

HRMS (ESI) calcd. for $\mathrm{C}_{21} \mathrm{H}_{24} \mathrm{~N}_{2} \mathrm{O}_{2}\left(\mathrm{~m} / z \mathrm{M}+\mathrm{H}^{+}\right)$: 337.1916, found: 337.1916 .

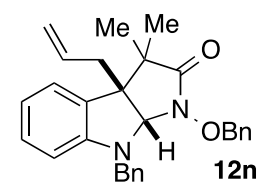

By following the general procedure $\mathrm{C}$ described above, a mixture of $\mathrm{N}$-benzyl-3-allylindole $(61 \mathrm{mg}, 0.25$ mmol), $\alpha$-haloamide 8 (137 mg, $0.5 \mathrm{mmol})$, and $\mathrm{Na}_{2} \mathrm{CO}_{3}(106 \mathrm{mg}, 1 \mathrm{mmol})$ in HFIP (1 mL) afforded indoline 12n as a colorless oil (27 mg, 25\%).

${ }^{1} \mathbf{H}$ NMR $\left(600 \mathrm{MHz}, \mathrm{CDCl}_{3}\right): \delta$, ppm 7.36-7.24 (10H, m), $7.04(1 \mathrm{H}, \mathrm{ddd}, \mathbf{J}=7.6,7.6,0.8 \mathrm{~Hz}), 6.99$ $(1 \mathrm{H}, \mathrm{dd}, \mathrm{J}=0.9,7.3 \mathrm{~Hz}), 6.71(1 \mathrm{H}, \mathrm{ddd}, \mathrm{J}=7.4,7.4,1.0 \mathrm{~Hz}), 6.35(1 \mathrm{H}, \mathrm{d}, \mathrm{J}=8.3 \mathrm{~Hz}), 5.29-5.21(1 \mathrm{H}$, m), $5.06(1 \mathrm{H}, \mathrm{d}, \mathrm{J}=11.4 \mathrm{~Hz}), 4.86(3 \mathrm{H}, \mathrm{ddd}, \mathrm{J}=13.0,13.0,3.4 \mathrm{~Hz}), 4.81(1 \mathrm{H}, \mathrm{s}), 4.60(1 \mathrm{H}, \mathrm{d}, \mathrm{J}=19.4$ 
$\mathrm{Hz}), 4.43(1 \mathrm{H}, \mathrm{d}, \mathrm{J}=14.8 \mathrm{~Hz}), 2.63(1 \mathrm{H}, \mathrm{dd}, \mathrm{J}=5.1,14.3 \mathrm{~Hz}), 2.17(1 \mathrm{H}, \mathrm{q}, \mathrm{J}=7.6 \mathrm{~Hz}), 1.25(3 \mathrm{H}, \mathrm{s})$, $1.02(3 \mathrm{H}, \mathrm{s})$.

${ }^{13}$ C NMR $\left(150 \mathrm{MHz}, \mathrm{CD}_{2} \mathrm{Cl}_{2}\right): 174.2,151.0,138.1,135.2,134.2,129.6,128.9,128.6,128.5,127.5$, $127.1,124.7,118.6,118.1,107.4,82.9,76.6,55.0,51.7,44.0,38.7,25.1,21.2$.

IR (film, $\mathrm{cm}^{-1}$ ): 3433, 3062, 2936, 2869, 1706, 1606, 1266, 1027, 949, 736, 699.

HRMS (ESI) calcd. for $\mathrm{C}_{29} \mathrm{H}_{30} \mathrm{~N}_{2} \mathrm{O}_{2}\left(\mathrm{~m} / z \mathrm{M}+\mathrm{H}^{+}\right)$: 439.2386, found: 439.2383 .

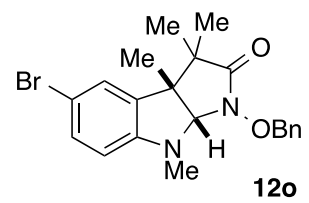

By following the general procedure $\mathrm{C}$ described above, a mixture of $\mathrm{N}$-methyl-3-methyl-5-bromoindole (56 mg, $0.25 \mathrm{mmol}$ ), $\alpha$-haloamide 8 (137 mg, $0.5 \mathrm{mmol})$, and $\mathrm{Na}_{2} \mathrm{CO}_{3}(106 \mathrm{mg}, 1 \mathrm{mmol})$ in $\mathrm{HFIP}(1 \mathrm{~mL})$ afforded indoline $\mathbf{1 2 0}$ as a yellow oil (77 $\mathrm{mg}, 72 \%)$.

${ }^{1} \mathbf{H}$ NMR $\left(600 \mathrm{MHz}, \mathrm{CD}_{2} \mathrm{Cl}_{2}\right): \delta$, ppm $7.47(2 \mathrm{H}, \mathrm{dd}, \mathrm{J}=1.9,7.3 \mathrm{~Hz}), 7.40-7.38(3 \mathrm{H}, \mathrm{m}), 7.21(1 \mathrm{H}, \mathrm{dd}, \mathbf{J}$ $=2.1,8.9 \mathrm{~Hz}), 7.05(1 \mathrm{H}, \mathrm{d}, \mathrm{J}=1.8 \mathrm{~Hz}), 6.30(1 \mathrm{H}, \mathrm{d}, \mathrm{J}=8.4 \mathrm{~Hz}), 5.01(1 \mathrm{H}, \mathrm{d}, \mathrm{J}=10.5 \mathrm{~Hz}), 4.97(1 \mathrm{H}, \mathrm{d}, \mathrm{J}$ $=10.5 \mathrm{~Hz}), 4.35(1 \mathrm{H}, \mathrm{s}), 2.92(3 \mathrm{H}, \mathrm{s}), 1.18(3 \mathrm{H}, \mathrm{s}), 1.17(3 \mathrm{H}, \mathrm{s}), 0.98(3 \mathrm{H}, \mathrm{s})$.

${ }^{13}$ C NMR $\left(150 \mathrm{MHz}, \mathrm{CD}_{2} \mathrm{Cl}_{2}\right): 173.8,149.7,135.2,134.6,131.1,129.7$ 129.0, 128.6, 127.0, 109.6, $108.5,87.9,76.9,51.4,43.4,34.4,24.1,21.7,21.5$.

IR (film, $\mathrm{cm}^{-1}$ ): 3429, 3054, 2978, 2937, 2875, 2304, 1706, 1600, 1487, 1266, 1018, 808, 734, 701. HRMS (ESI) calcd. for $\mathrm{C}_{21} \mathrm{H}_{23} \mathrm{~N}_{2} \mathrm{O}_{2} \mathrm{Br}\left(\mathrm{m} / z \mathrm{M}+\mathrm{H}^{+}\right)$: 415.1021, found: 415.1022 .

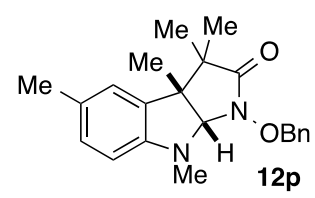

By following the general procedure $\mathrm{C}$ described above, a mixture of 1,3,5-trimethylindole ( $39 \mathrm{mg}, 0.25$ mmol), $\alpha$-haloamide 8 (137 mg, $0.5 \mathrm{mmol})$, and $\mathrm{Na}_{2} \mathrm{CO}_{3}(106 \mathrm{mg}, 1 \mathrm{mmol})$ in HFIP (1 mL) afforded indoline 12p as a colorless oil (56 $\mathrm{mg}, 65 \%)$.

${ }^{1} \mathbf{H}$ NMR $\left(600 \mathrm{MHz}, \mathrm{CD}_{2} \mathrm{Cl}_{2}\right): \delta$, ppm $7.52(2 \mathrm{H}, \mathrm{dd}, \mathrm{J}=1.7,7.6 \mathrm{~Hz}), 7.47-7.41(3 \mathrm{H}, \mathrm{m}), 6.98(1 \mathrm{H}, \mathrm{d}, \mathrm{J}$ $=8.2 \mathrm{~Hz}), 6.83(1 \mathrm{H}, \mathrm{s}), 6.40(1 \mathrm{H}, \mathrm{d}, \mathrm{J}=8.2 \mathrm{~Hz}), 5.16(1 \mathrm{H}, \mathrm{d}, \mathrm{J}=11.3 \mathrm{~Hz}), 5.04(1 \mathrm{H}, \mathrm{d}, \mathrm{J}=10.0 \mathrm{~Hz})$, $4.37(1 \mathrm{H}, \mathrm{s}), 2.96(3 \mathrm{H}, \mathrm{s}), 2.28(3 \mathrm{H}, \mathrm{s}), 1.26(3 \mathrm{H}, \mathrm{s}), 1.22(3 \mathrm{H}, \mathrm{s}), 1.00(3 \mathrm{H}, \mathrm{s})$.

${ }^{13}$ C NMR (150 MHz, $\left.\mathrm{CD}_{2} \mathrm{Cl}_{2}\right)$ : 174.2, 148.8, 135.3, 132.3, 129.6, 128.8, 128.6, 128.5, 127.6, 124.7, 107.1, 88.6, 76.8, 51.3, 43.4, 35.1, 24.3, 22.0, 21.2, 20.5.

IR (film, $\mathrm{cm}^{-1}$ ): 3461, 2973, 2933, 2689, 2300, 1706, 1616, 1498, 1455, 1373, 1278, 1093, 1019, 807, 749, 701.

HRMS (ESI) calcd. for $\mathrm{C}_{22} \mathrm{H}_{26} \mathrm{~N}_{2} \mathrm{O}_{2}\left(\mathrm{~m} / z \mathrm{M}+\mathrm{H}^{+}\right)$: 351.2073, found: 351.2075 .

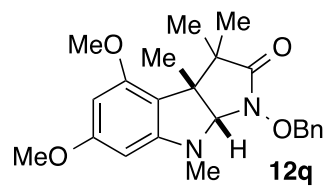

By following the general procedure $\mathrm{C}$ described above, a mixture of $\mathrm{N}$-methyl-4, 6-dimethoxyindole (51 $\mathrm{mg}, 0.25 \mathrm{mmol}), \alpha$-haloamide 8 (137 mg, $0.5 \mathrm{mmol})$, and $\mathrm{Na}_{2} \mathrm{CO}_{3}(106 \mathrm{mg}, 1 \mathrm{mmol})$ in HFIP (1 mL) afforded indoline 12q as a white solid $(65 \mathrm{mg}, 72 \%)$. 
${ }^{1} \mathbf{H}$ NMR $\left(600 \mathrm{MHz}, \mathrm{CD}_{2} \mathrm{Cl}_{2}\right): \delta$, ppm 7.41-7.38 (2H, m), 7.34-7.29 (3H, m), $5.79(1 \mathrm{H}, \mathrm{d}, \mathrm{J}=2.3 \mathrm{~Hz})$, $5.61(1 \mathrm{H}, \mathrm{d}, \mathrm{J}=2.0 \mathrm{~Hz}), 5.03(1 \mathrm{H}, \mathrm{d}, \mathrm{J}=10.5 \mathrm{~Hz}), 4.94(1 \mathrm{H}, \mathrm{d}, \mathrm{J}=10.4 \mathrm{~Hz}), 4.14(1 \mathrm{H}, \mathrm{s}), 3.66(6 \mathrm{H}, \mathrm{s})$, $2.78(3 \mathrm{H}, \mathrm{s}), 1.22(3 \mathrm{H}, \mathrm{s}), 1.13(3 \mathrm{H}, \mathrm{s}), 0.74(3 \mathrm{H}, \mathrm{s})$.

${ }^{13} \mathrm{C} \mathrm{NMR}\left(150 \mathrm{MHz}, \mathrm{CD}_{2} \mathrm{Cl}_{2}\right): 175.1,162.1,157.2,153.7,135.2,129.6,128.8,128.5,128.3,126.8$, $110.2,89.8,88.8,87.0,76.9,55.2,54.9,50.8,43.8,35.3,25.3,22.3,20.1$.

IR (film, cm $\mathrm{cm}^{-1}$ ): 3447, 2971, 2938, 1707, 1609, 1455, 1208, 1149, 1058, 804, 746, 700.

MELTING POINT: $109.3-110.4^{\circ} \mathrm{C}$

HRMS (ESI) calcd. for $\mathrm{C}_{23} \mathrm{H}_{28} \mathrm{~N}_{2} \mathrm{O}_{4}\left(\mathrm{~m} / z \mathrm{M}+\mathrm{H}^{+}\right)$: 397.2127, found: 397.2129.

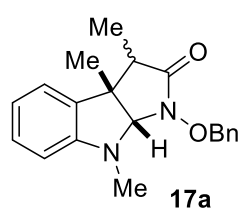

By following the general procedure $\mathrm{C}$ described above but at $50{ }^{\circ} \mathrm{C}$, a mixture of 1,3-dimethylindole (108 mg, $0.75 \mathrm{mmol}$ ), $\alpha$-haloamide $16 a\left(213 \mathrm{mg}, 1.25 \mathrm{mmol}\right.$ ), and $\mathrm{Na}_{2} \mathrm{CO}_{3}(265 \mathrm{mg}, 2.50 \mathrm{mmol}$ ) in HFIP (3 mL) afforded an inseparable 1:1 diastereomeric mixture of indoline 17a as a colorless oil (131 $\mathrm{mg}, 54 \%)$.

As a 1:1 diastereomer mixture:

${ }^{1} \mathbf{H}$ NMR $\left(600 \mathrm{MHz}, \mathrm{CD}_{2} \mathrm{Cl}_{2}\right): \delta$, ppm $7.38(4 \mathrm{H}, \mathrm{d}, \mathrm{J}=8.0 \mathrm{~Hz}), 7.35-7.27(5 \mathrm{H}, \mathrm{m}), 7.23-7.14(1 \mathrm{H}, \mathrm{m})$, $7.05-7.00(2 \mathrm{H}, \mathrm{m}), 6.88(2 \mathrm{H}, \mathrm{d}, \mathrm{J}=7.5 \mathrm{~Hz}), 6.61(2 \mathrm{H}, \mathrm{dt}, \mathrm{J}=4.3,7.2 \mathrm{~Hz}), 6.34(2 \mathrm{H}, \mathrm{t}, \mathrm{J}=7.7 \mathrm{~Hz}), 5.00$ $(2 \mathrm{H}, \mathrm{dd}, \mathrm{J}=10.5,15.7 \mathrm{~Hz}), 4.87(2 \mathrm{H}, \mathrm{dd}, \mathrm{J}=3.8,11.2 \mathrm{~Hz}), 4.33(1 \mathrm{H}, \mathrm{s}), 4.26(1 \mathrm{H}, \mathrm{s}), 2.87(3 \mathrm{H}, \mathrm{s}), 2.86$ $(3 \mathrm{H}, \mathrm{s}), 2.48(1 \mathrm{H}, \mathrm{q}, \mathrm{J}=7.1 \mathrm{~Hz}), 2.33(1 \mathrm{H}, \mathrm{q}, \mathrm{J}=7.7 \mathrm{~Hz}), 1.24(3 \mathrm{H}, \mathrm{s}), 1.14(3 \mathrm{H}, \mathrm{d}, \mathrm{J}=9.8 \mathrm{~Hz}), 1.08$ $(3 \mathrm{H}, \mathrm{s}), 0.95(3 \mathrm{H}, \mathrm{d}, \mathrm{J}=8.3 \mathrm{~Hz})$.

${ }^{13}$ C NMR (150 MHz, $\left.\mathrm{CD}_{2} \mathrm{Cl}_{2}\right)$ : ppm, 171.6, 171.5, 150.5, 149.4, 135.6, 135.3, 130.8, 129.7, 129.6, 128.94, 128.90, 128.6, 128.5, 128.4, 124.6, 121.8, 118.3, 117.8, 107.1, 107.0, 88.1, 87.9, 76.9, 48.0, $47.2,44.2,43.3,34.3,34.1,26.6,19.6,13.7,11.4$.

IR (film, cm-1): 3460, 3032, 2973, 2301, 1708, 1607, 1490, 1276, 1058, 954, 747, 710.

HRMS (ESI) calcd. for $\mathrm{C}_{20} \mathrm{H}_{22} \mathrm{~N}_{2} \mathrm{O}_{2}\left(\mathrm{~m} / z \mathrm{M}+\mathrm{H}^{+}\right)$: 323.1760, found: 323.1758 .

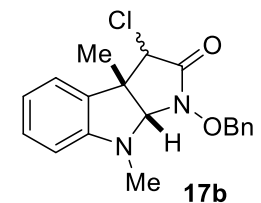

By following the general procedure $\mathrm{C}$ described above, a mixture of 1,3-dimethylindole (36 $\mathrm{mg}, 0.25$ mmol), $\alpha$-haloamide $\mathbf{1 6 b}$ (137 mg, $0.5 \mathrm{mmol})$, and $\mathrm{Na}_{2} \mathrm{CO}_{3}(106 \mathrm{mg}, 1 \mathrm{mmol})$ in HFIP (1 mL) afforded a 1:1 diastereomeric mixture indoline $\mathbf{1 7 b}$ as a colorless oil (36 $\mathrm{mg}, 42 \%)$. Purification by silica gel flash chromatography provided an analytically pure sample of the following diastereomer.

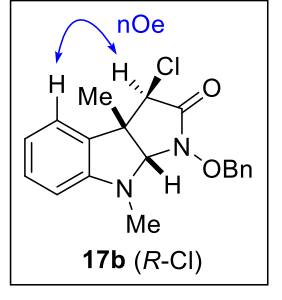

${ }^{1} \mathbf{H}$ NMR $\left(600 \mathrm{MHz}, \mathrm{CD}_{2} \mathrm{Cl}_{2}\right): \delta$, ppm 7.54-7.50 (2H, m), 7.50-7.45 (3H, m), 7.21 $(1 \mathrm{H}, \mathrm{ddd}, \mathrm{J}=7.9,7.9,1.2 \mathrm{~Hz}), 7.08(1 \mathrm{H}, \mathrm{d}, \mathrm{J}=7.5 \mathrm{~Hz}), 6.79(1 \mathrm{H}, \mathrm{dd}, \mathrm{J}=7.3,7.3$ $\mathrm{Hz}), 6.50(1 \mathrm{H}, \mathrm{d}, \mathrm{J}=7.7 \mathrm{~Hz}), 5.12(1 \mathrm{H}, \mathrm{d}, \mathrm{J}=10.9 \mathrm{~Hz}), 4.99(1 \mathrm{H}, \mathrm{d}, \mathrm{J}=14.5 \mathrm{~Hz})$, $4.56(1 \mathrm{H}, \mathrm{s}), 4.43(1 \mathrm{H}, \mathrm{s}), 2.98(3 \mathrm{H}, \mathrm{s}), 1.34(3 \mathrm{H}, \mathrm{s})$.

${ }^{13}$ C NMR $\left(150 \mathrm{MHz}, \mathrm{CD}_{2} \mathrm{Cl}_{2}\right): 134.7,130.0,129.7,129.2,128.6,122.7,118.7$, $107.4,86.0,76.7,60.5,33.2,29.3,20.8,13.8$. IR (film, $\mathrm{cm}^{-1}$ ): 3418, 2926, 2851, 2196, 1721, 1640, 1609, 993, 711, 678.

HRMS (ESI) calcd. for $\mathrm{C}_{19} \mathrm{H}_{19} \mathrm{ClN}_{2} \mathrm{O}_{4}\left(\mathrm{~m} / z \mathrm{M}+\mathrm{H}^{+}\right)$: 343.1213 , found: 343.1210 . 


\section{d. General Procedure $\mathrm{D}$ for $\mathrm{N}-\mathrm{O}$ bond cleavage reaction ${ }^{16}$}
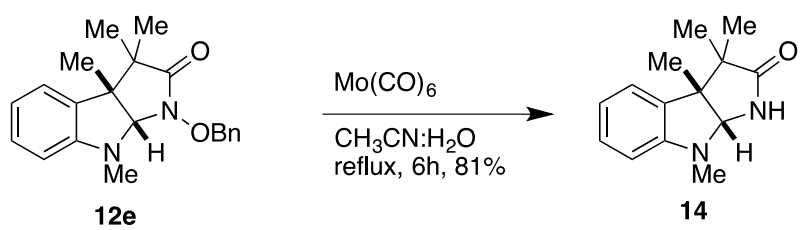

To a solution of indoline $12 \mathrm{e}(100 \mathrm{mg}, 0.2 \mathrm{mmol}, 1 \mathrm{eq})$ in acetonitrile/water $(9: 1,2 \mathrm{~mL}), \mathrm{Mo}(\mathrm{CO})_{6}(95$ $\mathrm{mg}, 0.357 \mathrm{mmol}, 1.2 \mathrm{eq}$ ) was added. The reaction was stirred at reflux for $6 \mathrm{~h}$. Once the reaction was determined to be complete via thin layer chromatographic analysis, the reaction mixture was cooled to room temperature. The mixture was the filtered through celite and washed with ethyl acetate. Then, filtrate was concentrated under rotary evaporation, and the resulting residue was purified by silica gel chromatography (ethyl acetate/hexane) to afford 14 as a white solid (41 $\mathrm{mg}, 81 \%$ ).

${ }^{1} \mathbf{H}$ NMR $\left(600 \mathrm{MHz}, \mathrm{CD}_{2} \mathrm{Cl}_{2}\right): \delta$, ppm $7.11(1 \mathrm{H}, \mathrm{s}), 7.03(1 \mathrm{H}, \mathrm{ddd}, \mathrm{J}=7.9,7.9,1.4 \mathrm{~Hz}), 6.93(1 \mathrm{H}, \mathrm{dd}, \mathrm{J}$ $=1.0,7.2 \mathrm{~Hz}), 6.62(1 \mathrm{H}, \mathrm{ddd}, \mathrm{J}=7.3,7.3,0.7 \mathrm{~Hz}), 6.36(1 \mathrm{H}, \mathrm{d}, \mathrm{J}=7.4 \mathrm{~Hz}), 4.55(1 \mathrm{H}, \mathrm{d}, \mathrm{J}=0.9 \mathrm{~Hz})$, $2.72(3 \mathrm{H}, \mathrm{s}), 1.26(3 \mathrm{H}, \mathrm{s}), 1.10(3 \mathrm{H}, \mathrm{s}), 0.93(3 \mathrm{H}, \mathrm{s})$.

${ }^{13}$ C NMR $\left(150 \mathrm{MHz}, \mathrm{CD}_{2} \mathrm{Cl}_{2}\right): 182.8,150.5,132.8,128.2,124.2,117.9,107.0,84.4,54.2,45.5,32.3$, 23.3, 21.7, 21.2.

IR (film, cm ${ }^{-1}$ ): 3422, 2970, 2919, 2869, 2824, 2359, 1694, 1487, 1288, 1024, 740.

MELTING POINT: $147.2-148.0^{\circ} \mathrm{C}$

HRMS (ESI) calcd. for $\mathrm{C}_{14} \mathrm{H}_{18} \mathrm{~N}_{2} \mathrm{O}\left(\mathrm{m} / z \mathrm{M}+\mathrm{H}^{+}\right)$: 231.1497, found: 231.1502 .

\section{e. General Procedure E for Protodehalogenation reaction ${ }^{17}$}

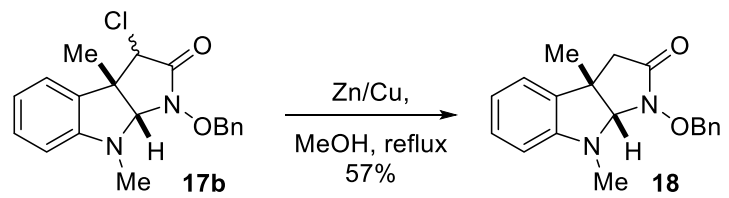

To a round bottom flask containing indoline $\mathbf{1 7 b}$ (36 mg, $0.105 \mathrm{mmol})$ in $\mathrm{MeOH}(3 \mathrm{~mL}), \mathrm{Zn} / \mathrm{Cu}$ couple (290 $\mathrm{mg}, 2.22 \mathrm{mmol}$ ) was added. The reaction mixture was stirred at reflux for $7 \mathrm{~h}$. Once the reaction was determined to be complete via thin layer chromatographic analysis, the reaction mixture was filtered and repeatedly washed with $\mathrm{MeOH}$. The filtrate was then diluted with water, extracted with $\mathrm{CH}_{2} \mathrm{Cl}_{2}$ (x3), dried over anhydrous sodium sulfate, and concentrated under rotary evaporation. The resulting residue was purified by silica gel chromatography (ethyl acetate/hexanes) to afford 18 (18 $\mathrm{mg}, 57 \%$ ).

${ }^{1} \mathbf{H}$ NMR $\left(600 \mathrm{MHz}, \mathrm{CD}_{2} \mathrm{Cl}_{2}\right): \delta$, ppm $7.37(2 \mathrm{H}, \mathrm{dd}, \mathrm{J}=2.1,7.7 \mathrm{~Hz}), 7.34-7.28(3 \mathrm{H}, \mathrm{m}), 7.03(1 \mathrm{H}$, ddd, $\mathrm{J}=7.7,7.7,1.2 \mathrm{~Hz}), 6.93(1 \mathrm{H}, \mathrm{dd}, \mathrm{J}=0.7,7.3 \mathrm{~Hz}), 6.63(1 \mathrm{H}, \mathrm{ddd}, \mathrm{J}=7.5,7.5,0.8 \mathrm{~Hz}), 6.35(1 \mathrm{H}, \mathrm{d}, \mathrm{J}=$ $8.1 \mathrm{~Hz}), 5.02(1 \mathrm{H}, \mathrm{d}, \mathrm{J}=10.8 \mathrm{~Hz}), 4.83(1 \mathrm{H}, \mathrm{d}, \mathrm{J}=10.4 \mathrm{~Hz}), 4.43(1 \mathrm{H}, \mathrm{s}), 2.91(3 \mathrm{H}, \mathrm{s}), 2.57(1 \mathrm{H}, \mathrm{d}, \mathrm{J}=$ $17.3 \mathrm{~Hz}), 2.40(1 \mathrm{H}, \mathrm{d}, \mathrm{J}=16.2 \mathrm{~Hz}), 1.25(3 \mathrm{H}, \mathrm{s})$.

${ }^{13}$ C NMR $\left(150 \mathrm{MHz}, \mathrm{CD}_{2} \mathrm{Cl}_{2}\right): 168.5,149.0,135.2,135.1,129.6,128.8,128.6,128.5,122.1,118.3$, $106.9,88.0,76.8,43.7,41.4,33.6,25.9$.

IR (film, cm $\mathrm{cm}^{-1}$ ): 3430, 3055, 2926, 2097, 1641, 1492, 1265, 736, 702.

HRMS (ESI) calcd. for $\mathrm{C}_{19} \mathrm{H}_{20} \mathrm{~N}_{2} \mathrm{O}_{2}\left(\mathrm{~m} / z \mathrm{M}+\mathrm{H}^{+}\right)$: 309.1603, found: 309.1602 .

\footnotetext{
${ }^{16}$ Zhao, G-L.; Lin, S.; Korotvicka, A.; Deiana, L.; Kullberg, M.; Cordova, Adv. Synth. Cat. 2010, 352, 2291-2298.

${ }^{17}$ Sondengam, B.L.; Fomum, Z.T.; Charles, G.; Akam, T.M. J. Chem. Soc. Perkin. Trans. I 1983, 1219-1221.
} 


\section{SUPPORTING INFORMATION}

\section{DFT Calculation Details.}

Full-molecule calculations were carried out using the hybrid B3LYP-D3 functional ${ }^{1}$ with the zero-damping, two-body only D3 correction of Grimme et al., ${ }^{2}$ and the 6$311+\mathrm{G}^{*}$ basis, ${ }^{3}$ as implemented in the Jaguar ${ }^{4}$ suite of programs. All computations were carried out using the Poisson-Boltzmann solver ${ }^{5}$ implemented as part of the Jaguar suite, and using an implicit trifluoroethanol solvent model with settings: dielectric constant 26.7; molecular weight $100.04 \mathrm{~g} / \mathrm{mol}$; density $1.39 \mathrm{~g} / \mathrm{mL}$; probe radius $2.43 \AA$. Computed structures were confirmed as stationary points by calculating the vibrational frequencies using second derivative analytic methods, and confirming the absence of imaginary frequencies for minima, and the presence of a single imaginary frequency for transition states. Thermodynamic quantities were calculated assuming an ideal gas, and are zero point energy corrected. Graphical representations of structures were made using the CYLView program. ${ }^{6}$

\section{Material Relevant to all DFT output}

Jaguar version 7.9 , release 23

Copyright Schrodinger, LLC

All Rights Reserved.

J aguar, version 7.9, Schrodinger, LLC, New York, NY, 2011

Non-default options chosen:

SCF calculation type: DFT(b3lyp-d3)

DFT=Becke_3_Parameter/ HF+Slater+Becke88+VWN+LYP (B3LYP)

Solvation energy will be computed using PBF

Vibrational frequencies and related properties computed from analytic second derivatives

Molecular symmetry not used

Energy convergence criterion: $1.00 \mathrm{E}-05$ hartrees

RMS density matrix convergence criterion: $1.00 \mathrm{E}-06$

Highest accuracy cutoffs used in SCF 


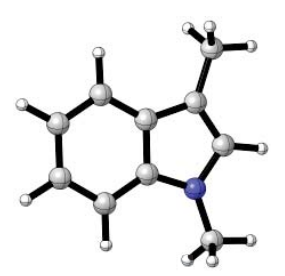

\begin{tabular}{rrrr}
15 & & & \\
$\mathrm{C}$ & -1.21670 & 1.69910 & 0.13790 \\
$\mathrm{C}$ & -2.53040 & 1.80950 & 0.62520 \\
$\mathrm{C}$ & -3.51440 & 0.97370 & 0.10650 \\
$\mathrm{C}$ & -3.21000 & 0.02440 & -0.89320 \\
$\mathrm{C}$ & -1.91850 & -0.10720 & -1.39650 \\
$\mathrm{C}$ & -0.93170 & 0.73840 & -0.87530 \\
$\mathrm{C}$ & 0.01670 & 2.37840 & 0.43800 \\
$\mathrm{C}$ & 0.96840 & 1.82040 & -0.38110 \\
$\mathrm{~N}$ & 0.40780 & 0.83620 & -1.18200 \\
$\mathrm{H}$ & -2.77420 & 2.53540 & 1.39570 \\
$\mathrm{H}$ & -4.53320 & 1.04850 & 0.47420 \\
$\mathrm{H}$ & -3.99850 & -0.61580 & -1.27650 \\
$\mathrm{H}$ & -1.69010 & -0.84040 & -2.16340 \\
$\mathrm{H}$ & 2.02290 & 2.05200 & -0.46630 \\
$\mathrm{C}$ & 1.11780 & -0.00470 & -2.13190 \\
$\mathrm{H}$ & 2.12960 & 0.37940 & -2.26470 \\
$\mathrm{H}$ & 1.17570 & -1.03880 & -1.77610 \\
$\mathrm{H}$ & 0.61310 & 0.00650 & -3.10200 \\
$\mathrm{C}$ & 0.20650 & 3.47820 & 1.43980 \\
$\mathrm{H}$ & 1.24810 & 3.80820 & 1.47430 \\
$\mathrm{H}$ & -0.41240 & 4.35080 & 1.20140 \\
$\mathrm{H}$ & -0.07510 & 3.15600 & 2.44880 \\
& & & \\
\hline & & &
\end{tabular}

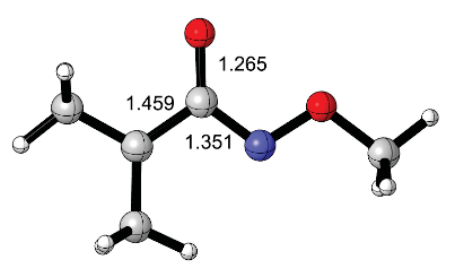

$\begin{array}{crrr}\text { 9-Me } & & & \\ \mathrm{C} & -0.21990 & 5.03400 & -1.17580 \\ \mathrm{~N} & 1.93660 & 4.35980 & -1.52140 \\ \mathrm{C} & -1.33320 & 5.96150 & -1.44060 \\ \mathrm{H} & -1.03140 & 6.97200 & -1.12600 \\ \mathrm{H} & -2.25440 & 5.67380 & -0.93440 \\ \mathrm{C} & 1.03420 & 5.30320 & -1.87030 \\ \mathrm{O} & 1.19040 & 6.27700 & -2.66290 \\ \mathrm{C} & -0.45900 & 3.92310 & -0.22950 \\ \mathrm{H} & -0.80280 & 4.34720 & 0.72700 \\ \mathrm{H} & -1.31130 & 3.32970 & -0.59640 \\ \mathrm{H} & 0.40400 & 3.28460 & -0.07100 \\ \mathrm{H} & -1.49260 & 6.04670 & -2.52380 \\ \mathrm{O} & 3.11850 & 4.56150 & -2.14240 \\ \mathrm{C} & 4.08110 & 3.54840 & -1.76150 \\ \mathrm{H} & 4.99850 & 3.80770 & -2.28920 \\ \mathrm{H} & 4.24200 & 3.57150 & -0.68100 \\ \mathrm{H} & 3.72830 & 2.56170 & -2.07130 \\ & & & \\ ---------------------------------\end{array}$

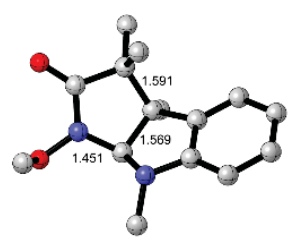

12

C -1.34150

$\begin{array}{lll}-2.60710 & 2.08320 & 0.35320\end{array}$

$\begin{array}{llll}\text { C } & -3.52110 & 1.07030 & 0.02570\end{array}$

$\begin{array}{llll}\text { C } & -3.14600 & 0.05140 & -0.85260\end{array}$

$\begin{array}{llll}\text { C } & -1.86780 & 0.01820 & -1.42340\end{array}$

$\begin{array}{llll}\text { C } & -0.97330 & 1.04150 & -1.10050\end{array}$

$\begin{array}{llll}\text { C } & -0.18230 & 3.01860 & -0.02200\end{array}$

$\begin{array}{llll}\text { C } & 0.96650 & 2.27680 & -0.79190\end{array}$

$\mathrm{N} \quad 0.33190 \quad 1.21720 \quad-1.57790$

$\begin{array}{llll}\mathrm{H} & -2.89170 & 2.86840 & 1.04740\end{array}$

H $\quad-4.51490 \quad 1.07370 \quad 0.46110$

H $\quad-3.85360 \quad-0.73600 \quad-1.09580$

$\begin{array}{llll}\mathrm{H} & -1.59010 & -0.78380 & -2.09860\end{array}$

H $\quad 1.73190 \quad 1.85910 \quad-0.12540$

$\begin{array}{llll}\text { C } & 1.13600 & 0.04690 & -1.92210\end{array}$

$\begin{array}{llll}\mathrm{H} & 2.12870 & 0.36880 & -2.23830\end{array}$

$\mathrm{H} \quad 1.24590 \quad-0.64010 \quad-1.07000$

H $\quad 0.67810 \quad-0.49500 \quad-2.75200$

$\begin{array}{llll}\text { C } & 0.19200 & 3.18540 & 1.45750\end{array}$

$\mathrm{H} \quad 1.10330 \quad 3.77930 \quad 1.57480$

$\begin{array}{llll}H & -0.61020 & 3.67160 & 2.01650\end{array}$

$\begin{array}{llll}\mathrm{H} & 0.36510 & 2.20260 & 1.90290\end{array}$

$\begin{array}{llll}\text { C } & -0.33510 & 4.40250 & -0.79090\end{array}$

N $\quad 1.57400 \quad 3.33390 \quad-1.57950$

C $\quad-1.37960 \quad 4.33220 \quad-1.93050$

$\mathrm{H} \quad-2.38670 \quad 4.26270 \quad-1.51490$

H $\quad-1.21560 \quad 3.46570 \quad-2.57410$

C $\quad 1.02300 \quad 4.56960 \quad-1.48530$

$\begin{array}{llll}0 & 1.49960 & 5.60020 & -1.95260\end{array}$

$\begin{array}{llll}\text { C } & -0.65280 & 5.60870 & 0.09930\end{array}$

$\begin{array}{llll}\mathrm{H} & -0.70640 & 6.50860 & -0.51790\end{array}$

$\begin{array}{llll}\mathrm{H} & -1.62250 & 5.47510 & 0.58740\end{array}$

$\mathrm{H} \quad 0.10340 \quad 5.77650 \quad 0.86780$

$\mathrm{H} \quad-1.31960 \quad 5.23640 \quad-2.54090$

$\begin{array}{llll}0 & 2.81200 & 3.12530 & -2.16920\end{array}$

C $\quad 2.69040 \quad 3.01010 \quad-3.61120$

$\begin{array}{llll}\mathrm{H} & 3.70420 & 2.81460 & -3.96490\end{array}$

$\begin{array}{llll}\mathrm{H} & 2.03020 & 2.18100 & -3.87240\end{array}$

$\begin{array}{llll}\mathrm{H} & 2.31520 & 3.94460 & -4.03610\end{array}$

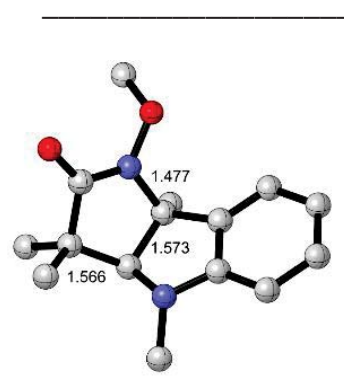
21
$\begin{array}{llll}\text { C } & -0.34610 & 1.89330 & 0.37610 \\ \text { C } & -0.19900 & 1.75280 & 1.74710\end{array}$ 


$\begin{array}{rrrr}\text { C } & -1.22140 & 1.13310 & 2.48080 \\ \mathrm{C} & -2.35800 & 0.65530 & 1.82140 \\ \mathrm{C} & -2.51190 & 0.79150 & 0.43750 \\ \mathrm{C} & -1.49650 & 1.43410 & -0.28150 \\ \mathrm{C} & 0.61000 & 2.47570 & -0.64550 \\ \mathrm{C} & -0.13020 & 2.23190 & -2.01140 \\ \mathrm{~N} & -1.45680 & 1.70770 & -1.64400 \\ \mathrm{H} & 0.69660 & 2.10750 & 2.24690 \\ \mathrm{H} & -1.12540 & 1.01390 & 3.55470 \\ \mathrm{H} & -3.14020 & 0.16260 & 2.39150 \\ \mathrm{H} & -3.39940 & 0.40910 & -0.05390 \\ \mathrm{H} & 0.40710 & 1.48810 & -2.61130 \\ \mathrm{C} & -2.12250 & 0.80880 & -2.57980 \\ \mathrm{H} & -2.03390 & 1.19720 & -3.59340 \\ \mathrm{H} & -1.68920 & -0.20240 & -2.55420 \\ \mathrm{H} & -3.18650 & 0.73990 & -2.34550 \\ \mathrm{C} & 2.01710 & 1.88080 & -0.55800 \\ \mathrm{H} & 2.66650 & 2.28380 & -1.33900 \\ \mathrm{H} & 2.46400 & 2.09280 & 0.41670 \\ \mathrm{H} & 1.96150 & 0.79590 & -0.67310 \\ \mathrm{C} & -0.15620 & 3.61040 & -2.75390 \\ \mathrm{~N} & 0.64420 & 3.95100 & -0.59590 \\ \mathrm{C} & 1.02460 & 3.69790 & -3.74550 \\ \mathrm{H} & 0.88590 & 2.96400 & -4.54480 \\ \mathrm{H} & 1.98080 & 3.49160 & -3.25760 \\ \mathrm{C} & 0.10630 & 4.62860 & -1.63550 \\ \mathrm{O} & -0.06260 & 5.84630 & -1.68600 \\ \mathrm{C} & -1.46800 & 3.93900 & -3.47180 \\ \mathrm{H} & -1.44070 & 4.97680 & -3.81250 \\ \mathrm{H} & -1.60230 & 3.29990 & -4.34870 \\ \mathrm{H} & -2.32520 & 3.81020 & -2.80890 \\ \mathrm{H} & 1.07770 & 4.69340 & -4.19360 \\ \mathrm{O} & 0.98120 & 4.58250 & 0.58950 \\ \mathrm{C} & 2.28370 & 5.21370 & 0.50530 \\ \mathrm{H} & 2.43890 & 5.67300 & 1.48300 \\ \mathrm{H} & 3.06080 & 4.47090 & 0.31180 \\ \mathrm{H} & 2.28380 & 5.97940 & -0.27290 \\ - & -----------------------\end{array}$

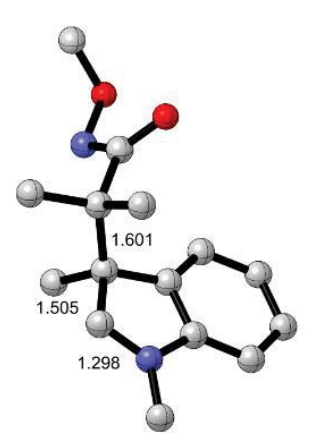

$\begin{array}{crrr}19 a & & & \\ \text { C } & -1.38840 & 2.09190 & 0.00020 \\ \text { C } & -2.59420 & 2.53220 & 0.53030 \\ \text { C } & -3.67740 & 1.64530 & 0.54340 \\ \text { C } & -3.55930 & 0.34150 & 0.04600 \\ \text { C } & -2.34740 & -0.12090 & -0.47710 \\ \text { C } & -1.29540 & 0.78400 & -0.47890 \\ \text { C } & -0.03540 & 2.76830 & -0.14800 \\ \text { C } & 0.75500 & 1.65400 & -0.77870 \\ \text { N } & 0.04850 & 0.57630 & -0.93490 \\ \text { H } & -2.69470 & 3.53640 & 0.92270\end{array}$

\begin{tabular}{rrrr} 
H & -4.62870 & 1.97640 & 0.94790 \\
$\mathrm{H}$ & -4.41770 & -0.32180 & 0.06930 \\
$\mathrm{H}$ & -2.24620 & -1.13220 & -0.85590 \\
$\mathrm{H}$ & 1.80640 & 1.67460 & -1.05080 \\
$\mathrm{C}$ & 0.52190 & -0.70660 & -1.47470 \\
$\mathrm{H}$ & 1.56190 & -0.60180 & -1.78040 \\
$\mathrm{H}$ & 0.42990 & -1.47040 & -0.69860 \\
$\mathrm{H}$ & -0.09910 & -0.97480 & -2.33220 \\
$\mathrm{C}$ & 0.60830 & 3.01420 & 1.24850 \\
$\mathrm{H}$ & 1.66690 & 3.26480 & 1.16000 \\
$\mathrm{H}$ & 0.08110 & 3.84460 & 1.71580 \\
$\mathrm{H}$ & 0.51480 & 2.11800 & 1.86720 \\
$\mathrm{C}$ & -0.07230 & 4.05200 & -1.10380 \\
$\mathrm{~N}$ & -0.93390 & 5.59190 & 0.55220 \\
$\mathrm{C}$ & 1.30330 & 4.74870 & -1.12410 \\
$\mathrm{H}$ & 2.09760 & 4.06860 & -1.45200 \\
$\mathrm{H}$ & 1.56290 & 5.15480 & -0.14830 \\
$\mathrm{C}$ & -1.18060 & 5.08920 & -0.64770 \\
$\mathrm{O}$ & -2.12960 & 5.35460 & -1.43770 \\
$\mathrm{C}$ & -0.40560 & 3.58290 & -2.53300 \\
$\mathrm{H}$ & -0.50790 & 4.44360 & -3.19380 \\
$\mathrm{H}$ & 0.39540 & 2.94930 & -2.92930 \\
$\mathrm{H}$ & -1.34510 & 3.02910 & -2.56760 \\
$\mathrm{H}$ & 1.27020 & 5.58220 & -1.83120 \\
$\mathrm{O}$ & -1.96420 & 6.55400 & 0.93710 \\
$\mathrm{C}$ & -1.55630 & 7.85800 & 0.53080 \\
$\mathrm{H}$ & -2.30170 & 8.56190 & 0.91170 \\
$\mathrm{H}$ & -0.57320 & 8.11250 & 0.95010 \\
$\mathrm{H}$ & -1.50750 & 7.93520 & -0.56180 \\
\hline
\end{tabular}

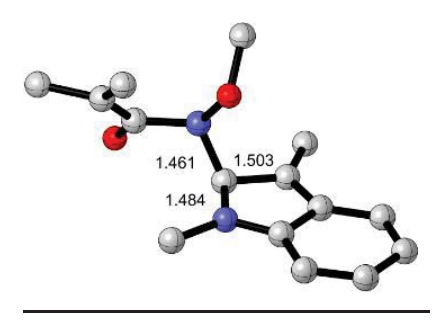

$\begin{array}{crrr}19 b & & & \\ \text { C } & -1.04910 & 1.55350 & 0.13530 \\ \text { C } & -2.31180 & 1.32590 & 0.77080 \\ \text { C } & -3.27120 & 0.63000 & 0.09190 \\ \text { C } & -3.01830 & 0.12830 & -1.22980 \\ \text { C } & -1.82470 & 0.31760 & -1.88480 \\ \text { C } & -0.81430 & 1.04930 & -1.20940 \\ \text { C } & 0.07940 & 2.21260 & 0.55070 \\ \text { C } & 1.09350 & 2.16970 & -0.55790 \\ \text { N } & 0.41260 & 1.37720 & -1.61220 \\ \text { H } & -2.49300 & 1.70630 & 1.77040 \\ \text { H } & -4.23910 & 0.44260 & 0.54380 \\ \text { H } & -3.80830 & -0.42540 & -1.72840 \\ \text { H } & -1.66970 & -0.07780 & -2.88150 \\ \text { H } & 2.01940 & 1.65710 & -0.25920 \\ \text { C } & 1.07330 & 0.98030 & -2.84750 \\ \text { H } & 1.80560 & 0.19010 & -2.65240 \\ \text { H } & 0.33880 & 0.62020 & -3.56990 \\ \text { H } & 1.59710 & 1.84090 & -3.26800 \\ \text { C } & 0.35350 & 2.90500 & 1.82850\end{array}$ 


$\begin{array}{rrrr}\text { H } & 0.88800 & 3.84030 & 1.63760 \\ \mathrm{H} & -0.55580 & 3.10650 & 2.39620 \\ \mathrm{H} & 1.01970 & 2.29300 & 2.45060 \\ \mathrm{C} & 2.77650 & 4.16690 & -3.00410 \\ \mathrm{~N} & 1.50400 & 3.51100 & -0.96610 \\ \mathrm{C} & 1.60770 & 4.52060 & -3.89910 \\ \mathrm{H} & 1.31750 & 5.58030 & -3.82220 \\ \mathrm{H} & 0.71190 & 3.93470 & -3.71180 \\ \mathrm{C} & 2.74590 & 3.60420 & -1.74720 \\ \mathrm{O} & 3.74410 & 3.15020 & -1.06370 \\ \mathrm{C} & 4.11730 & 4.50390 & -3.60510 \\ \mathrm{H} & 4.31070 & 3.91140 & -4.51230 \\ \mathrm{H} & 4.15280 & 5.55820 & -3.91830 \\ \mathrm{H} & 4.93590 & 4.32800 & -2.90620 \\ \mathrm{H} & 1.89480 & 4.36050 & -4.94580 \\ \mathrm{O} & 0.34330 & 4.17830 & -1.47180 \\ \mathrm{C} & 0.27400 & 5.51180 & -0.96130 \\ \mathrm{H} & -0.62020 & 5.94840 & -1.41200 \\ \mathrm{H} & 0.17710 & 5.52000 & 0.12690 \\ \mathrm{H} & 1.15280 & 6.08720 & -1.26110 \\ -----------------------------------\end{array}$

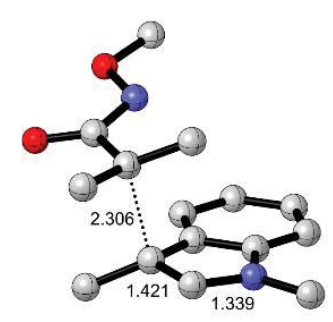

TS1‡

$\begin{array}{lrrr}\text { C } & -1.33760 & 2.15840 & 0.19360 \\ \text { C } & -2.40640 & 2.88020 & 0.72600 \\ \text { C } & -3.69380 & 2.35800 & 0.59390 \\ \text { C } & -3.92380 & 1.14000 & -0.06690 \\ \text { C } & -2.86980 & 0.40380 & -0.61140 \\ \text { C } & -1.59230 & 0.93450 & -0.45980 \\ \text { C } & 0.09960 & 2.41010 & 0.11670 \\ \text { C } & 0.62230 & 1.24920 & -0.51370 \\ \text { N } & -0.35550 & 0.41060 & -0.88040 \\ \text { H } & -2.24270 & 3.82600 & 1.23000 \\ \text { H } & -4.53660 & 2.90140 & 1.00870 \\ \text { H } & -4.93750 & 0.76200 & -0.15420 \\ \text { H } & -3.04560 & -0.53790 & -1.12060 \\ \text { H } & 1.65970 & 1.00140 & -0.70380 \\ \text { C } & -0.19620 & -0.85970 & -1.58790 \\ \text { H } & 0.86190 & -1.03040 & -1.78390 \\ \text { H } & -0.58460 & -1.67200 & -0.96820 \\ \text { H } & -0.74430 & -0.83480 & -2.53280 \\ \mathrm{C} & 0.88010 & 3.18150 & 1.15270 \\ \text { H } & 1.95230 & 3.12130 & 0.95370 \\ \text { H } & 0.58600 & 4.23050 & 1.18410 \\ \text { H } & 0.70060 & 2.74280 & 2.14020 \\ \mathrm{C} & 0.24580 & 3.81670 & -1.70500 \\ \text { N } & -1.80780 & 4.89160 & -1.55690 \\ \mathrm{C} & 1.72560 & 4.04220 & -1.76740 \\ \mathrm{H} & 2.28880 & 3.11700 & -1.89590 \\ \mathrm{H} & 2.08560 & 4.60790 & -0.91060 \\ \mathrm{C} & -0.54420 & 4.98140 & -1.16950\end{array}$

$\begin{array}{lrrr}\mathrm{O} & 0.01490 & 5.89790 & -0.47980 \\ \mathrm{C} & -0.32960 & 2.97690 & -2.81160 \\ \mathrm{H} & -0.43120 & 3.61620 & -3.69680 \\ \mathrm{H} & 0.33350 & 2.14920 & -3.07100 \\ \mathrm{H} & -1.32490 & 2.60360 & -2.57820 \\ \mathrm{H} & 1.90520 & 4.66650 & -2.65670 \\ \mathrm{O} & -2.55390 & 6.00320 & -1.11590 \\ \mathrm{C} & -3.92310 & 5.78510 & -1.46760 \\ \mathrm{H} & -4.46790 & 6.67470 & -1.14720 \\ \mathrm{H} & -4.03660 & 5.65390 & -2.54890 \\ \mathrm{H} & -4.31580 & 4.90020 & -0.95740\end{array}$

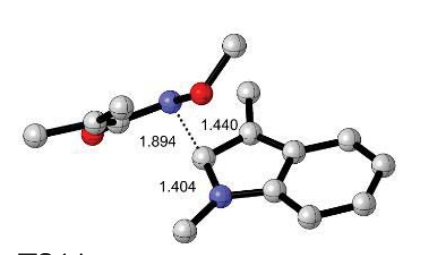

TS1A

\begin{tabular}{|c|c|c|c|}
\hline 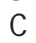 & -0.83040 & 1.90760 & 0.09670 \\
\hline & -2.14460 & 1.84060 & 0.62930 \\
\hline & -3.14420 & 1.28900 & -0.14240 \\
\hline & -2.87390 & 0.79710 & -1.45040 \\
\hline & -1.60600 & 0.84600 & -2.00730 \\
\hline & -0.57830 & 1.39940 & -1.22630 \\
\hline & 0.37900 & 2.41130 & 0.59100 \\
\hline & 1.35000 & 2.27690 & -0.46380 \\
\hline & 0.74880 & 1.54270 & -1.49920 \\
\hline & -2.35430 & 2.21870 & 1.62490 \\
\hline & -4.15650 & 1.22590 & 0.2432 \\
\hline & -3.68930 & 0.37250 & -2.0278 \\
\hline & -1.42160 & 0.47280 & $-3.0087 C$ \\
\hline & 2.41440 & 2.13040 & $-0.2967 C$ \\
\hline & 1.40610 & 1.19080 & -2.7494 \\
\hline & 2.47730 & 1.09310 & -2.5745 \\
\hline & 1.02290 & 0.23 & -3.11000 \\
\hline & 1.23920 & 1.95 & -3.5120 \\
\hline & 0.65700 & 3.09 & 1.8814 \\
\hline & 1.07790 & 4.08 & 1.6864 \\
\hline & -0.24430 & 3.20630 & 2.4867 \\
\hline & 1.40180 & 2.54690 & 2.4707 \\
\hline & 2.75860 & 4.45 & -3.2259 \\
\hline & 1.57430 & 4.05 & -1.0848 \\
\hline & 1.60670 & 4.97530 & -4.0479 \\
\hline & 1.059 & 5.77 & -3.5422 \\
\hline & 0.86210 & 4.20 & -4.2709 \\
\hline & 2.74540 & 3.997 & -1.8978 \\
\hline & 3.78240 & 3.58 & -1.2622 \\
\hline & 4.09420 & 4.56430 & -3.8998 \\
\hline & 4.041 & 4.11 & -4.9025 \\
\hline & 4.372 & 5.61 & \\
\hline & 4.88430 & 4.07750 & -3.3312 \\
\hline & 1.98040 & 5.36740 & $-4.997 \varepsilon$ \\
\hline & 0.41640 & 4.34740 & \\
\hline & -0.44370 & 5.20300 & -1.0050 \\
\hline & -1.33880 & 5.34060 & -1.6132 \\
\hline & -0.71270 & 4.73390 & -0.0574 \\
\hline & 0.04210 & 6.16550 & -0.8219 \\
\hline
\end{tabular}




\begin{tabular}{|c|c|c|c|}
\hline \multicolumn{4}{|c|}{$20 a$} \\
\hline C & -0.16960 & 1.50310 & 0.28670 \\
\hline C & -0.21570 & 1.27460 & 1.69920 \\
\hline C & -1.38950 & 0.84270 & 2.24940 \\
\hline C & -2.54890 & 0.62000 & 1.43080 \\
\hline C & -2.55650 & 0.82790 & 0.07230 \\
\hline C & -1.35170 & 1.28840 & -0.51940 \\
\hline C & 0.85040 & 1.88890 & -0.55460 \\
\hline C & 0.30110 & 2.00870 & -1.94460 \\
\hline $\mathrm{N}$ & -1.09070 & 1.54830 & -1.80230 \\
\hline $\mathrm{H}$ & 0.66300 & 1.44480 & 2.31250 \\
\hline $\mathrm{H}$ & -1.46320 & 0.66010 & 3.31600 \\
\hline $\mathrm{H}$ & -3.45850 & 0.27470 & 1.91430 \\
\hline $\mathrm{H}$ & -3.45170 & 0.66150 & -0.51600 \\
\hline $\mathrm{H}$ & 0.83980 & 1.34450 & -2.63380 \\
\hline C & -1.96710 & 1.13920 & -2.90410 \\
\hline $\mathrm{H}$ & -1.38210 & 1.04680 & -3.81720 \\
\hline $\mathrm{H}$ & -2.40190 & 0.16300 & -2.67470 \\
\hline $\mathrm{H}$ & -2.77150 & 1.86300 & -3.06060 \\
\hline C & 2.25490 & 2. 18470 & -0.19390 \\
\hline $\mathrm{H}$ & 2.47390 & 3.24600 & -0.36540 \\
\hline $\mathrm{H}$ & 2.46330 & 1.94800 & 0.84970 \\
\hline $\mathrm{H}$ & 2.93730 & 1. 62320 & -0.84050 \\
\hline C & 0.44750 & 3.48550 & -2.52620 \\
\hline $\mathrm{N}$ & 2.44040 & 4.83530 & -2.55570 \\
\hline C & -0.34840 & 3.64880 & -3.83800 \\
\hline $\mathrm{H}$ & -1.42530 & 3.63150 & -3.66280 \\
\hline $\mathrm{H}$ & -0.08730 & 2.88230 & -4.56860 \\
\hline C & 1.95120 & 3.66280 & -2.93040 \\
\hline 0 & 2.49930 & 2.73640 & -3.59430 \\
\hline C & -0.08130 & 4.50330 & -1.50320 \\
\hline $\mathrm{H}$ & -0.08940 & 5.50300 & -1.93800 \\
\hline $\mathrm{H}$ & -1.10820 & 4. 24840 & -1.22060 \\
\hline $\mathrm{H}$ & 0.52670 & 4.54430 & -0.59910 \\
\hline $\mathrm{H}$ & -0.10170 & 4.62340 & -4.26750 \\
\hline 0 & 3.78960 & 5.03050 & -3.08110 \\
\hline C & 4.74740 & 4.50960 & -2.16140 \\
\hline $\mathrm{H}$ & 5.73540 & 4.76210 & -2.55580 \\
\hline $\mathrm{H}$ & 4. 62990 & 4.96350 & -1.16910 \\
\hline $\mathrm{H}$ & 4.65720 & 3.42200 & -2.07270 \\
\hline
\end{tabular}

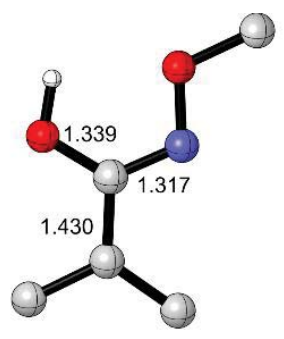

$\begin{array}{rrrr}22 & & & \\ \mathrm{C} & -0.38120 & 4.89870 & -1.12400 \\ \mathrm{~N} & 1.22530 & 3.82480 & -2.42850 \\ \mathrm{C} & -1.46720 & 4.08270 & -1.69150 \\ \mathrm{H} & -1.98990 & 3.58310 & -0.85950 \\ \mathrm{H} & -1.14740 & 3.35520 & -2.43170 \\ \mathrm{C} & 0.98520 & 4.74580 & -1.51880 \\ \mathrm{O} & 1.91190 & 5.52860 & -0.95300 \\ \mathrm{C} & -0.70830 & 5.91310 & -0.11020 \\ \mathrm{H} & -0.36050 & 6.89990 & -0.45370 \\ \mathrm{H} & -1.77380 & 5.94330 & 0.11580 \\ \mathrm{H} & -0.12820 & 5.71590 & 0.80470 \\ \mathrm{H} & -2.21820 & 4.76650 & -2.11940 \\ \mathrm{O} & 2.51610 & 3.76020 & -2.72070 \\ \mathrm{C} & 2.82070 & 2.74530 & -3.72710 \\ \mathrm{H} & 3.90090 & 2.80730 & -3.85070 \\ \mathrm{H} & 2.52000 & 1.76560 & -3.35080 \\ \mathrm{H} & 2.30000 & 2.99290 & -4.65410 \\ \mathrm{H} & 2.80700 & 5.34080 & -1.29690\end{array}$

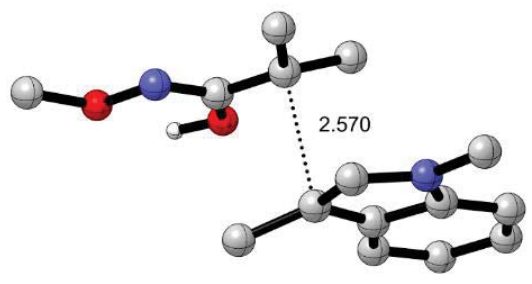

TS1‡(H+)

$\begin{array}{rrrr}\mathrm{C} & -1.68950 & 2.14650 & 0.03940 \\ \mathrm{C} & -3.06140 & 2.23390 & 0.30090 \\ \mathrm{C} & -3.89480 & 1.22310 & -0.17740 \\ \mathrm{C} & -3.37920 & 0.13470 & -0.90340 \\ \mathrm{C} & -2.01400 & 0.02430 & -1.17250 \\ \mathrm{C} & -1.19500 & 1.04050 & -0.69000 \\ \mathrm{C} & -0.55540 & 2.99410 & 0.35870 \\ \mathrm{C} & 0.56880 & 2.31510 & -0.16400 \\ \mathrm{~N} & 0.19850 & 1.18930 & -0.79930 \\ \mathrm{H} & -3.47060 & 3.06950 & 0.85900 \\ \mathrm{H} & -4.96190 & 1.27270 & 0.01450 \\ \mathrm{H} & -4.05460 & -0.63710 & -1.25850 \\ \mathrm{H} & -1.61770 & -0.81860 & -1.72880 \\ \mathrm{H} & 1.61380 & 2.58490 & -0.07620 \\ \mathrm{C} & 1.09100 & 0.21700 & -1.43170 \\ \mathrm{H} & 2.10970 & 0.60290 & -1.41200 \\ \mathrm{H} & 1.05030 & -0.72770 & -0.88330 \\ \mathrm{H} & 0.78780 & 0.04640 & -2.46700 \\ \mathrm{C} & -0.49270 & 3.99680 & 1.46870 \\ \mathrm{H} & 0.37230 & 4.65570 & 1.36780 \\ \mathrm{H} & -1.39970 & 4.60370 & 1.51090\end{array}$




\begin{tabular}{lrrr}
$\mathrm{H}$ & -0.40550 & 3.47610 & 2.42970 \\
$\mathrm{C}$ & -0.52080 & 4.53070 & -1.70050 \\
$\mathrm{~N}$ & -0.22650 & 6.54630 & -0.52380 \\
$\mathrm{C}$ & 0.91630 & 4.59820 & -2.12270 \\
$\mathrm{H}$ & 1.24770 & 3.64280 & -2.53060 \\
$\mathrm{H}$ & 1.58210 & 4.92280 & -1.32520 \\
$\mathrm{C}$ & -1.06520 & 5.64520 & -0.94520 \\
$\mathrm{O}$ & -2.39600 & 5.68390 & -0.74850 \\
$\mathrm{C}$ & -1.43920 & 3.74580 & -2.56550 \\
$\mathrm{H}$ & -1.50600 & 4.28570 & -3.52510 \\
$\mathrm{H}$ & -1.02260 & 2.76250 & -2.79640 \\
$\mathrm{H}$ & -2.44430 & 3.65010 & -2.16090 \\
$\mathrm{H}$ & 0.98360 & 5.34220 & -2.92990 \\
$\mathrm{O}$ & -0.88110 & 7.56170 & 0.12750 \\
$\mathrm{C}$ & 0.04680 & 8.58760 & 0.54700 \\
$\mathrm{H}$ & -0.56370 & 9.33030 & 1.05910 \\
$\mathrm{H}$ & 0.79050 & 8.16750 & 1.22830 \\
$\mathrm{H}$ & 0.53540 & 9.03170 & -0.32460 \\
$\mathrm{H}$ & -2.64540 & 6.49550 & -0.26740 \\
\hline------------------------------------
\end{tabular}

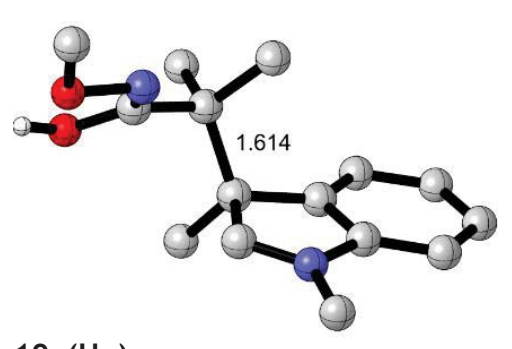

\begin{tabular}{rrrr}
\multicolumn{1}{c}{ 19a(H+) } & & \\
C & -1.48710 & 2.05440 & -0.03630 \\
C & -2.75080 & 2.41130 & 0.41720 \\
C & -3.79820 & 1.49310 & 0.26340 \\
C & -3.58910 & 0.23800 & -0.31950 \\
C & -2.31670 & -0.14750 & -0.75260 \\
C & -1.30340 & 0.78500 & -0.58780
\end{tabular}

\begin{tabular}{|c|c|c|c|}
\hline C & -0.15270 & 2.79050 & -0.05250 \\
\hline C & 0.74000 & 1.72180 & -0.62820 \\
\hline $\mathrm{N}$ & 0.08690 & 0.64170 & -0.91650 \\
\hline $\mathrm{H}$ & -2.94110 & 3.37280 & 0.88040 \\
\hline $\mathrm{H}$ & -4.79350 & 1. 76120 & 0.60360 \\
\hline $\mathrm{H}$ & -4.42110 & -0.44990 & -0.42890 \\
\hline $\mathrm{H}$ & -2.14300 & -1.12500 & -1.18920 \\
\hline $\mathrm{H}$ & 1.81480 & 1.78320 & -0.76810 \\
\hline C & 0.65430 & -0.59610 & -1.47350 \\
\hline $\mathrm{H}$ & 1.72410 & -0.46080 & -1.62950 \\
\hline $\mathrm{H}$ & 0.47460 & -1.41060 & -0.76820 \\
\hline $\mathrm{H}$ & 0.15600 & -0.80760 & -2.42220 \\
\hline C & 0.32720 & 3.09690 & 1. 39210 \\
\hline $\mathrm{H}$ & 1.29620 & 3. 59570 & 1. 38770 \\
\hline $\mathrm{H}$ & -0.40220 & 3.73870 & 1.88920 \\
\hline $\mathrm{H}$ & 0.40140 & 2. 16970 & 1.96660 \\
\hline C & -0.16070 & 4.07710 & -1.02620 \\
\hline $\mathrm{N}$ & 1.67410 & 4.11330 & -2.61990 \\
\hline C & -0.99710 & 3.76620 & -2.28720 \\
\hline $\mathrm{H}$ & -2.04930 & 3.66390 & -2.01980 \\
\hline $\mathrm{H}$ & -0.67340 & 2.85130 & -2.78610 \\
\hline C & 1.27450 & 4. 41490 & -1.43840 \\
\hline 0 & 2.03700 & 5.01680 & -0.50440 \\
\hline C & -0.77610 & 5.30220 & -0.31950 \\
\hline $\mathrm{H}$ & -0.79690 & 6.14110 & -1.02100 \\
\hline $\mathrm{H}$ & -1.80500 & 5.09720 & -0.01950 \\
\hline $\mathrm{H}$ & -0.20750 & 5.61330 & 0.55500 \\
\hline $\mathrm{H}$ & -0.90500 & 4. 58350 & -3.00370 \\
\hline 0 & 3.02640 & 4. 49050 & -2.80160 \\
\hline C & 3.31420 & 4. 56120 & -4.20640 \\
\hline $\mathrm{H}$ & 4.37810 & 4. 79040 & -4.27870 \\
\hline $\mathrm{H}$ & 3.10310 & 3.60330 & -4.68950 \\
\hline $\mathrm{H}$ & 2.72980 & 5. 35420 & -4.68300 \\
\hline $\mathrm{H}$ & 2.91230 & 5.22100 & -0.88670 \\
\hline
\end{tabular}




\section{References.}

(1) (a) Becke, A. D. J. Chem. Phys. 1993, 98, 5648-5652; (b) Becke, A. D. J. Chem. Phys. 1993, 98, 1372-1377; (c) Lee, C.; Yang, W.; Parr, R. G. Phys. Rev. B 1988, 37, 785-789; (d) Stephens, P. J.; Devlin, F. J.; Chabalowski, C. F.; Frisch, M. J. J. Phys. Chem. 1994, 98, 11623-11627.

(2) (a) Grimme, S.; Antony, J.; Ehrlich, S.; Krieg, H. J. Chem. Phys. 2010, 132, 154104; (b) Goerigk, L.; Grimme, S. Phys. Chem. Chem. Phys. 2011, 13, 66706688.

(3) (a) Frisch, M. J.; Pople, J. A.; Binkley, J. S. J. Chem. Phys. 1984, 80, 32653269; (b) Clark, T.; Chandrasekhar, J.; Spitznagel, G. W.; Schleyer, P. v. R. J. Comput. Chem. 1983, 4, 294-301; (c) McLean, A. D.; Chandler, G. S. J, Chem. Phys. 1980, 72, 5639-5648; (d) Krishnan, R.; Binkley, J. S.; Seeger, R.; Pople, J. A. J. Chem. Phys. 1980, 72, 650-654.

(4) Jaguar, versions 7.0-8.3, Schrödinger, LLC, New York, NY: 2007-2014.

(5) (a) Marten, B.; Kim, K.; Cortis, C.; Friesner, R. A.; Murphy, R. B.; Ringnalda, M. N.; Sitkoff, D.; Honig, B. J. Phys. Chem. 1996, 100, 11775-11788; (b) Tannor, D. J.; Marten, B.; Murphy, R.; Friesner, R. A.; Sitkoff, D.; Nicholls, A.; Honig, B.; Ringnalda, M.; Goddard, W. A. J. Am. Chem. Soc. 1994, 116, 11875-11882.

(6) Legault, C. Y. CYLview, 1.0b, Université de Sherbrooke: Université de Sherbrooke, 2009. 


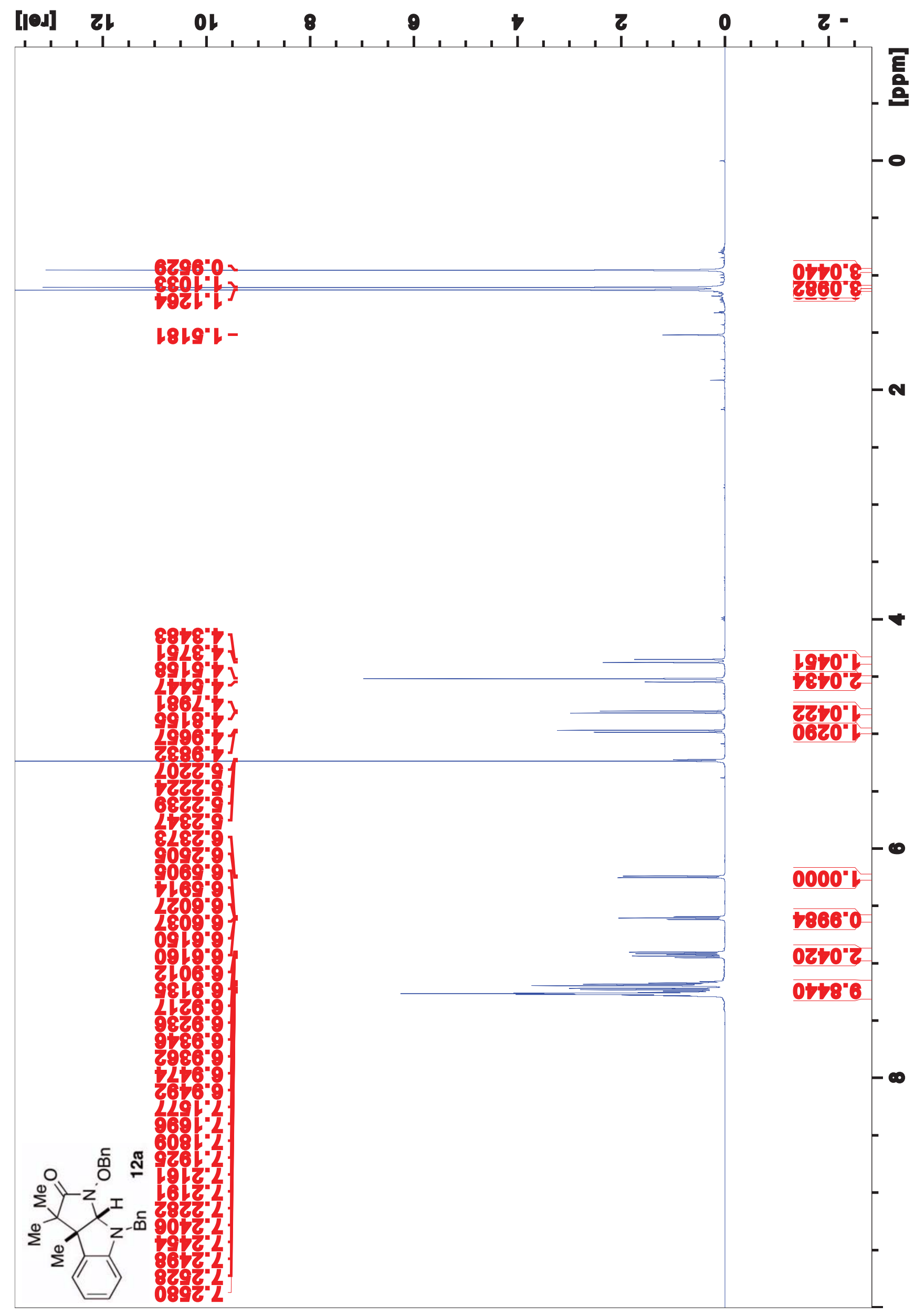




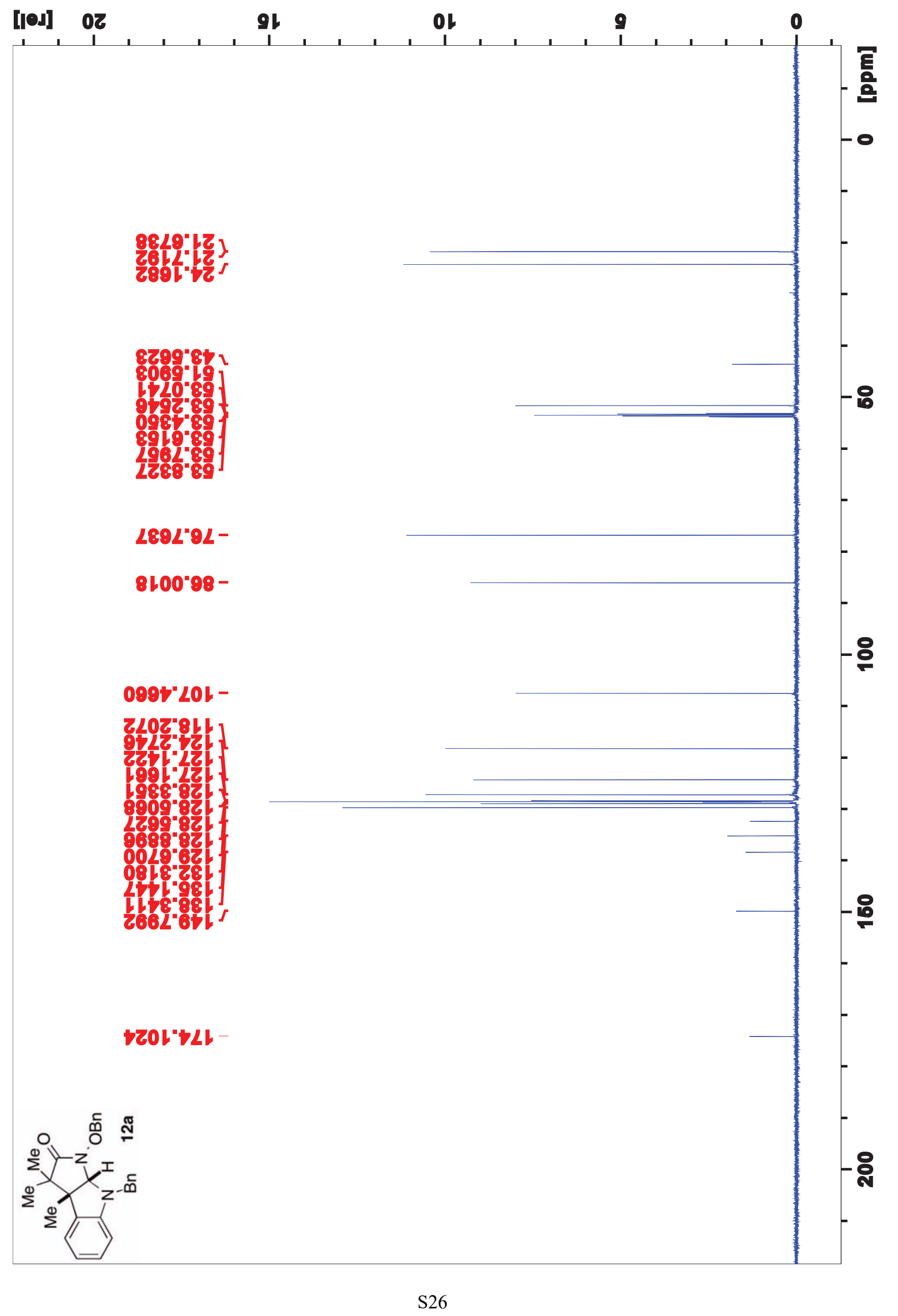




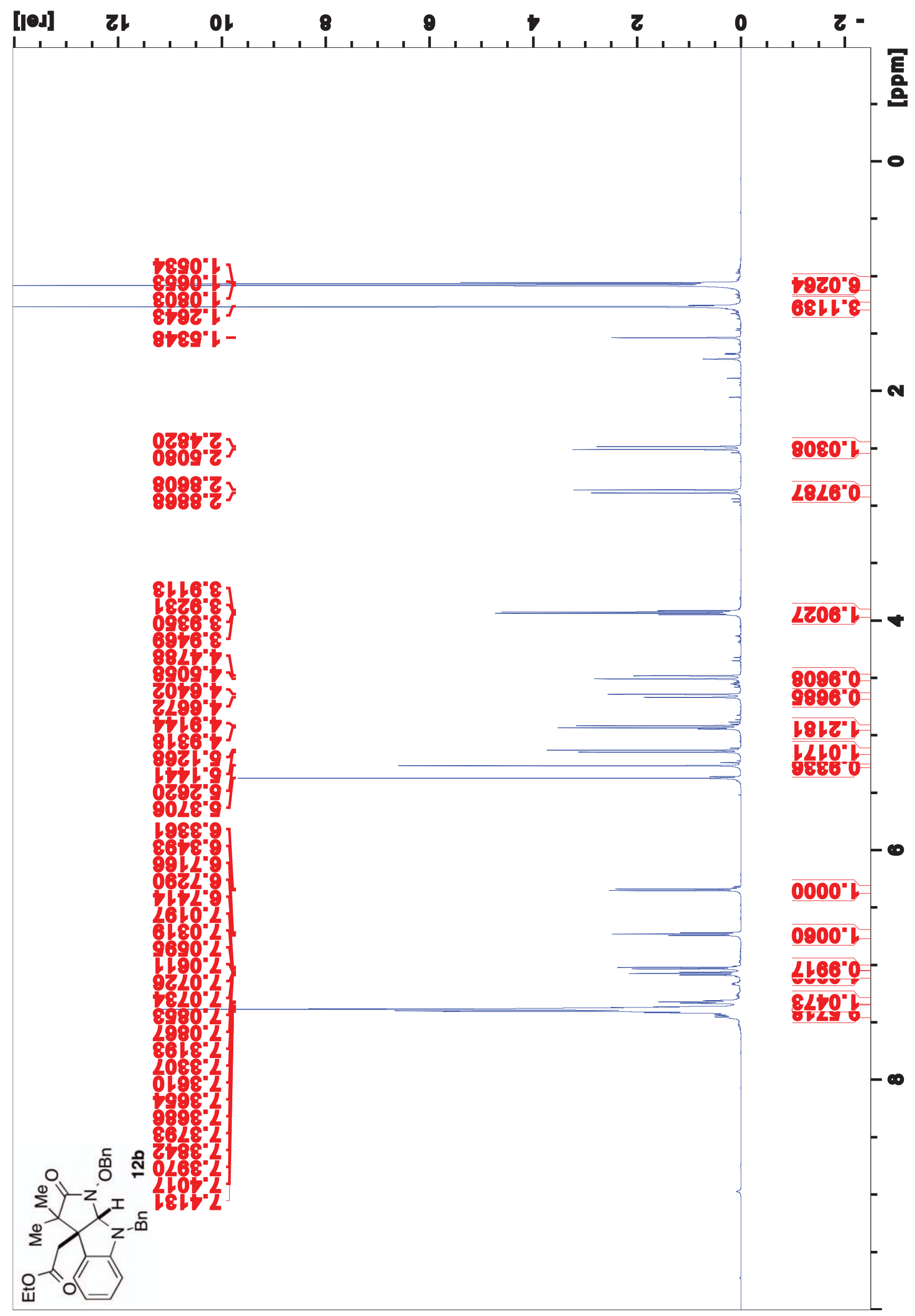




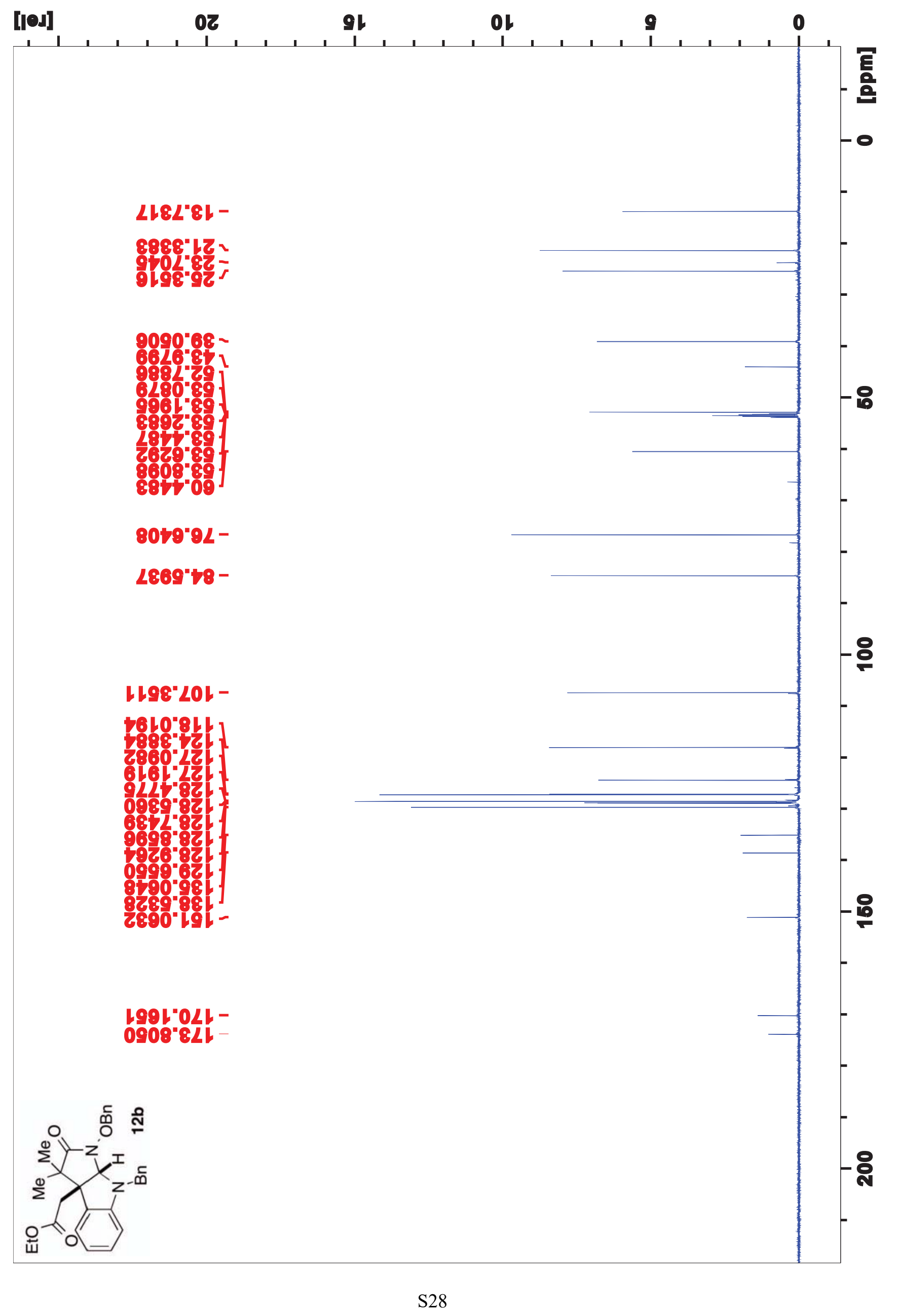




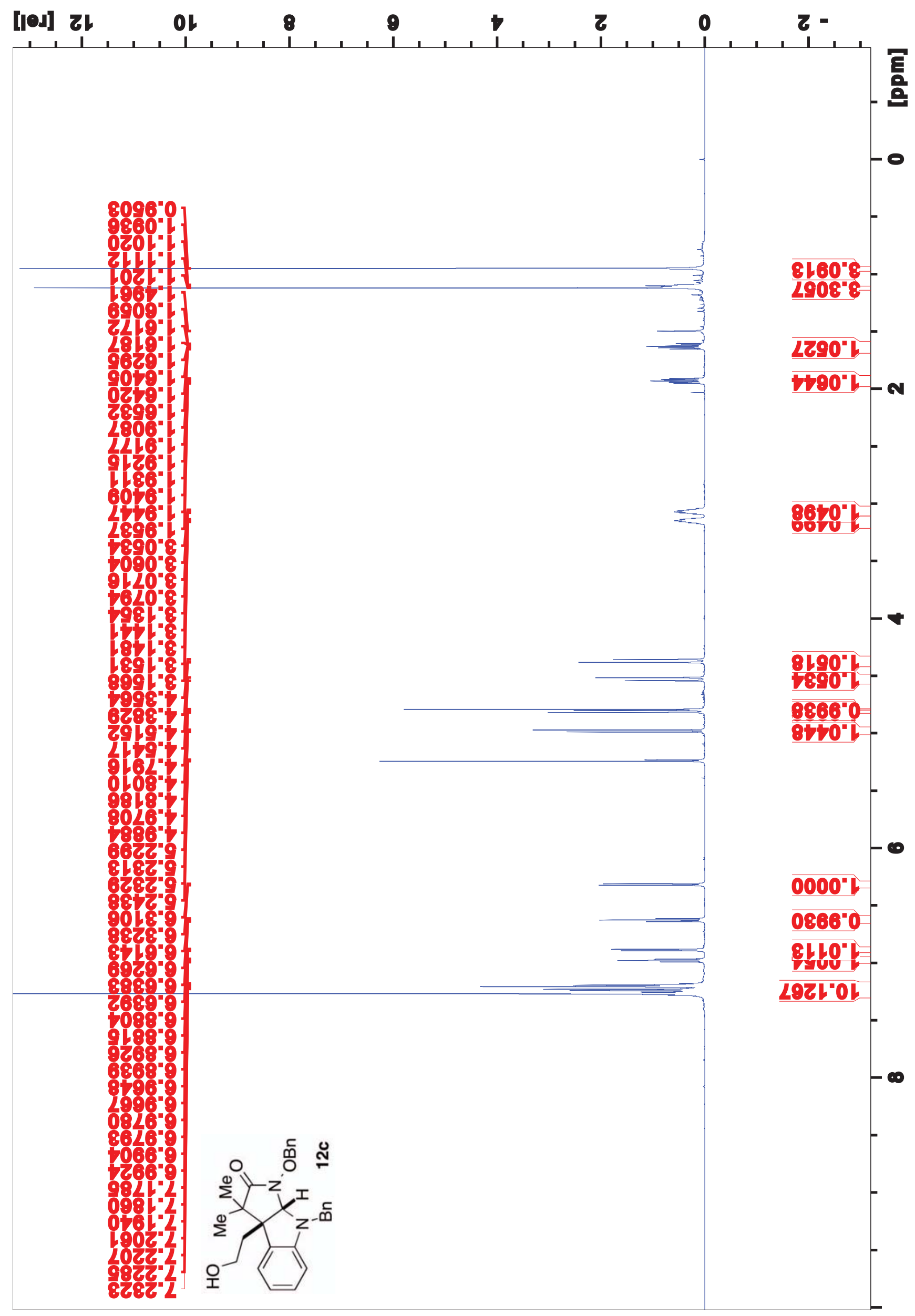




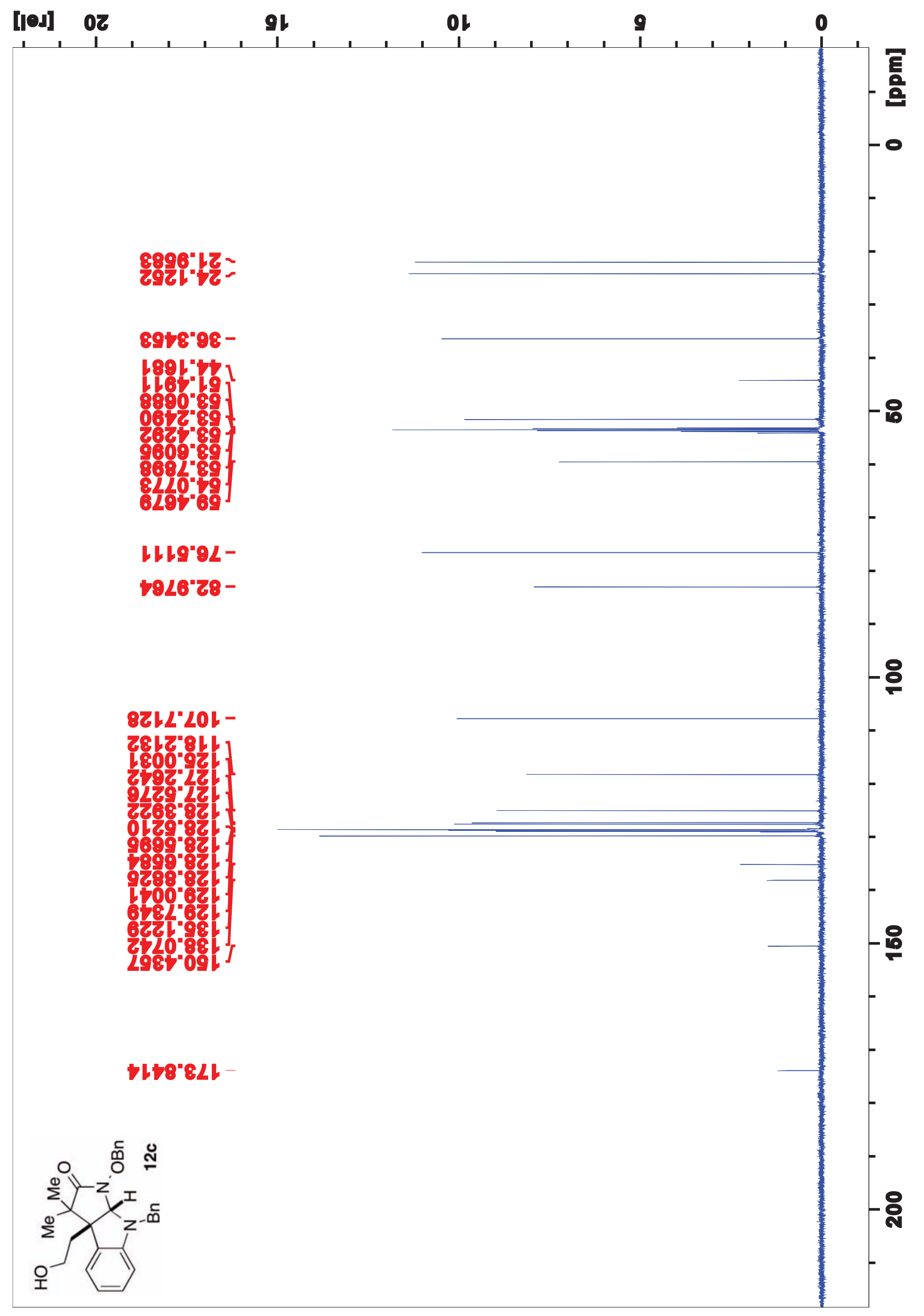




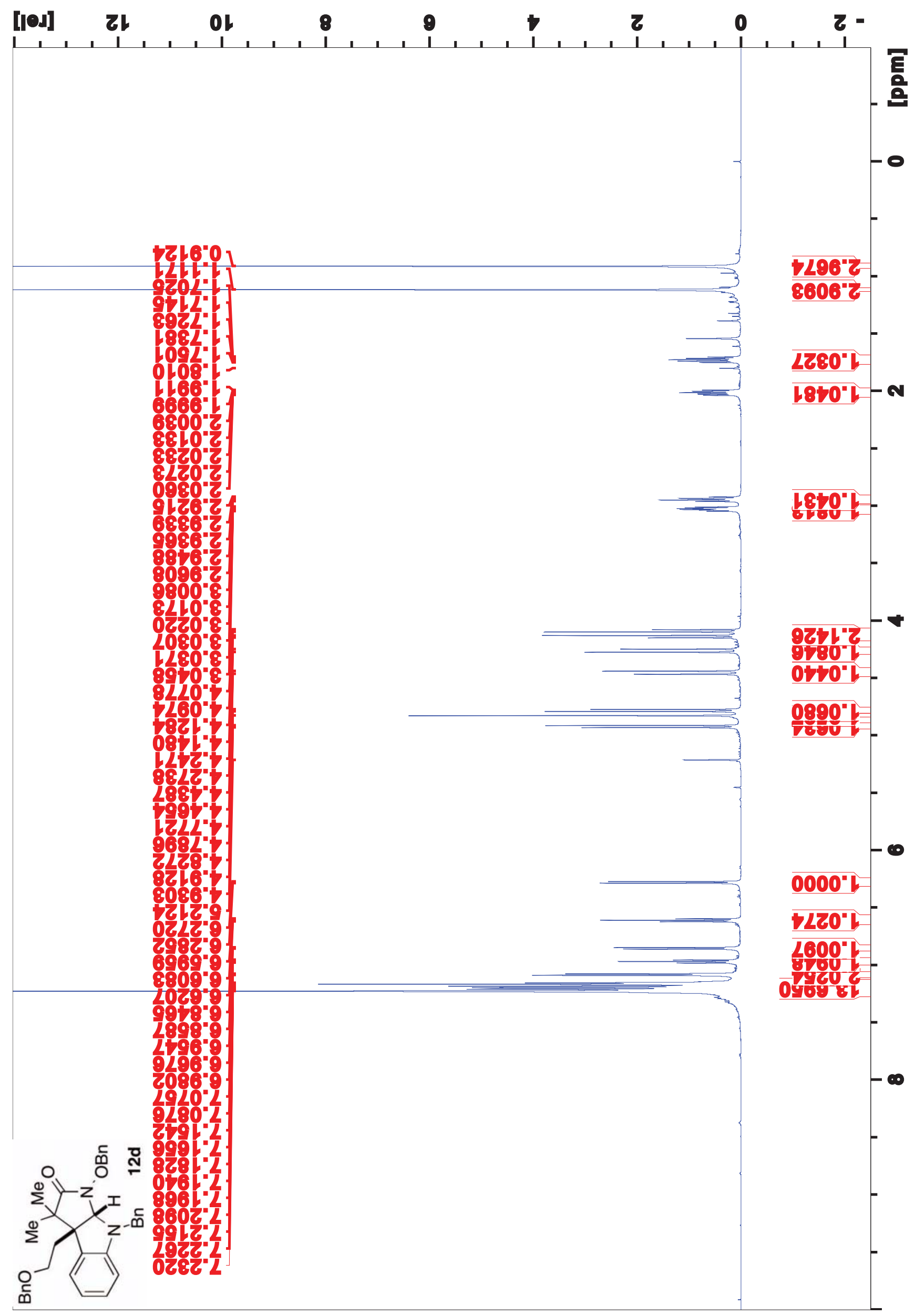




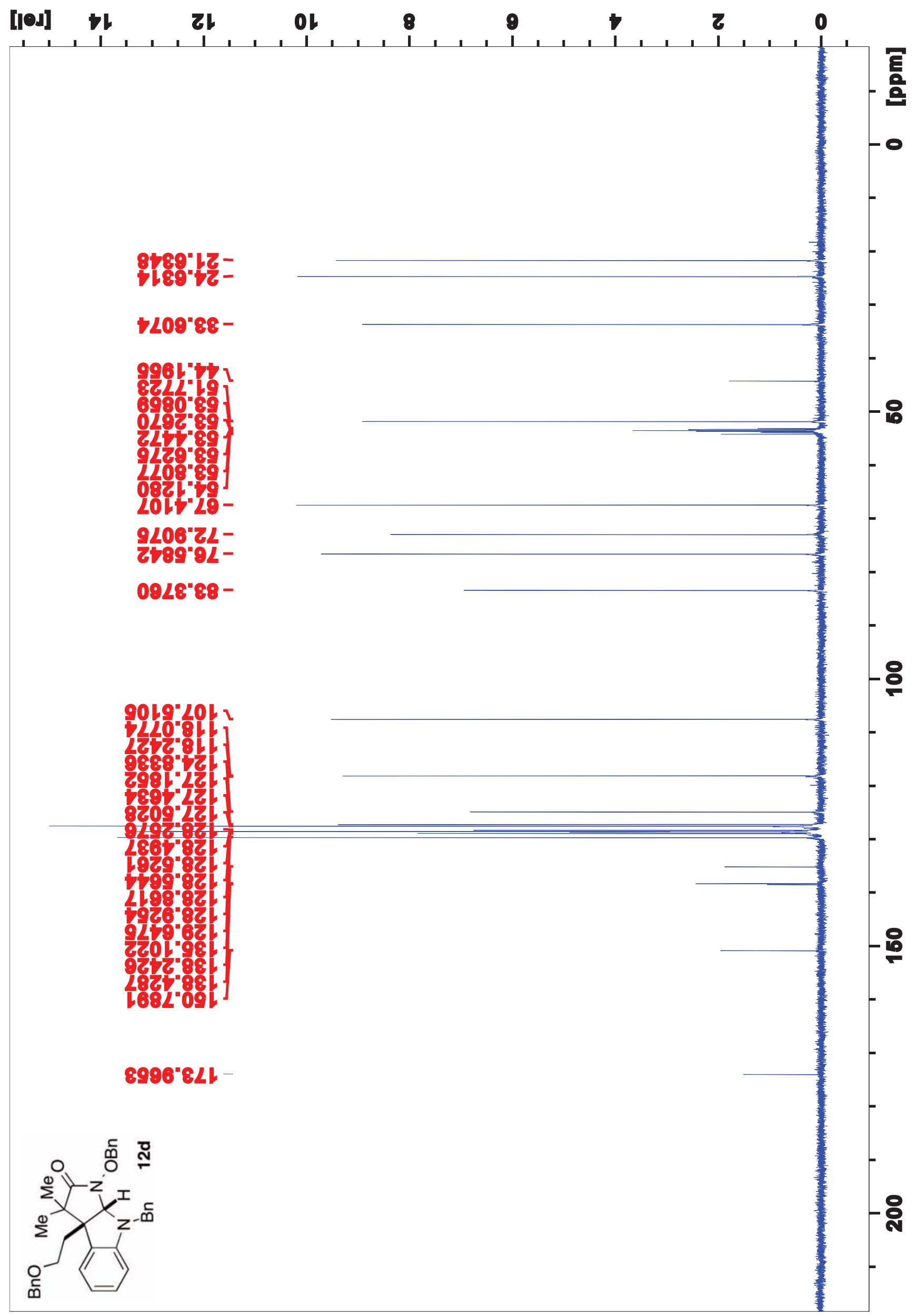




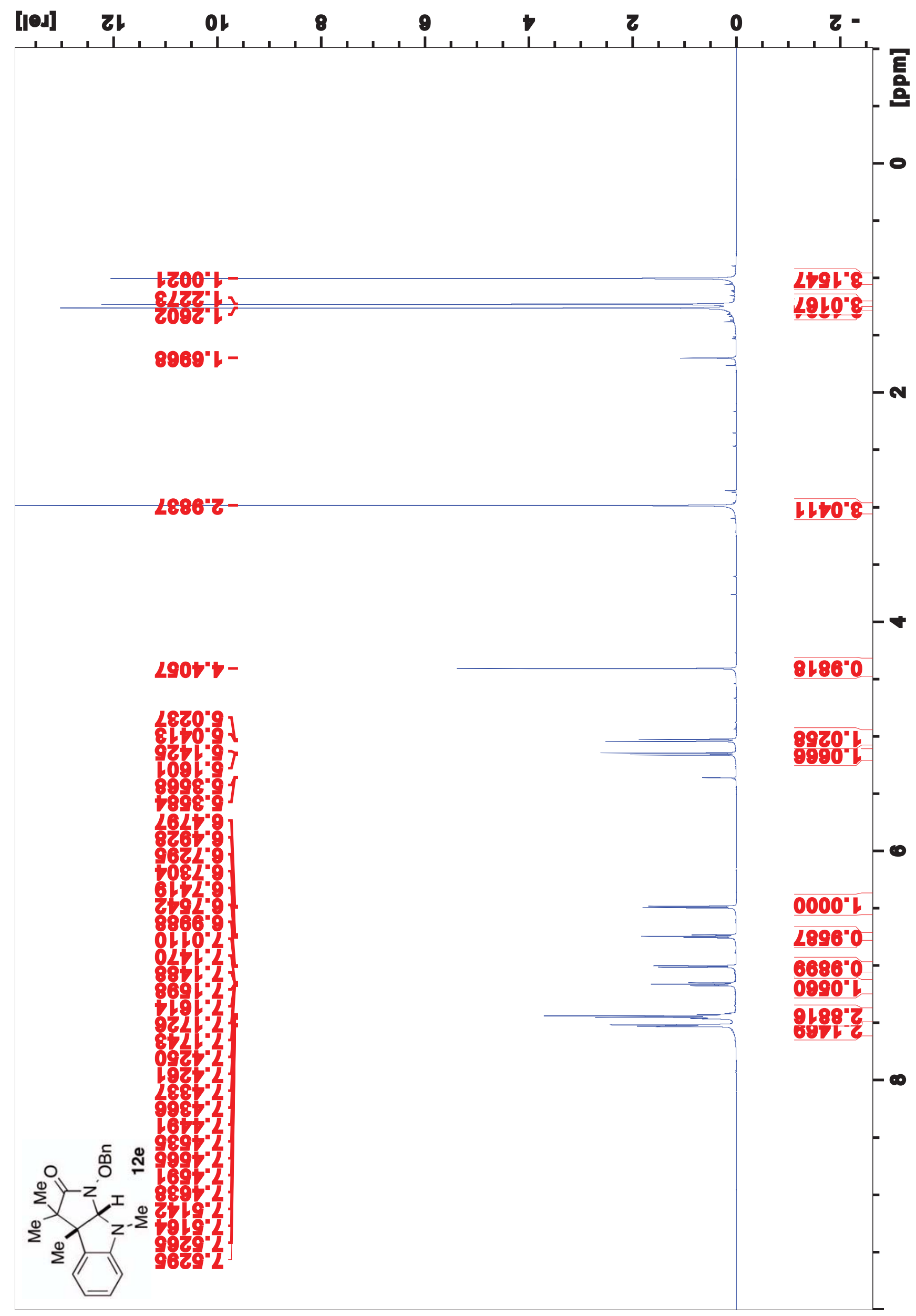




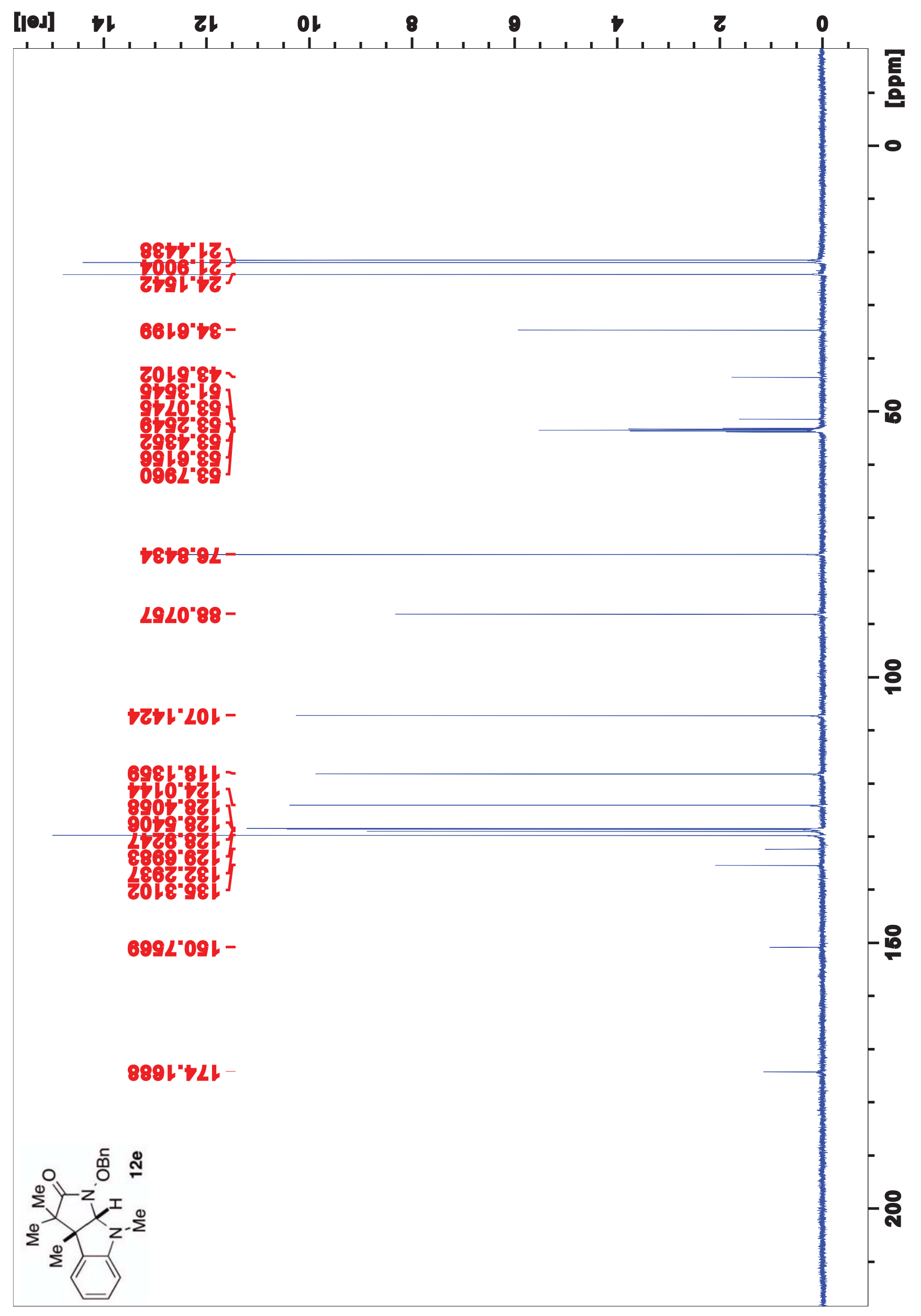




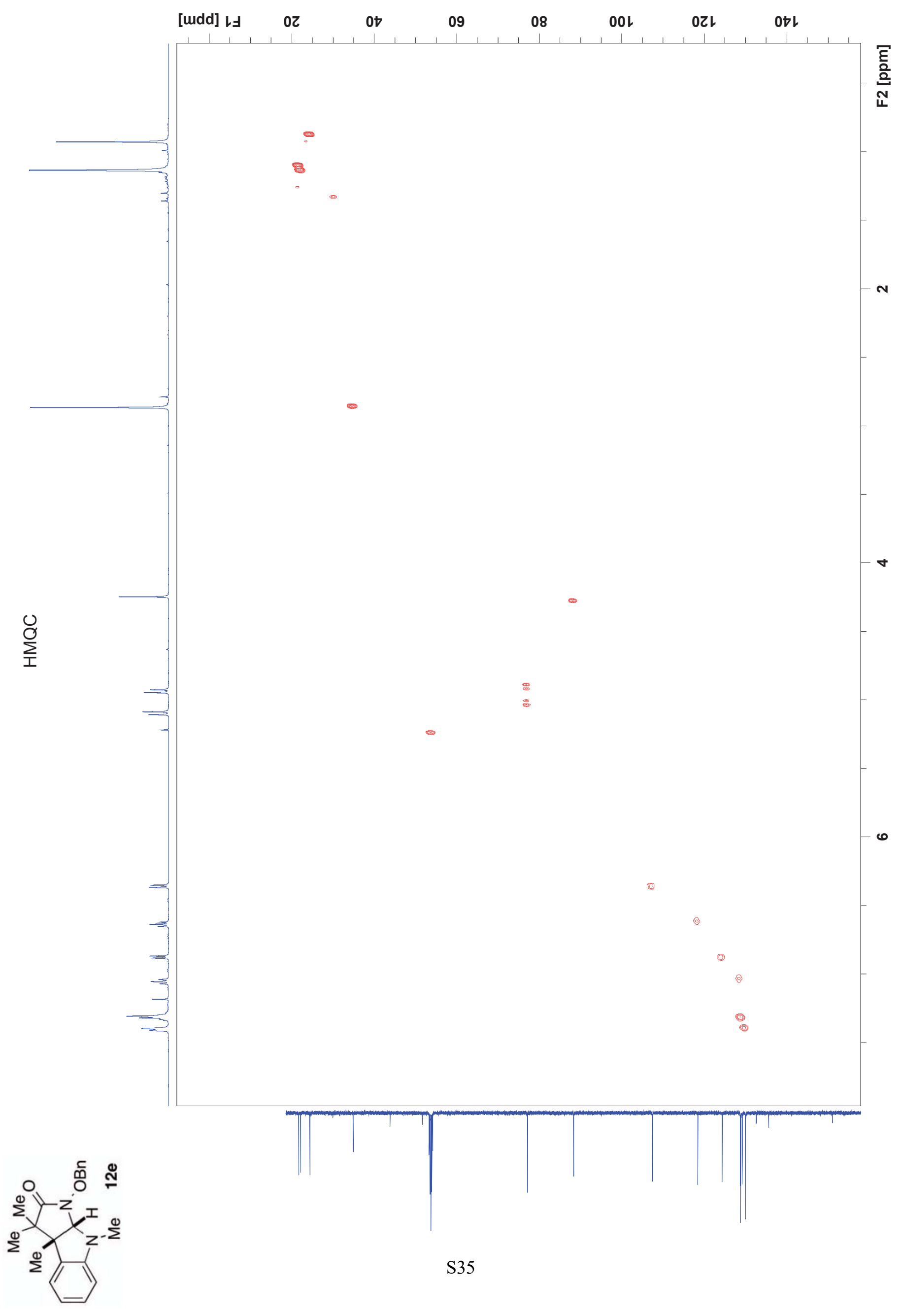




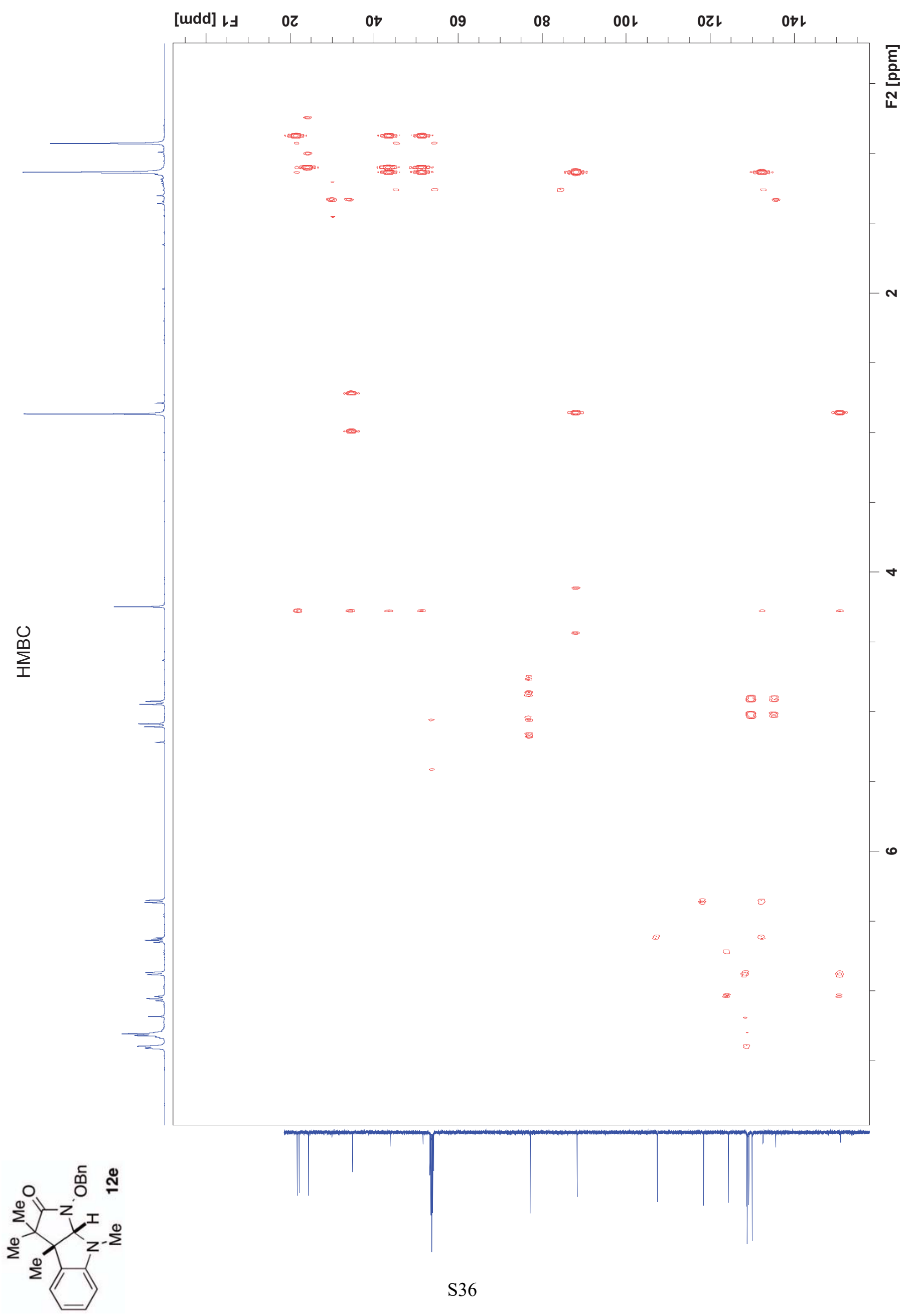




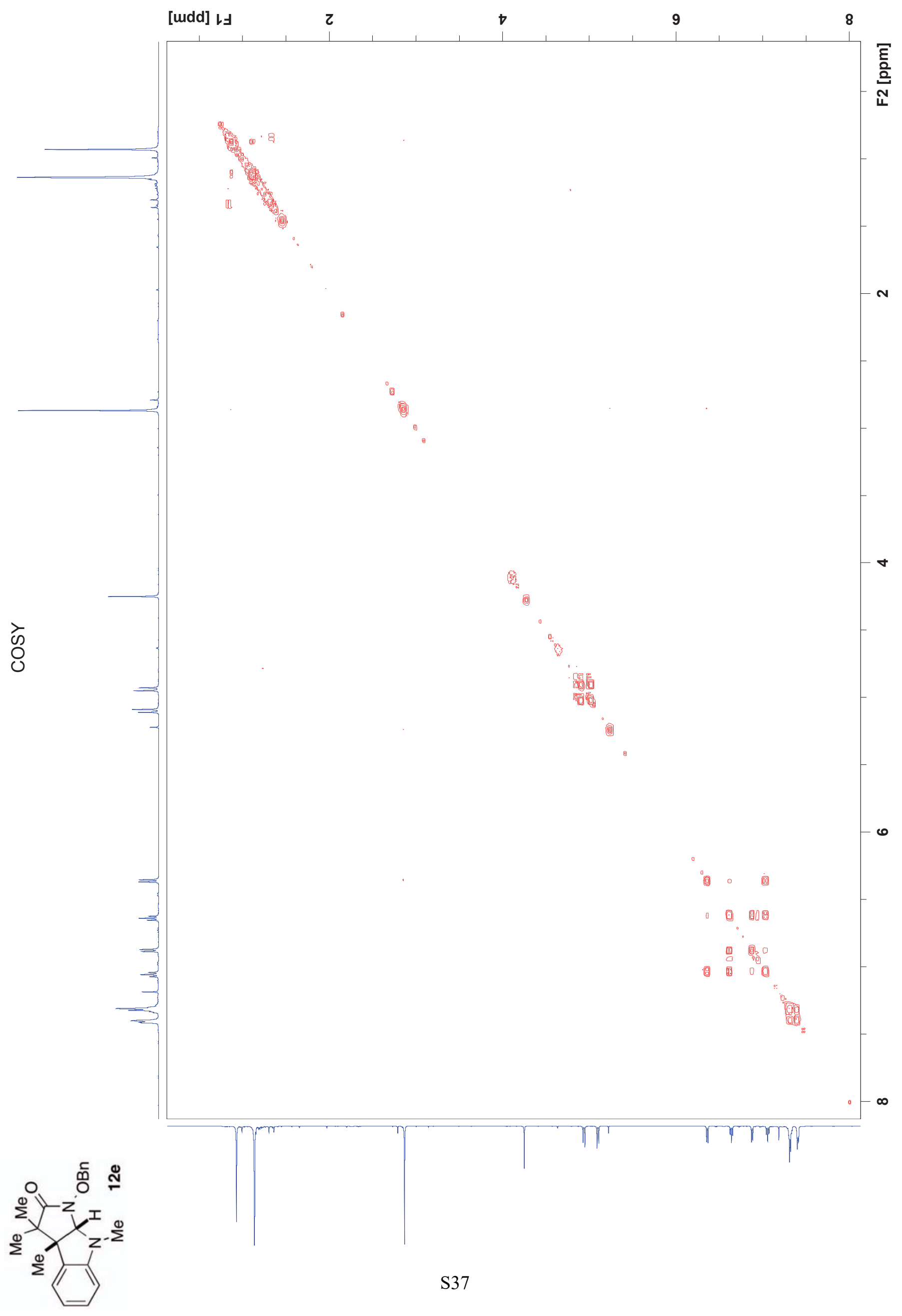




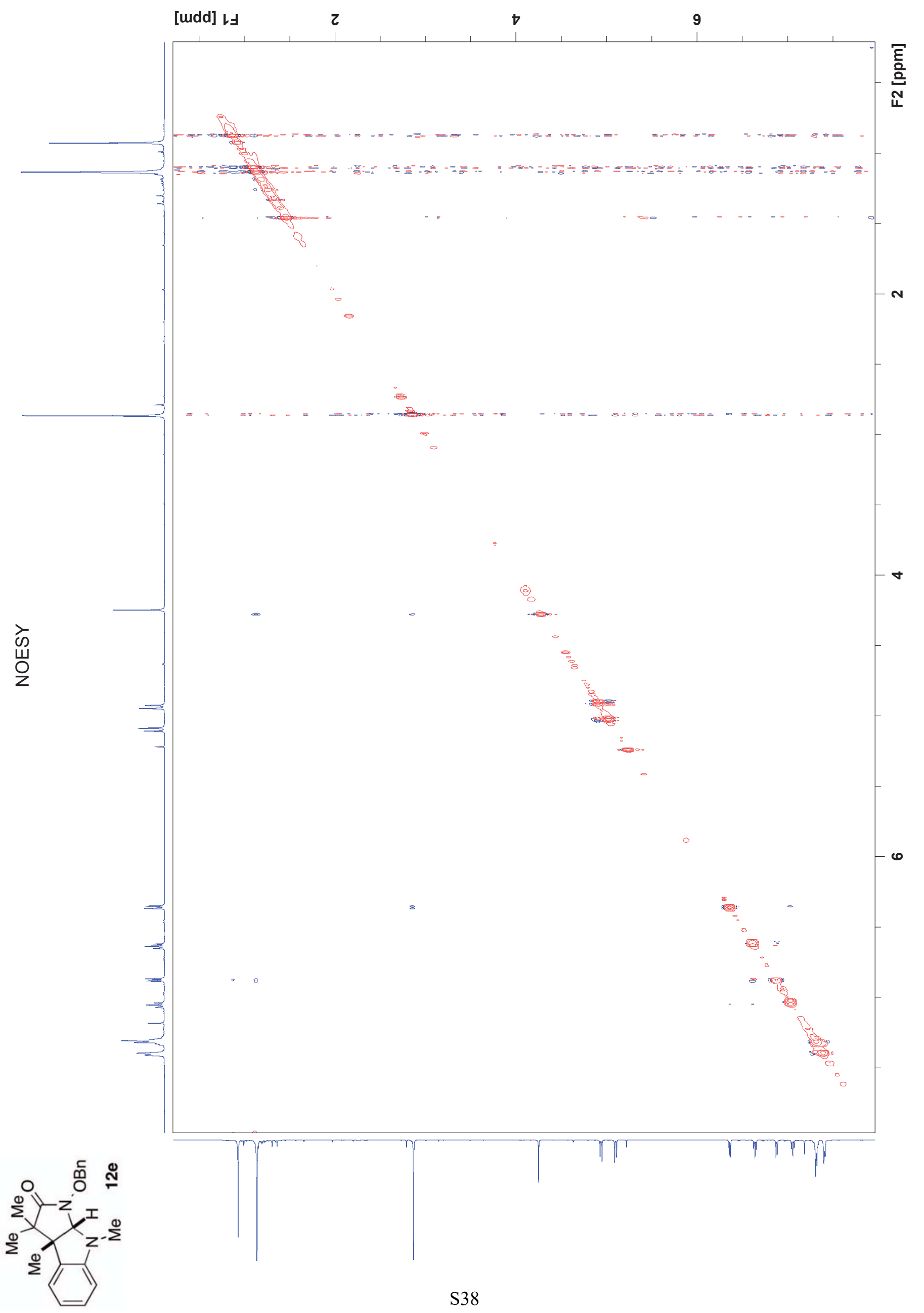




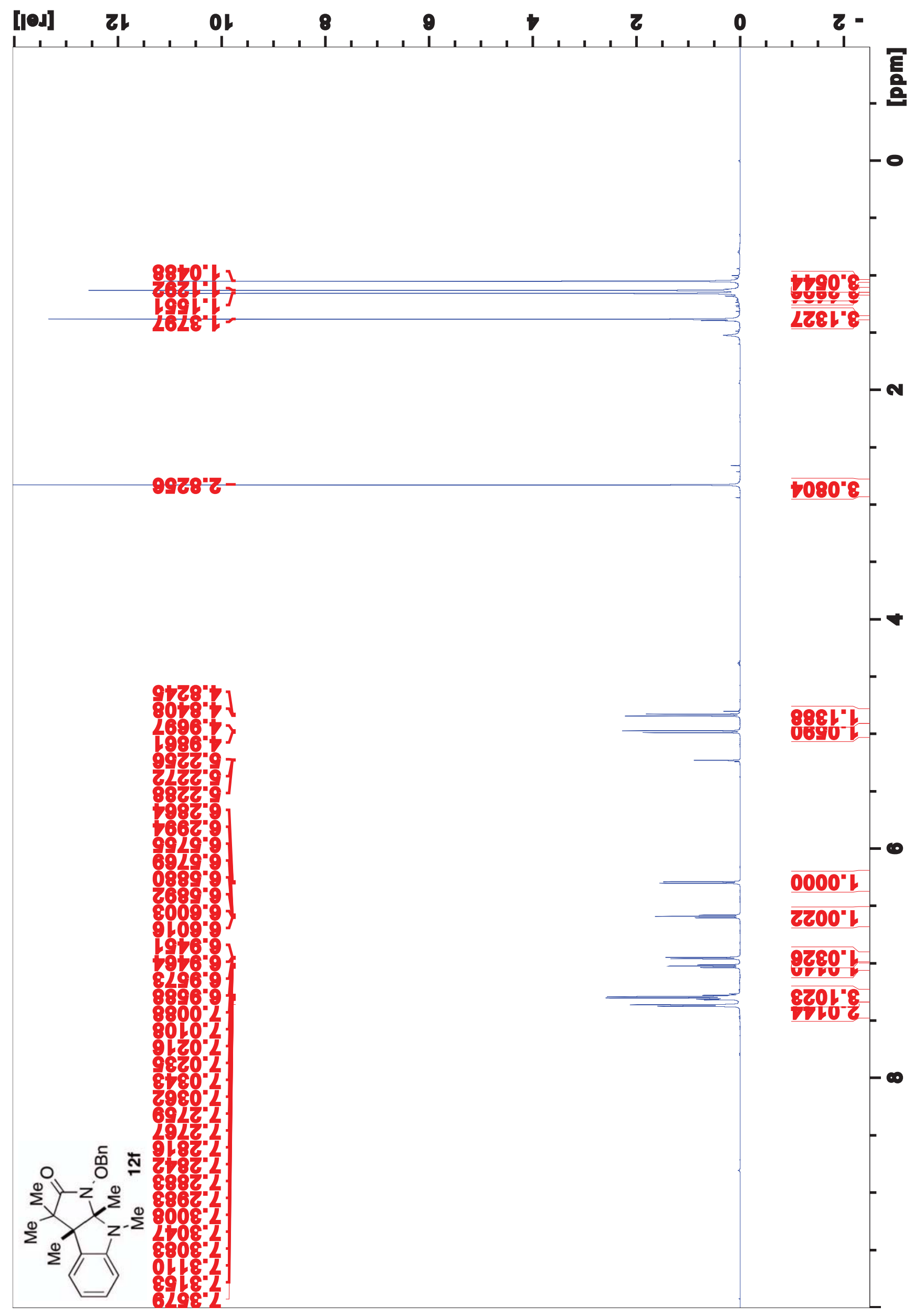




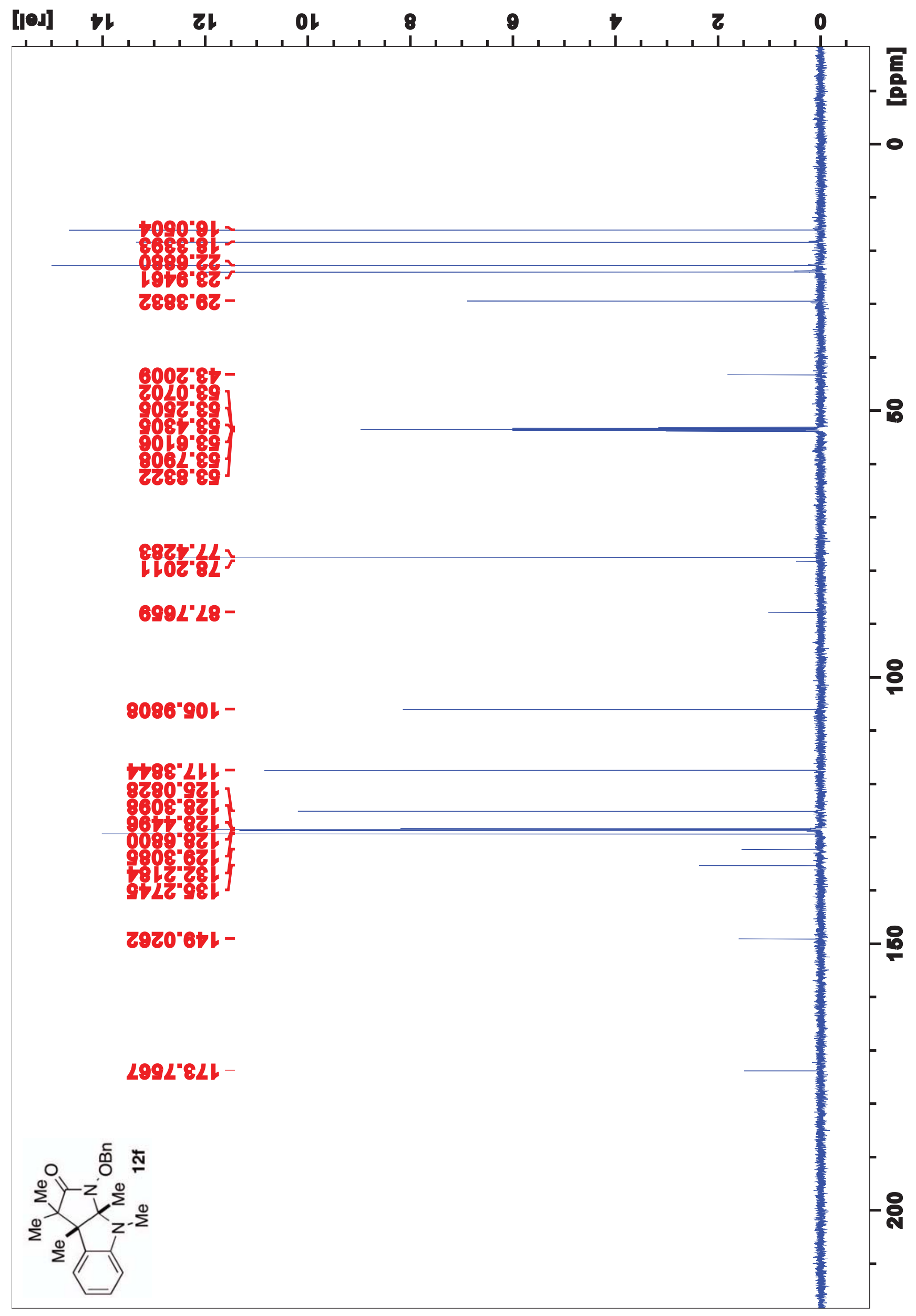




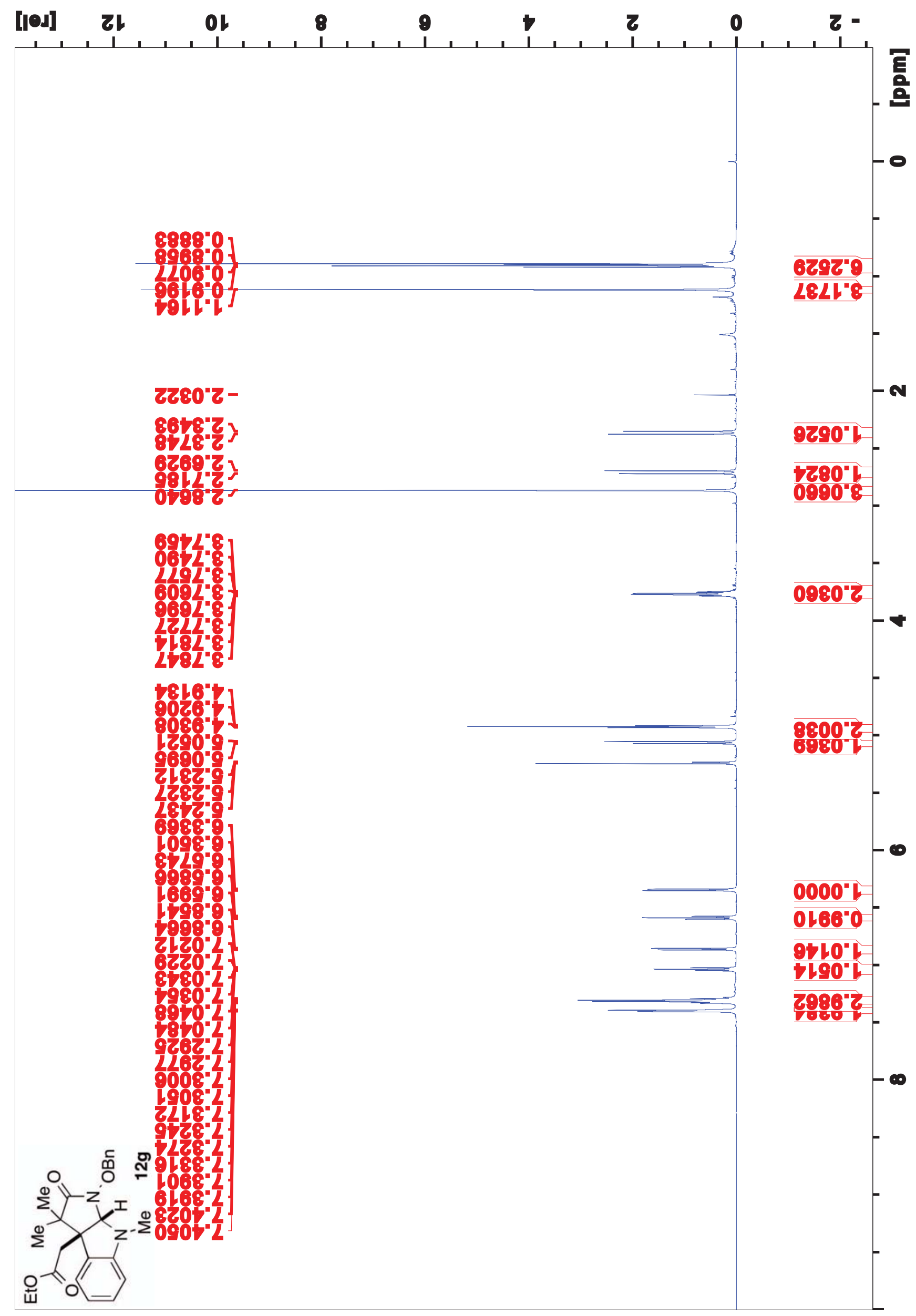




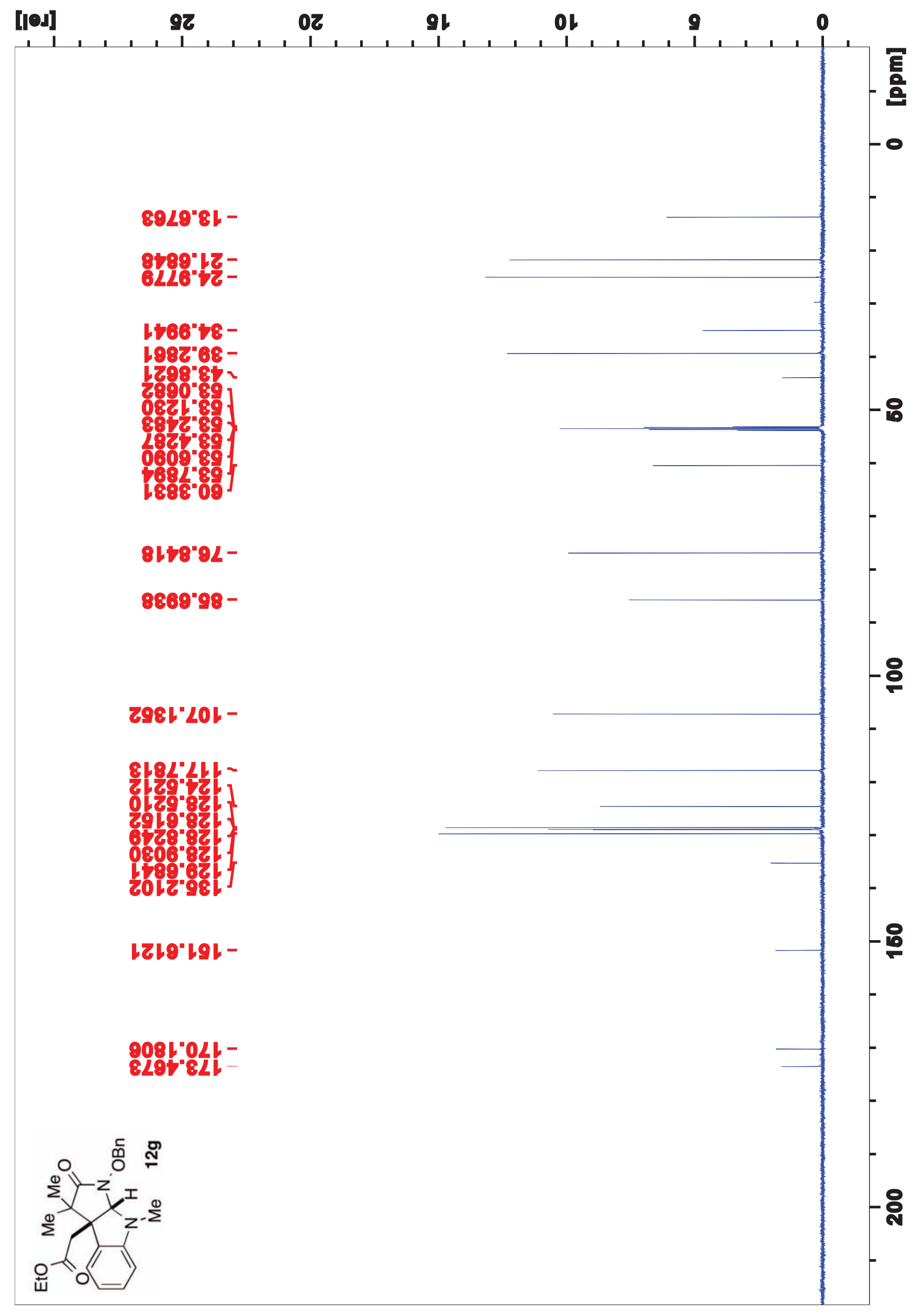









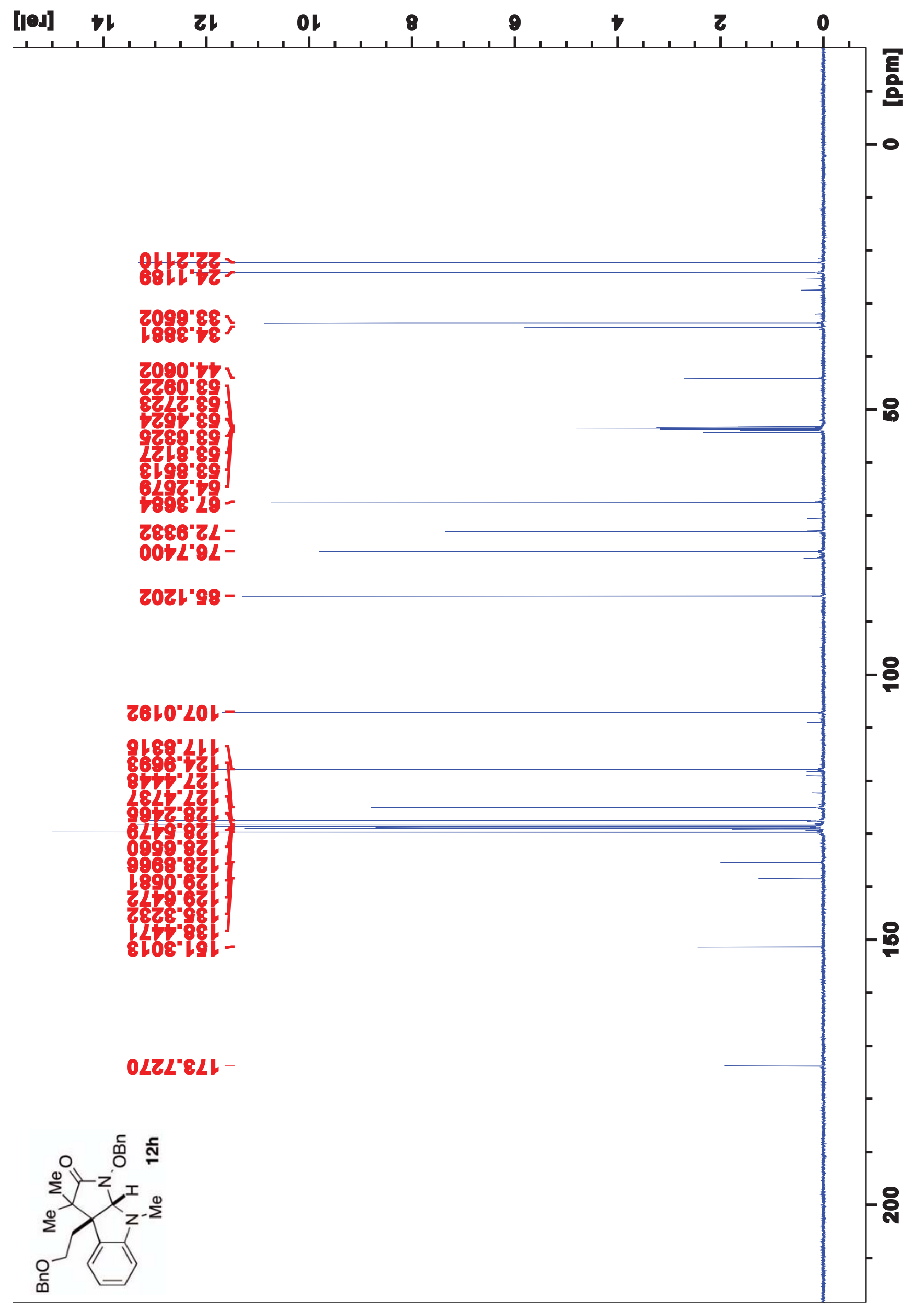




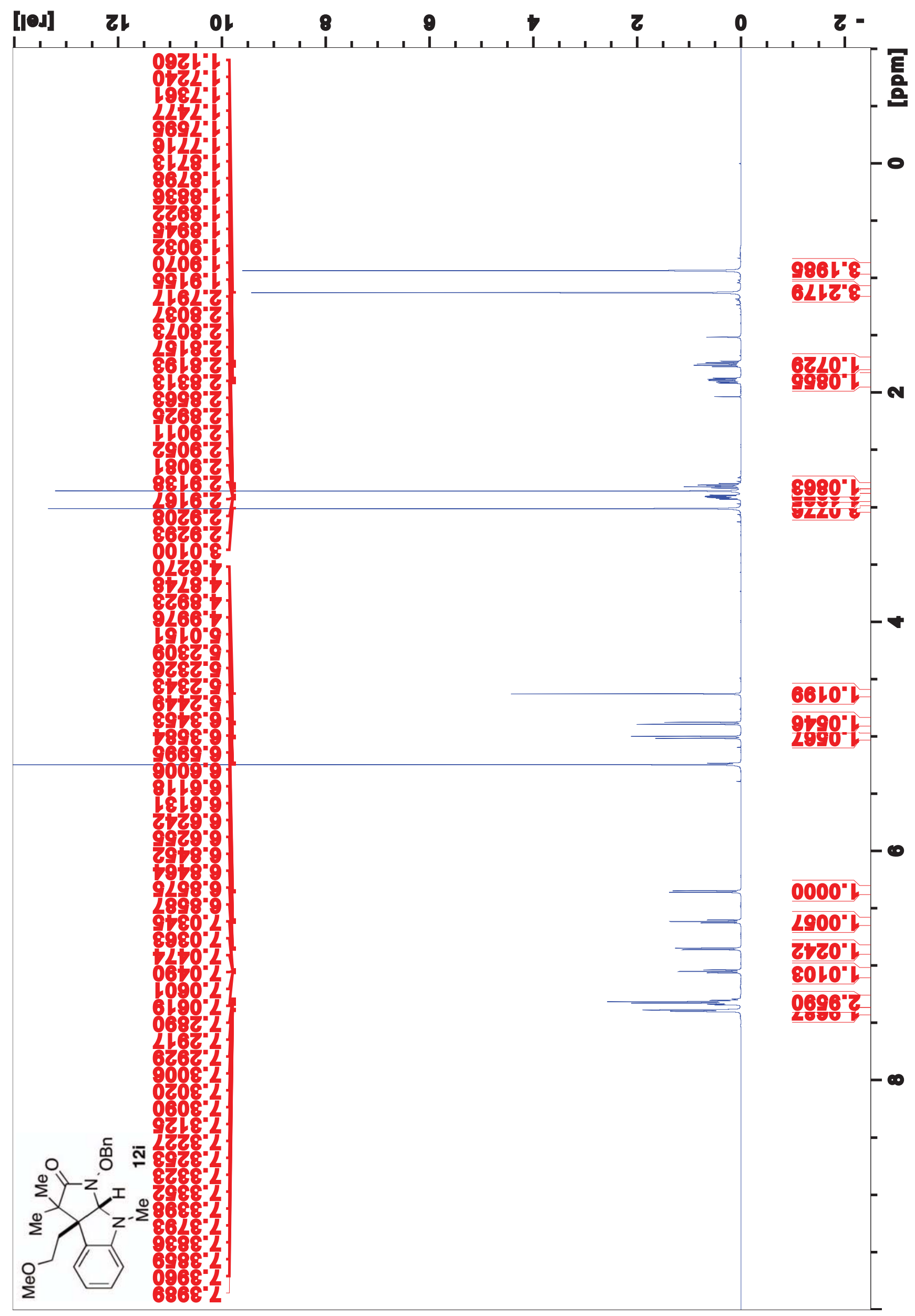




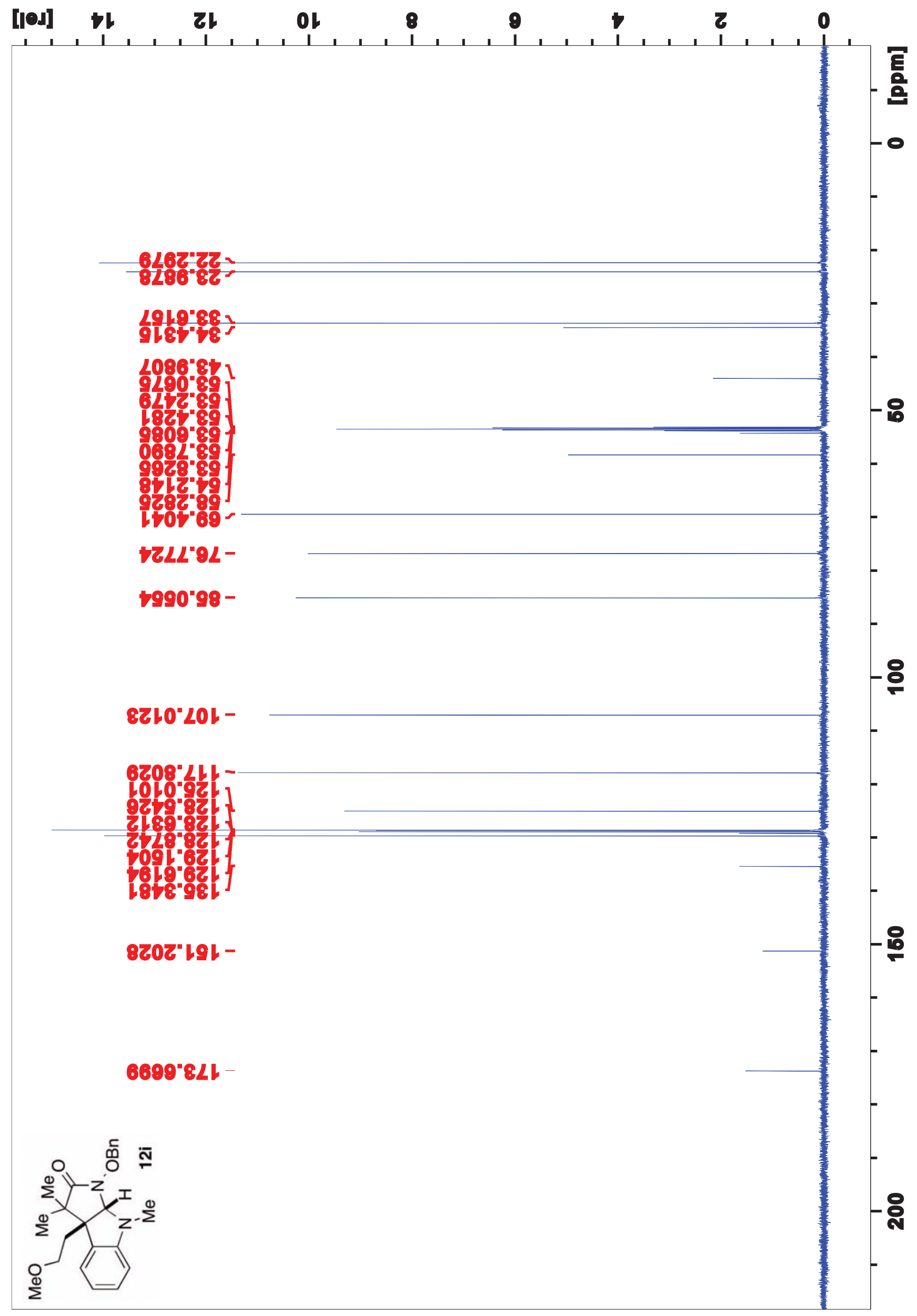




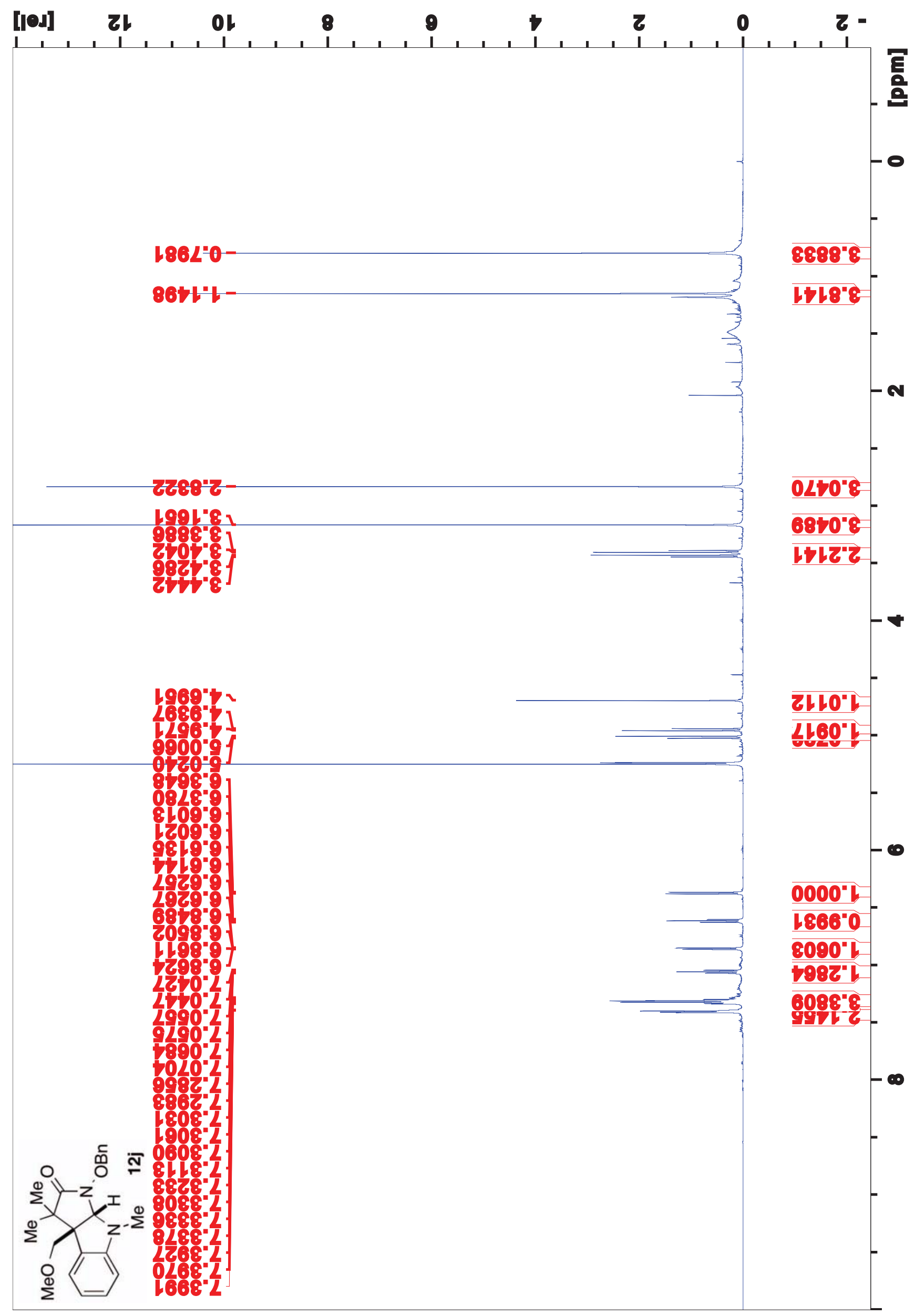




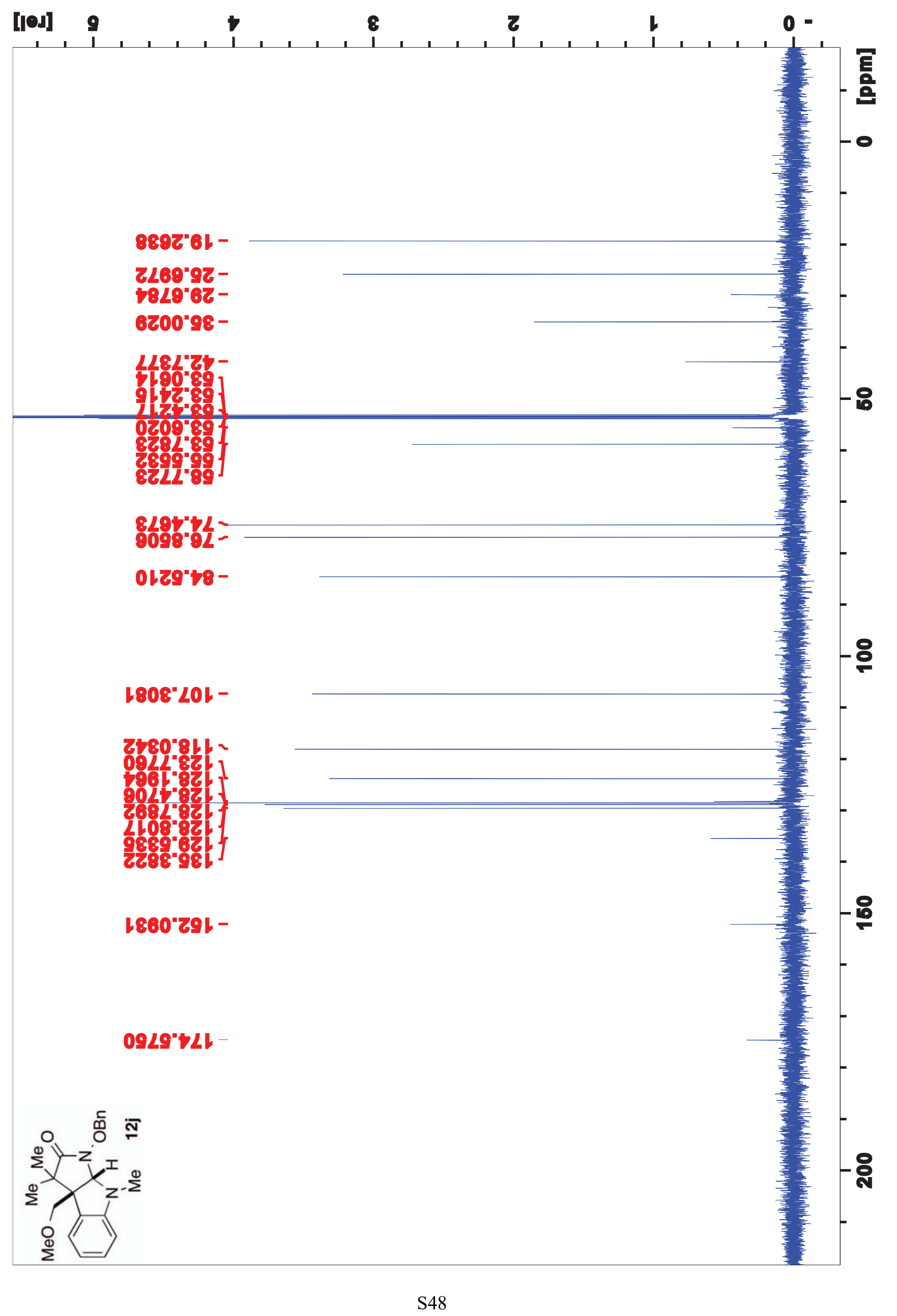




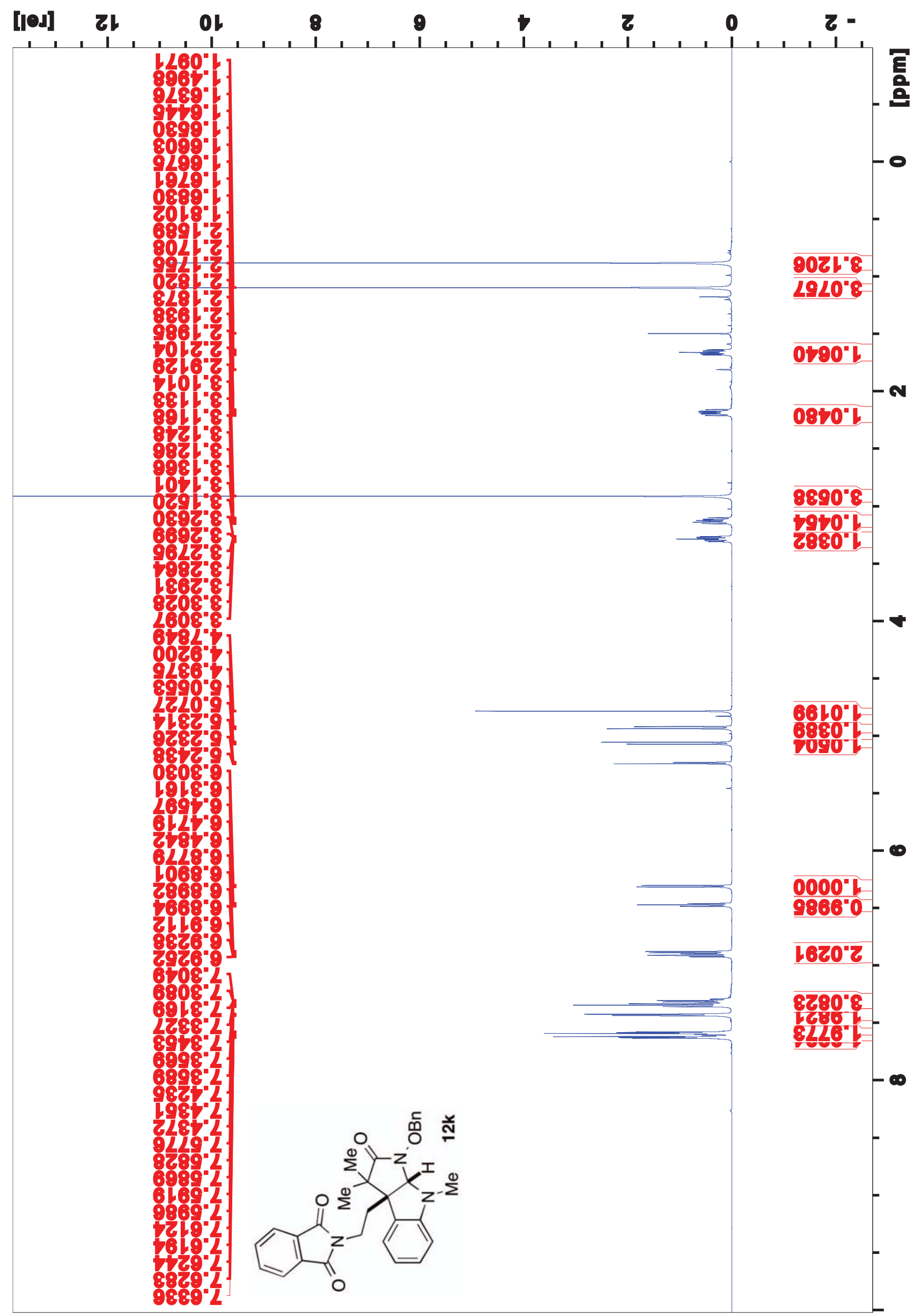




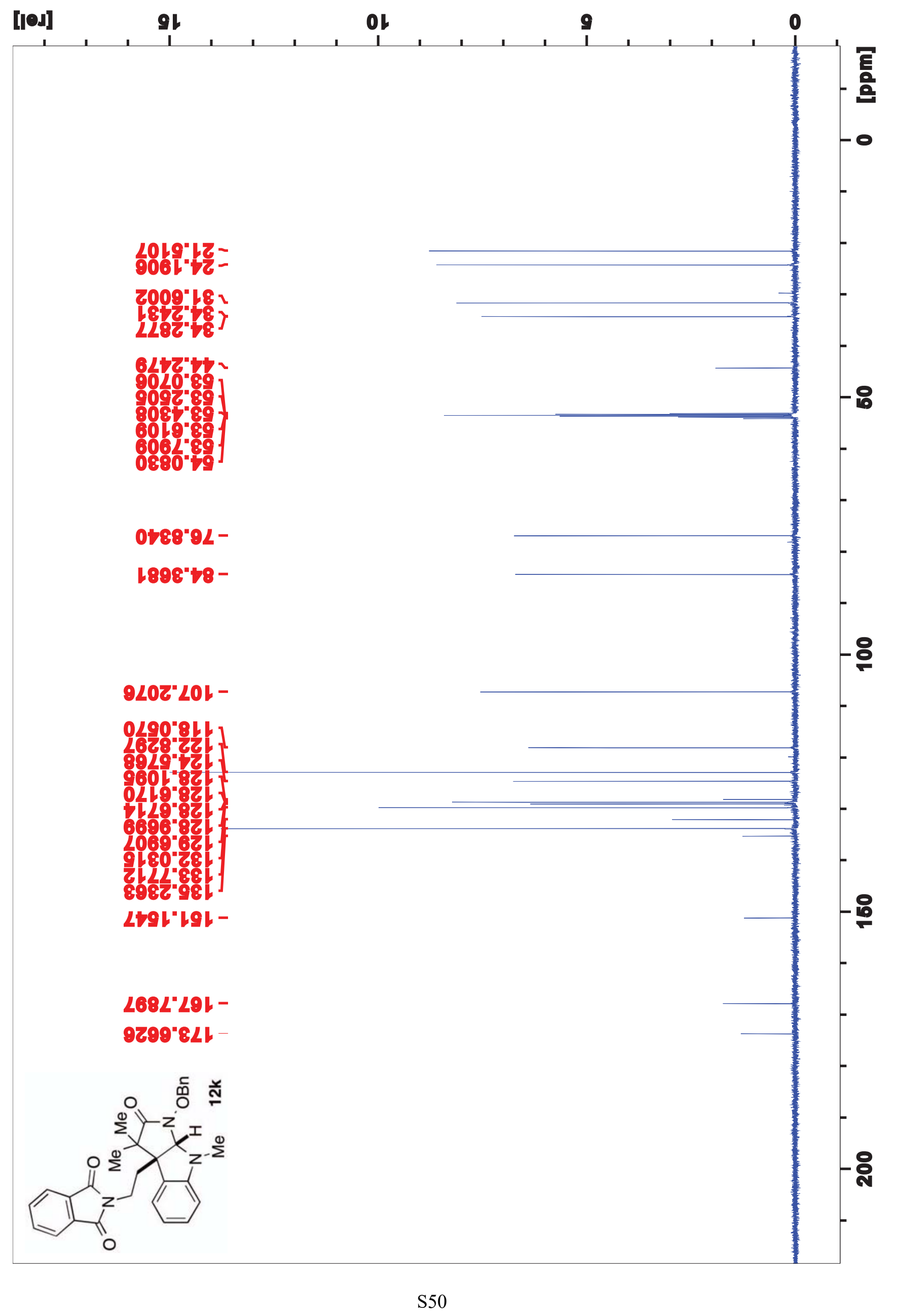




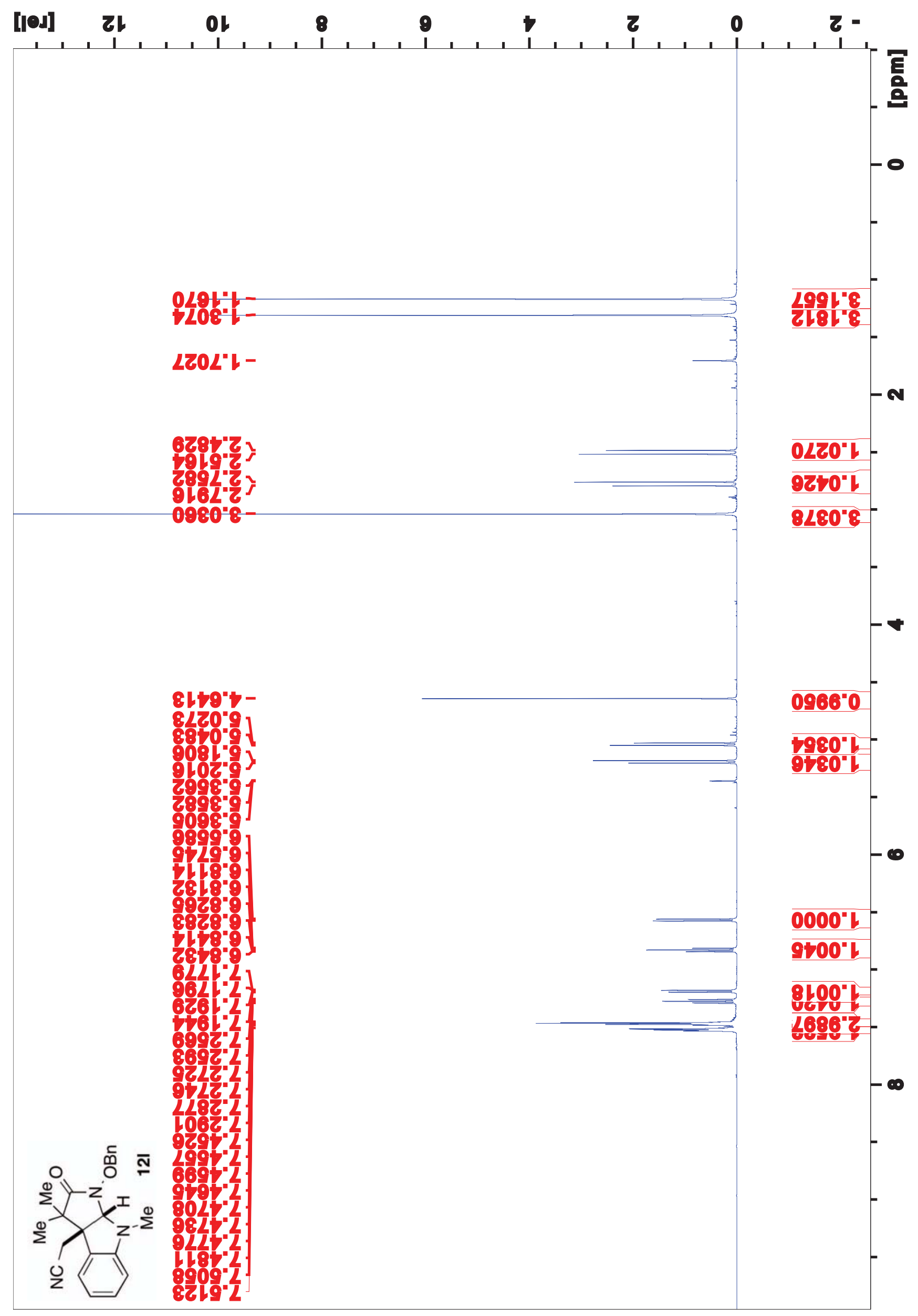




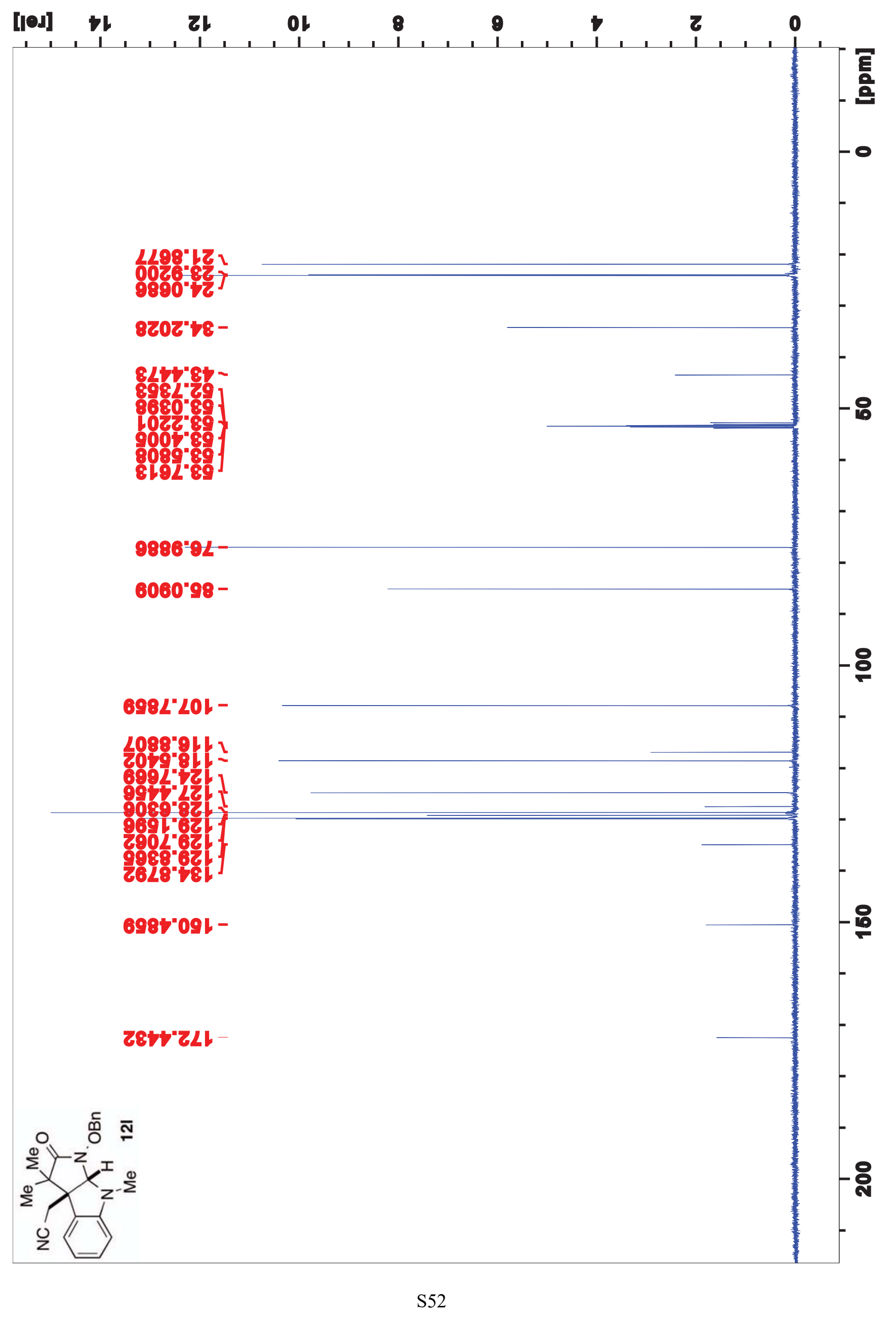




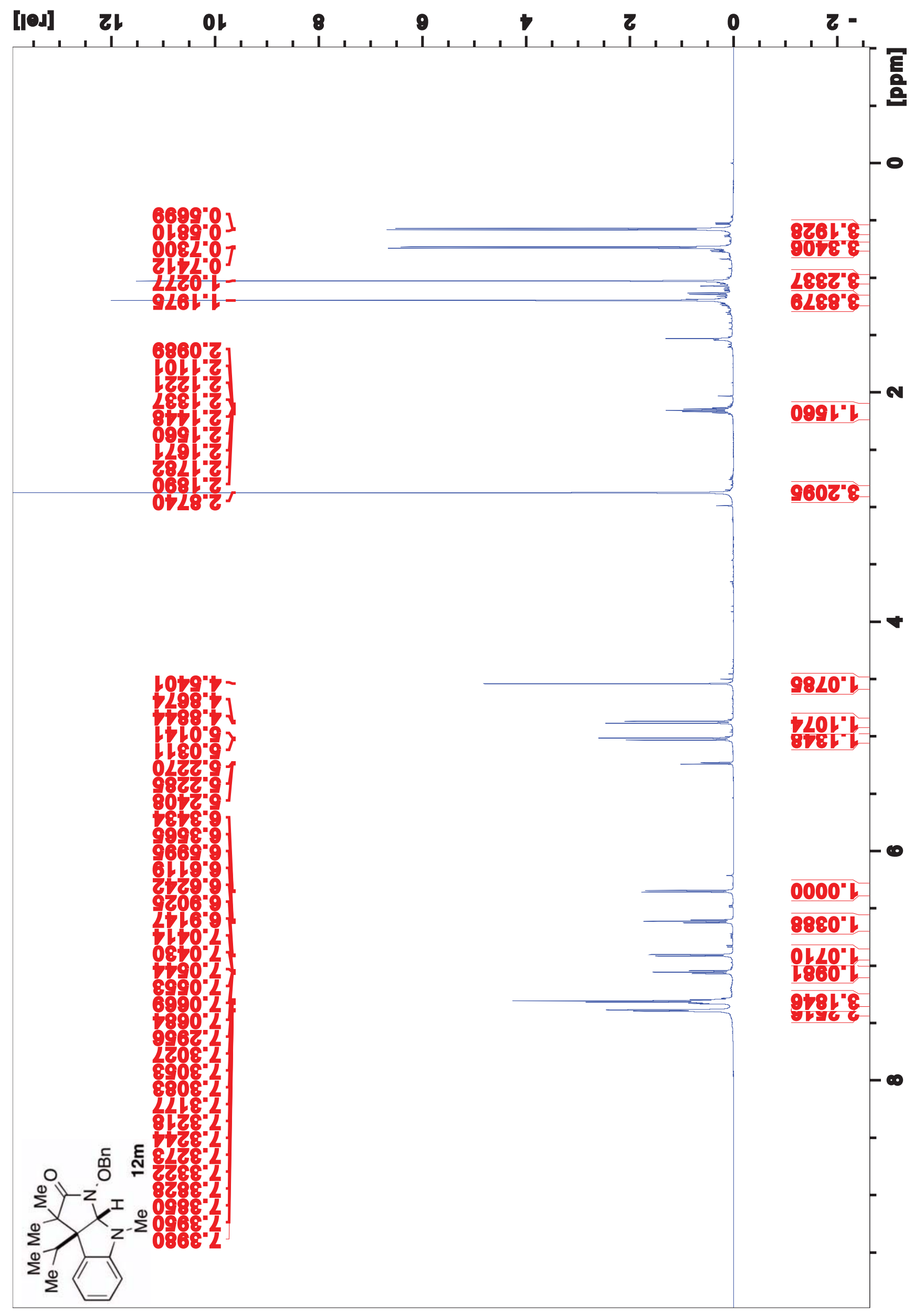




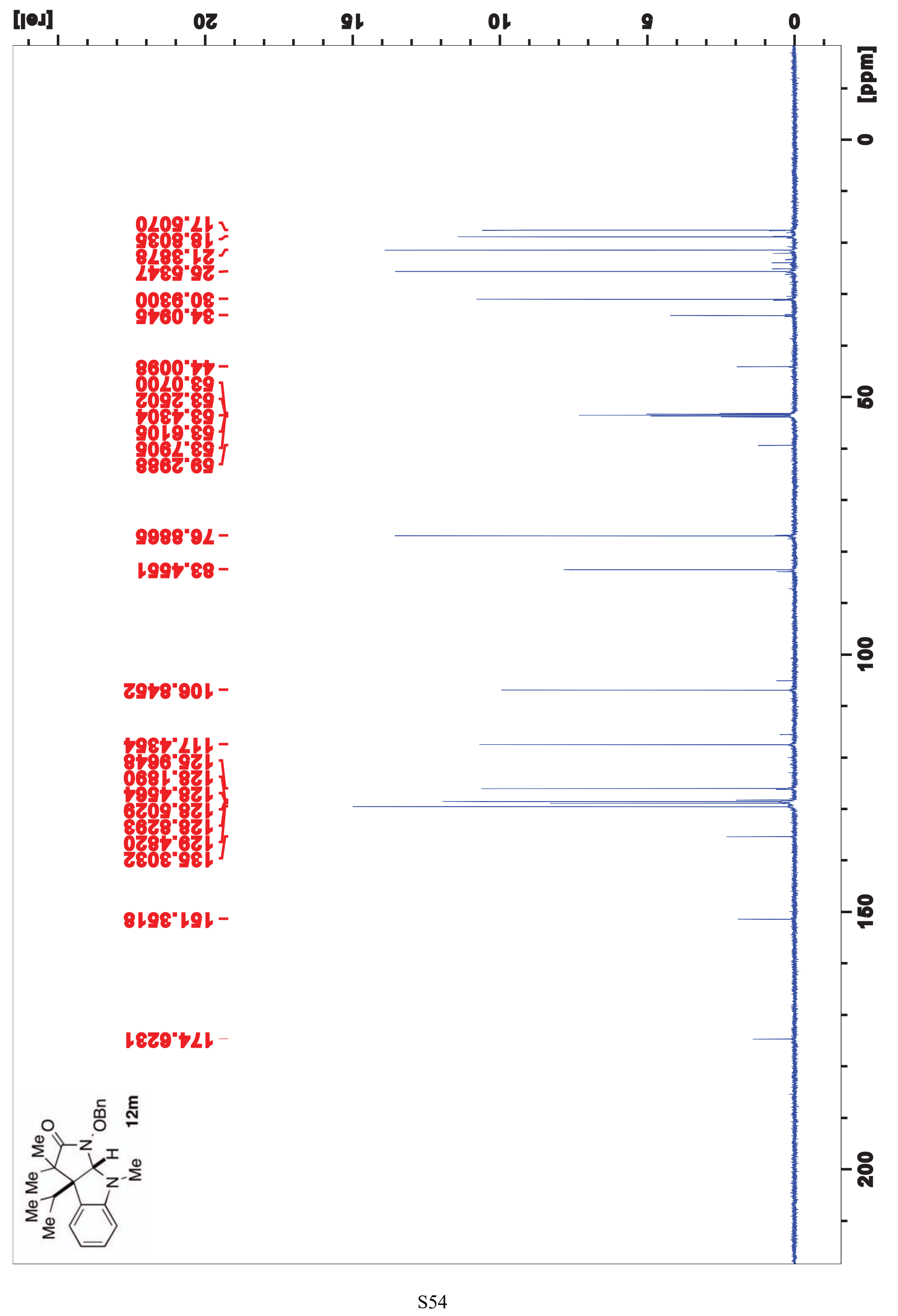




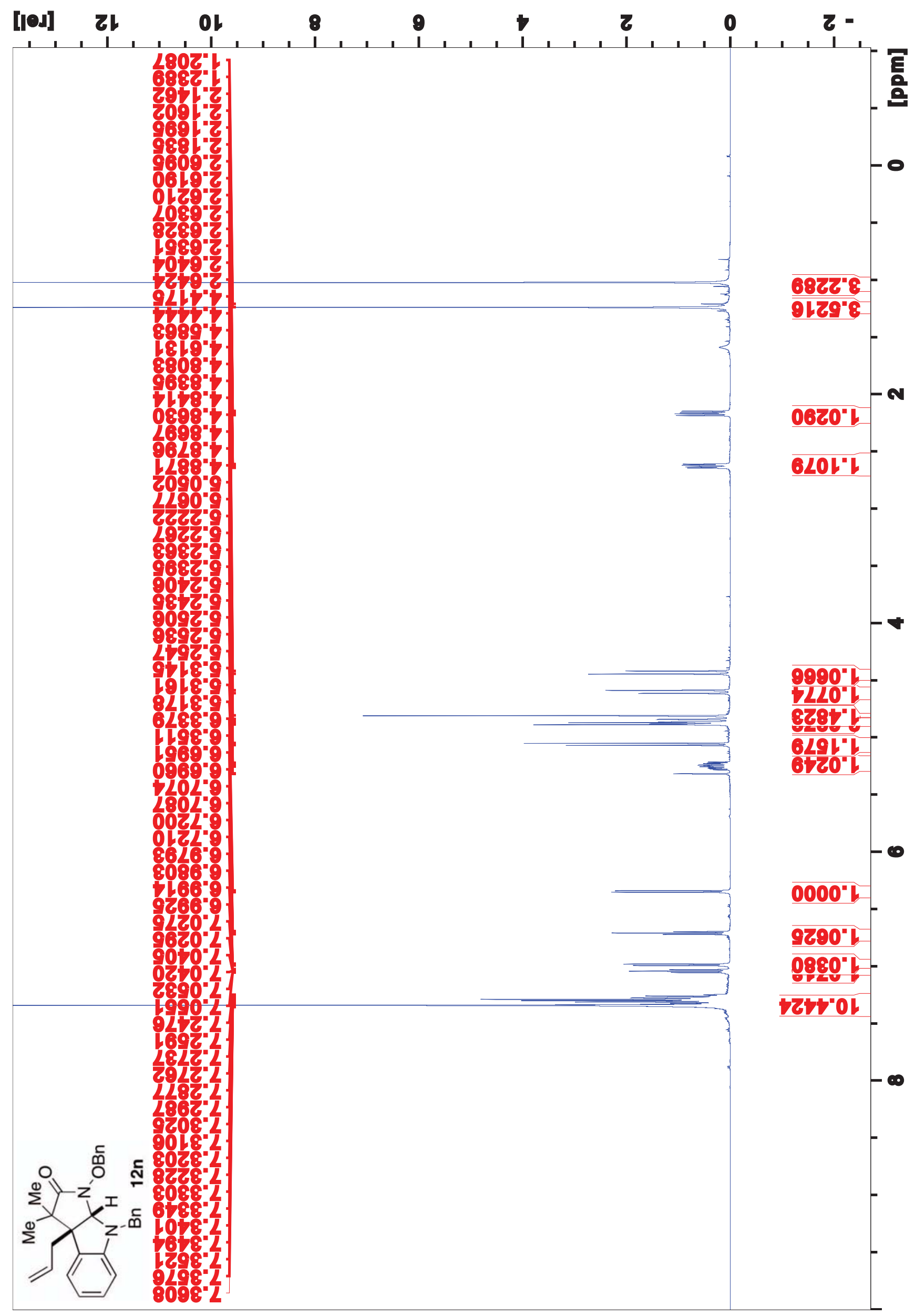




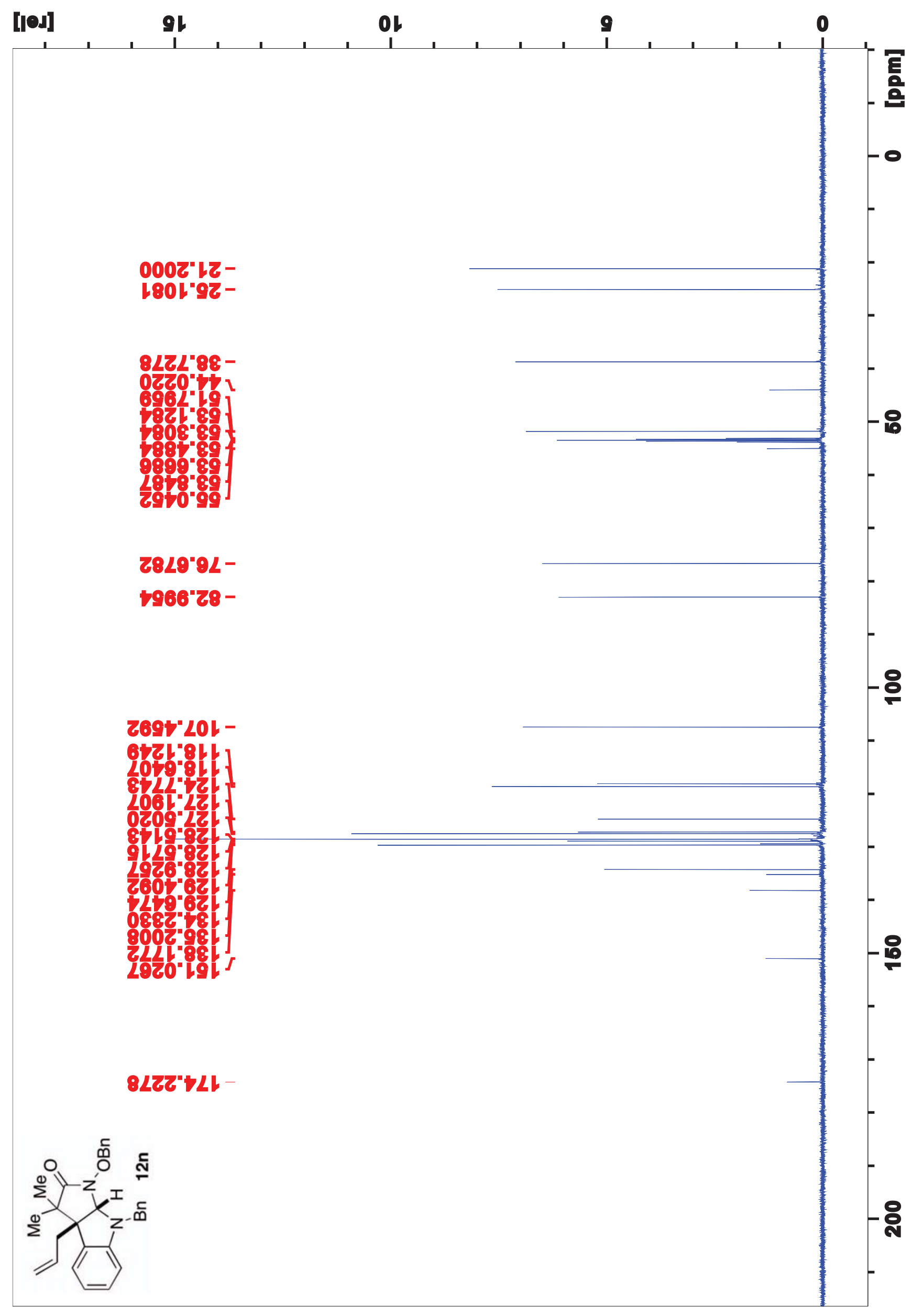




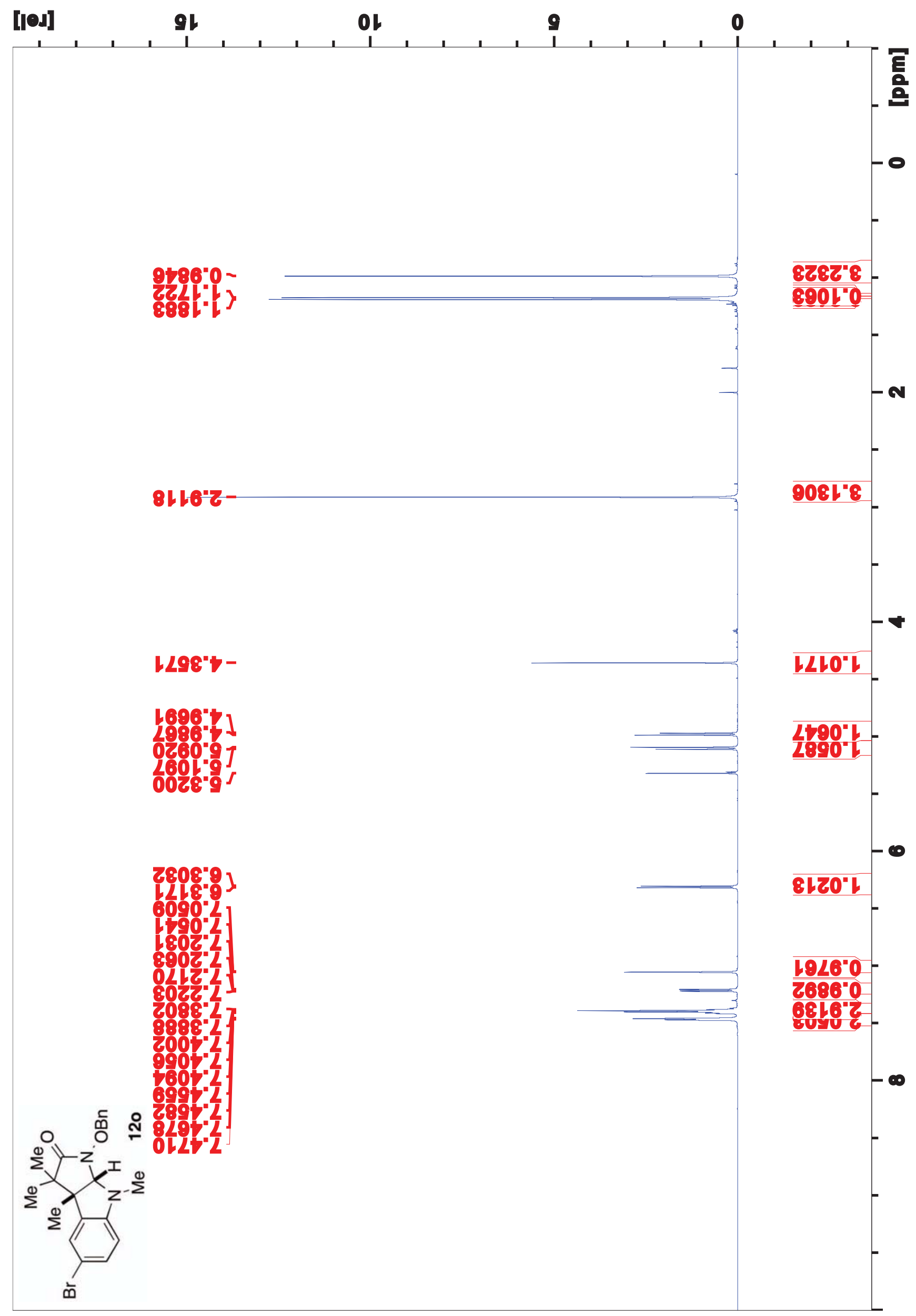




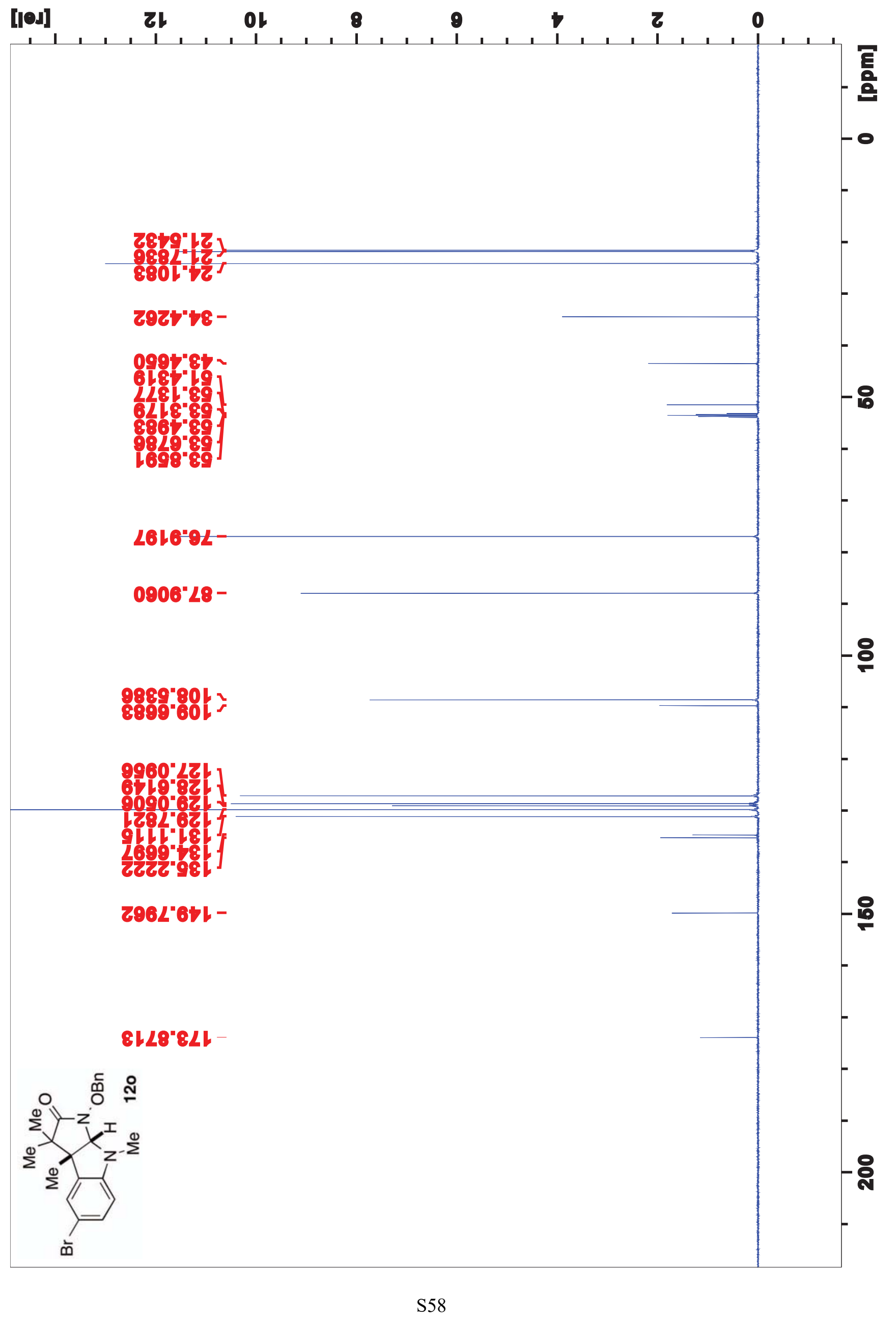




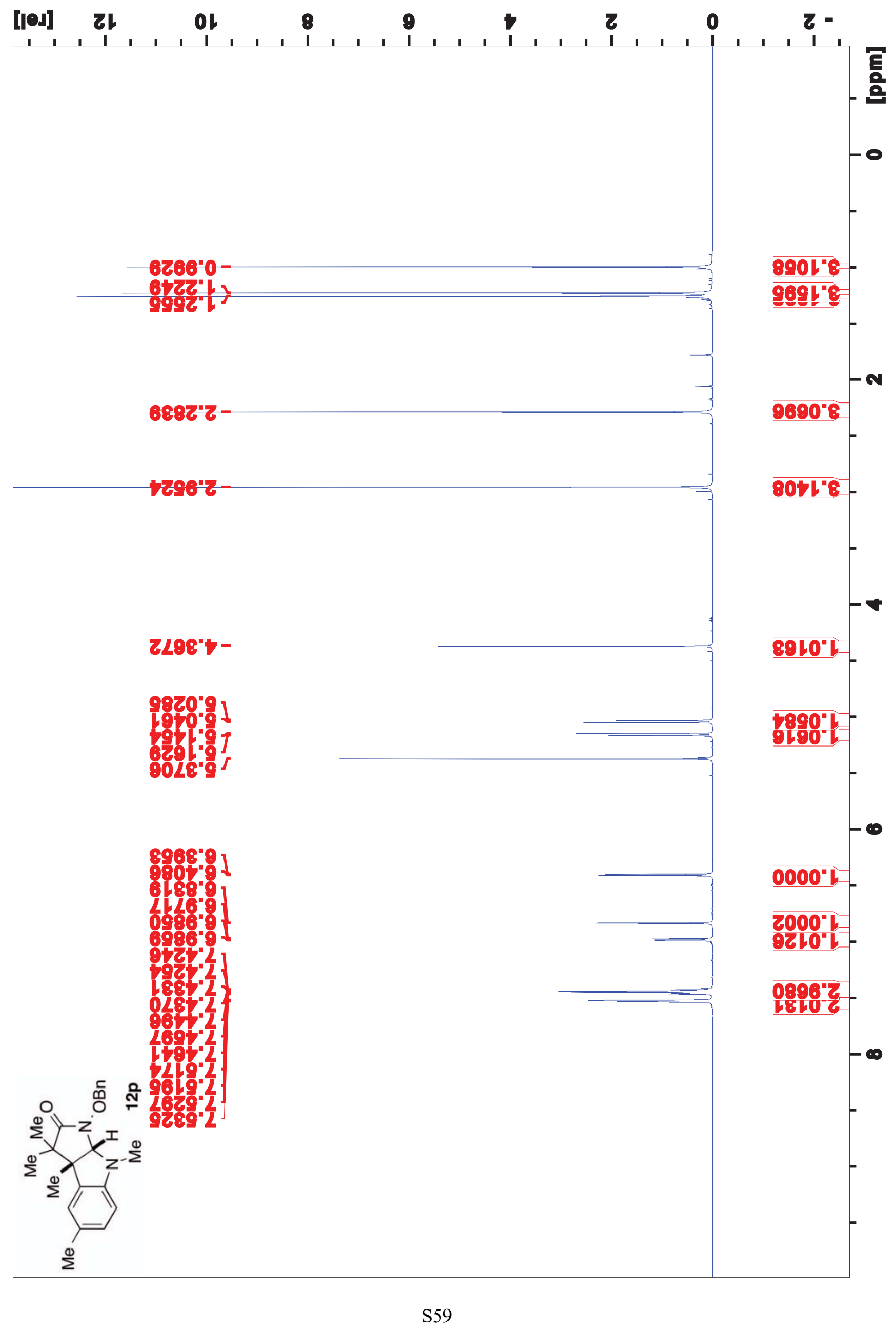




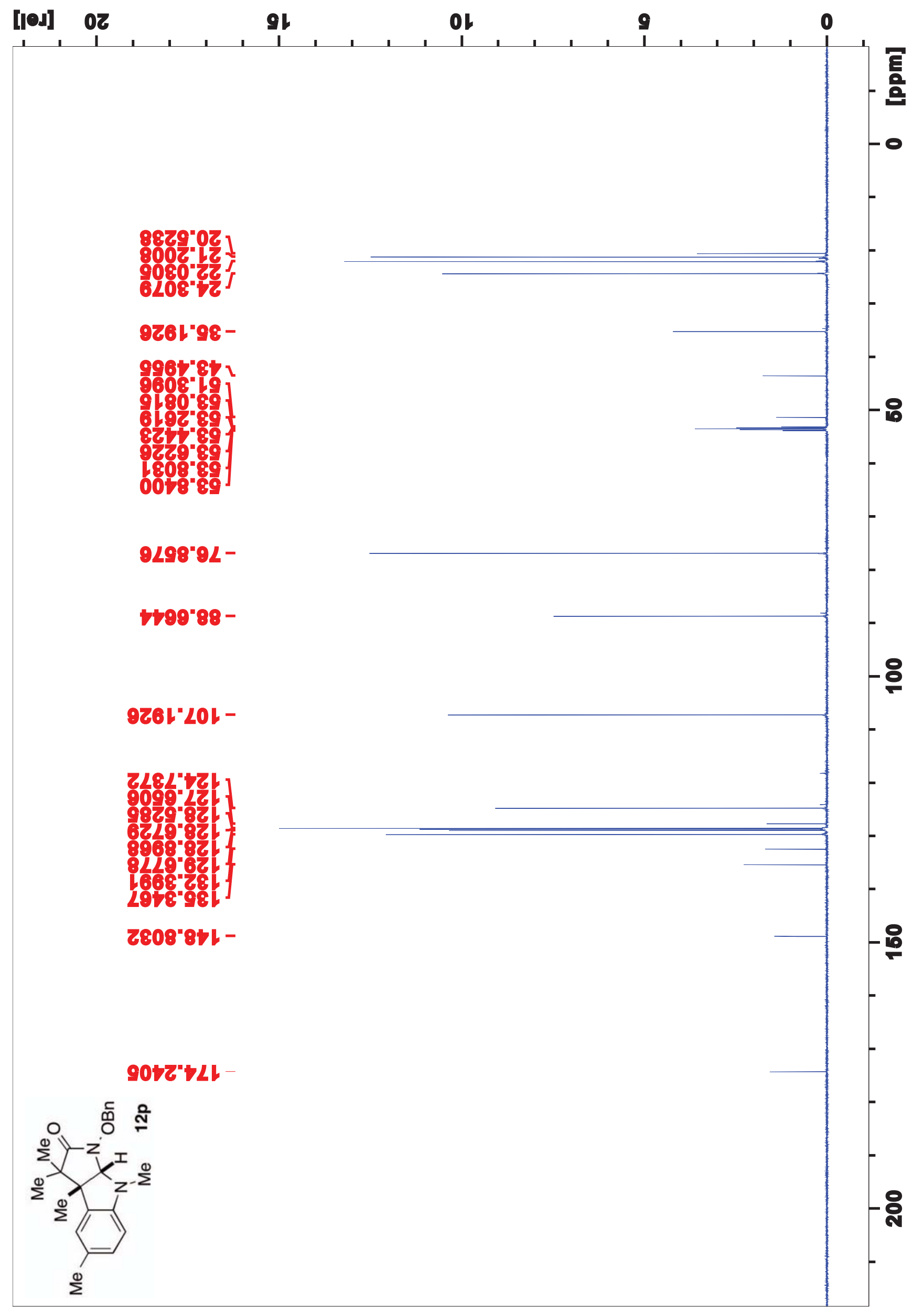




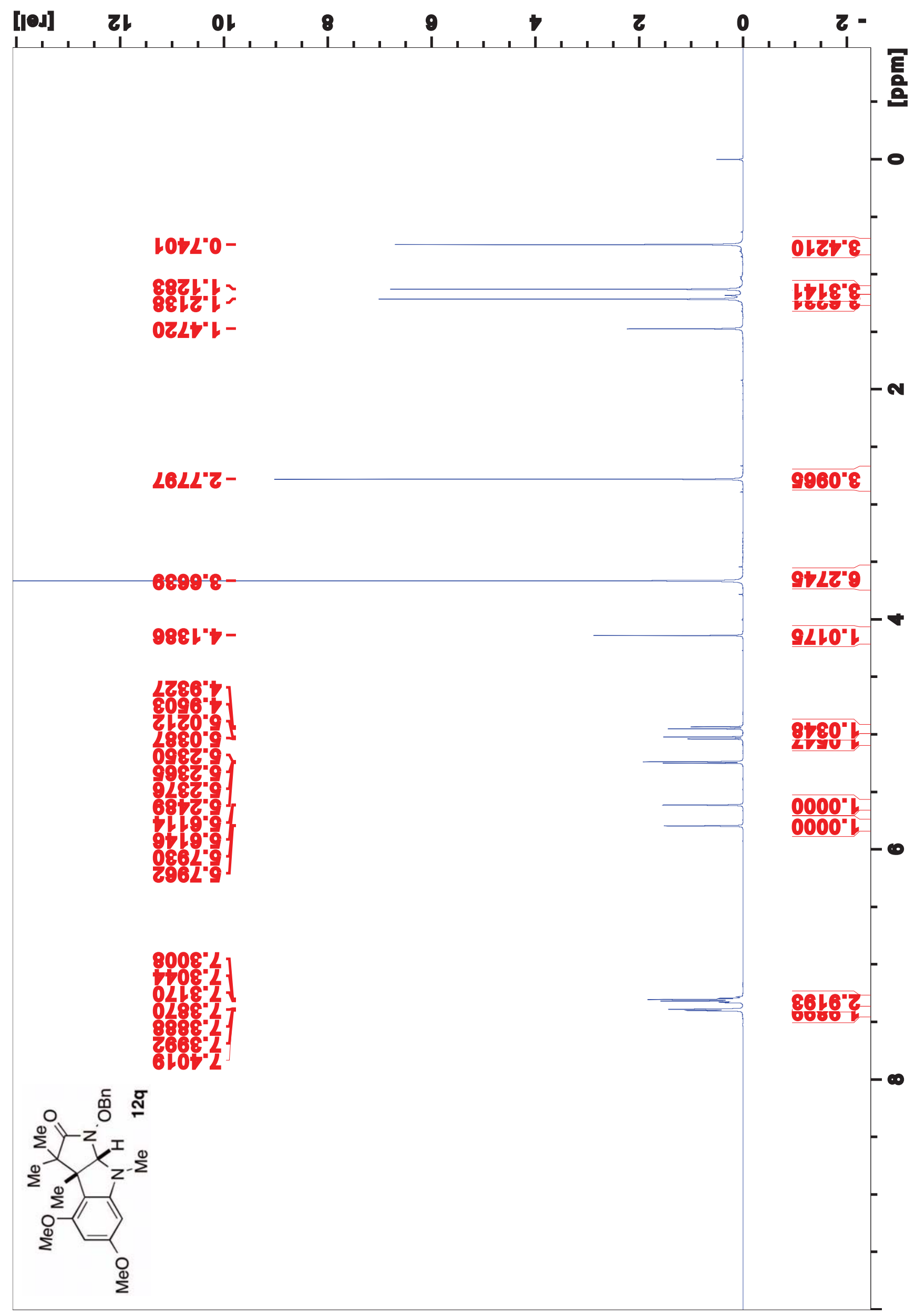




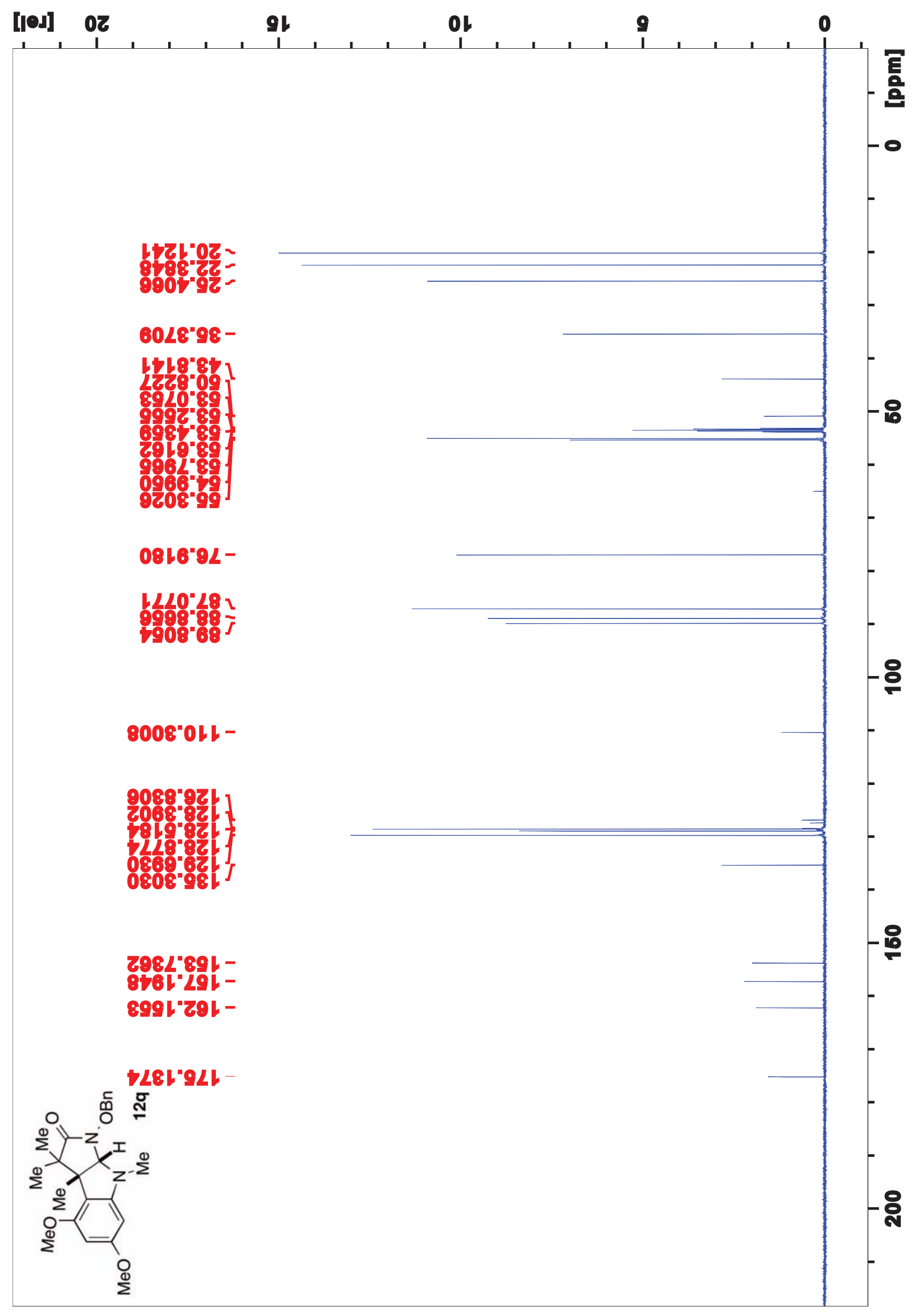




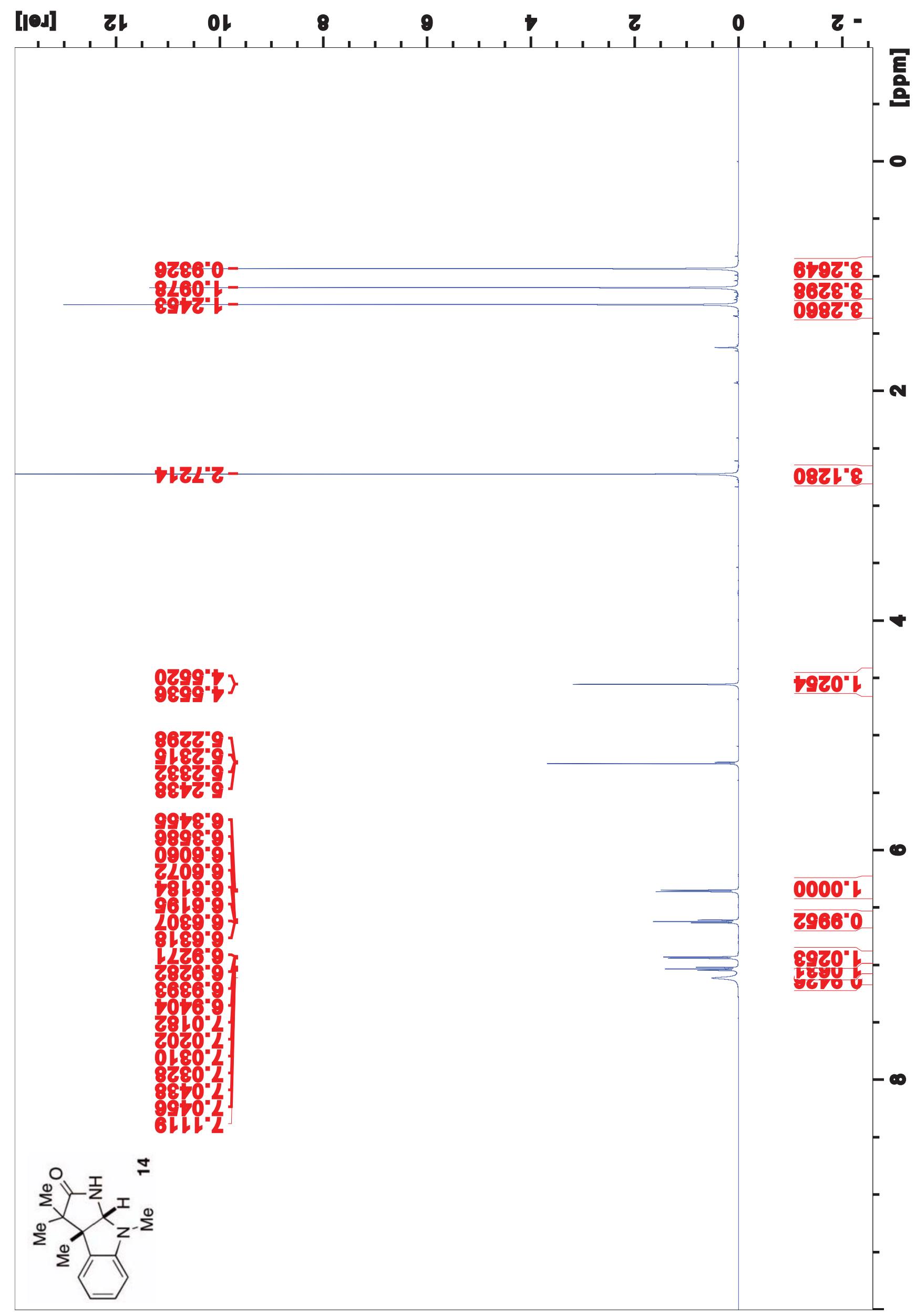




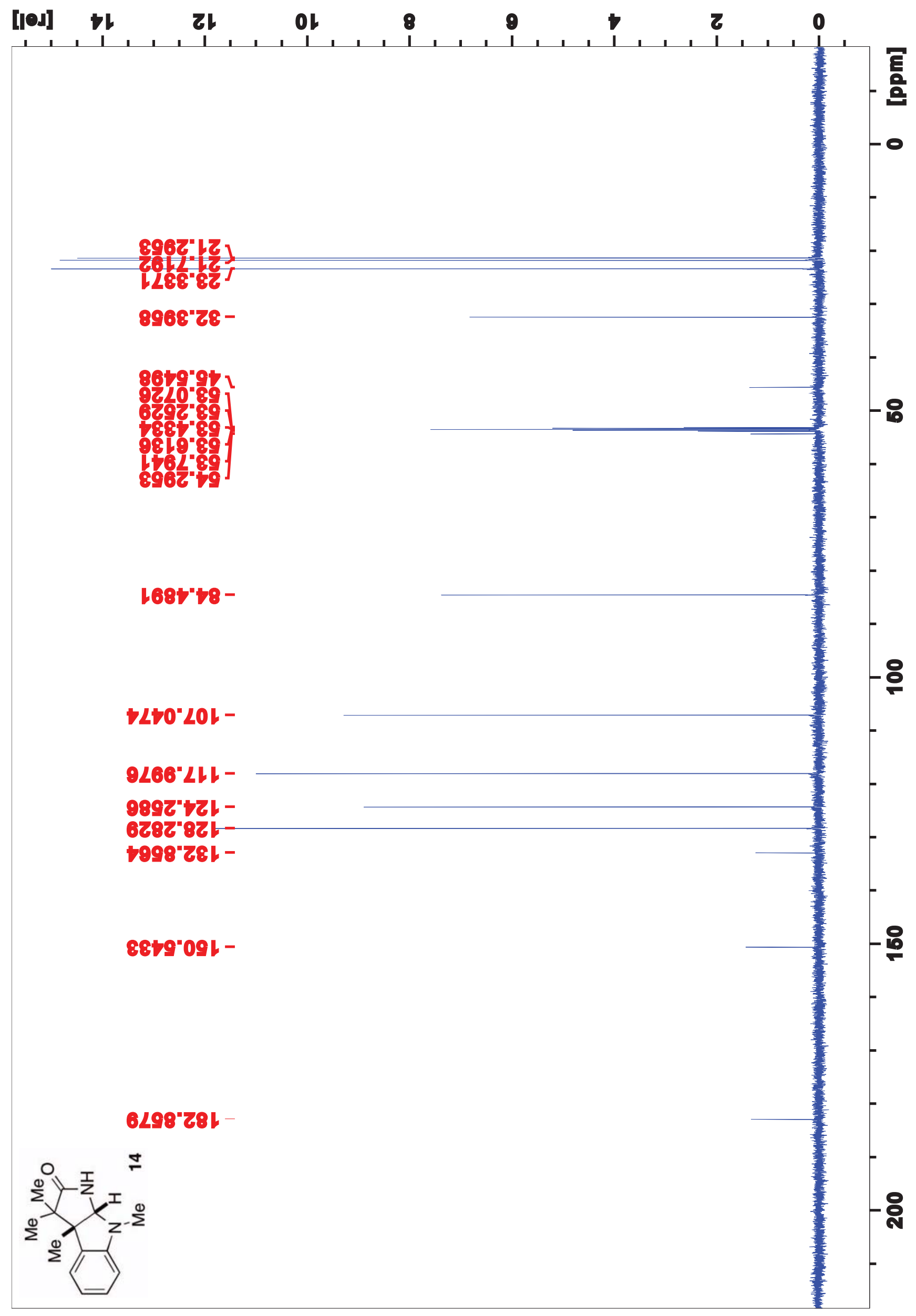




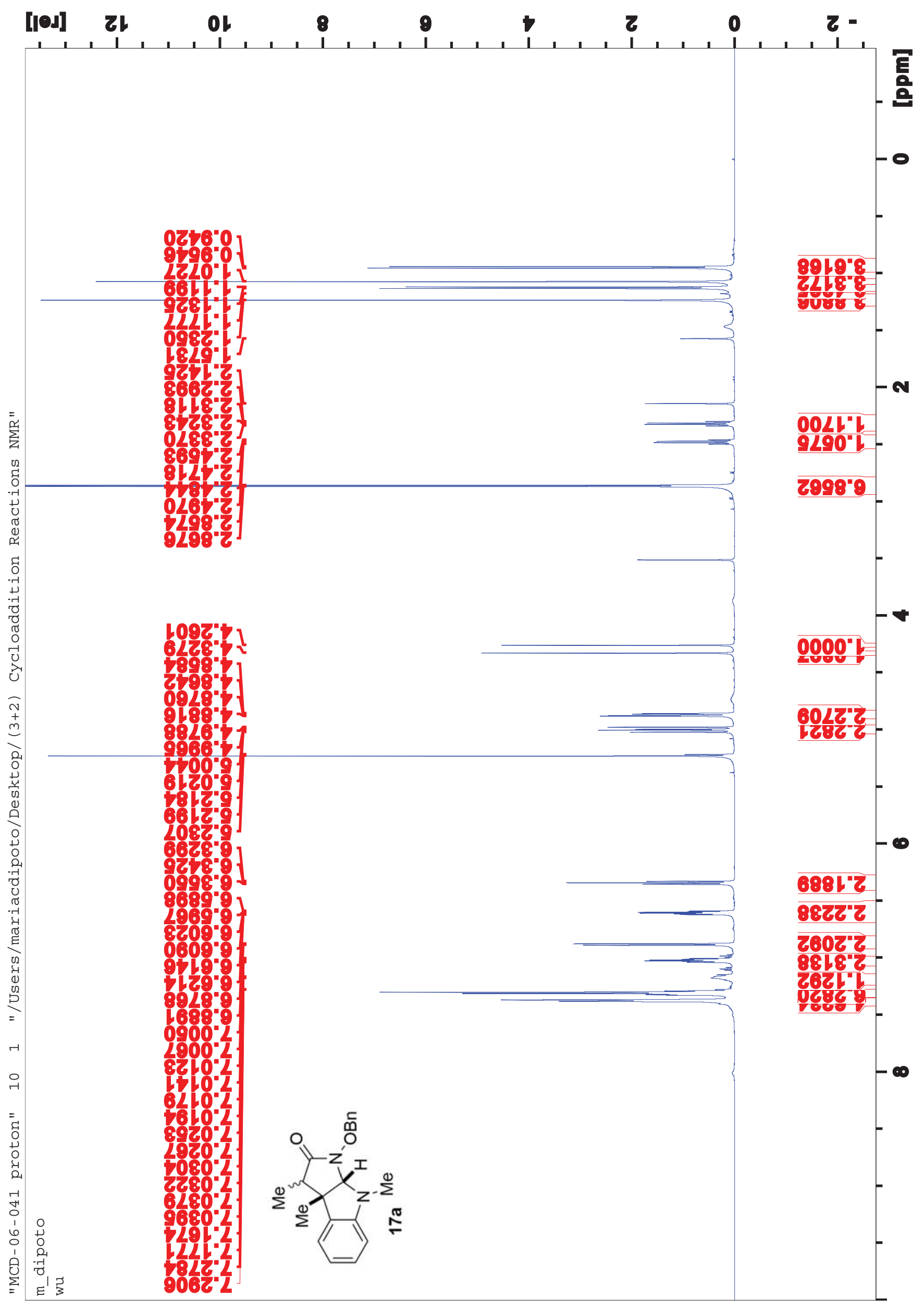




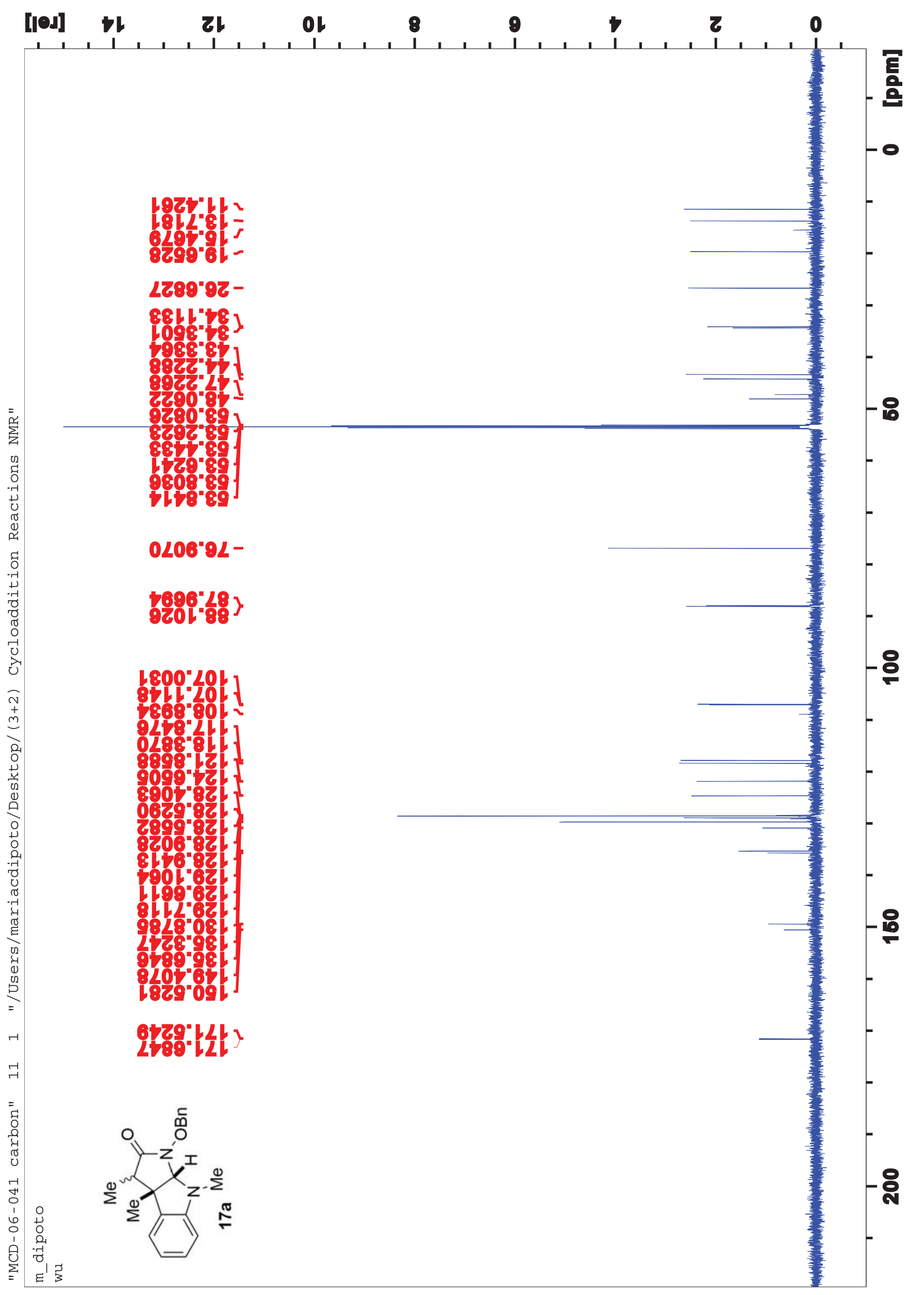




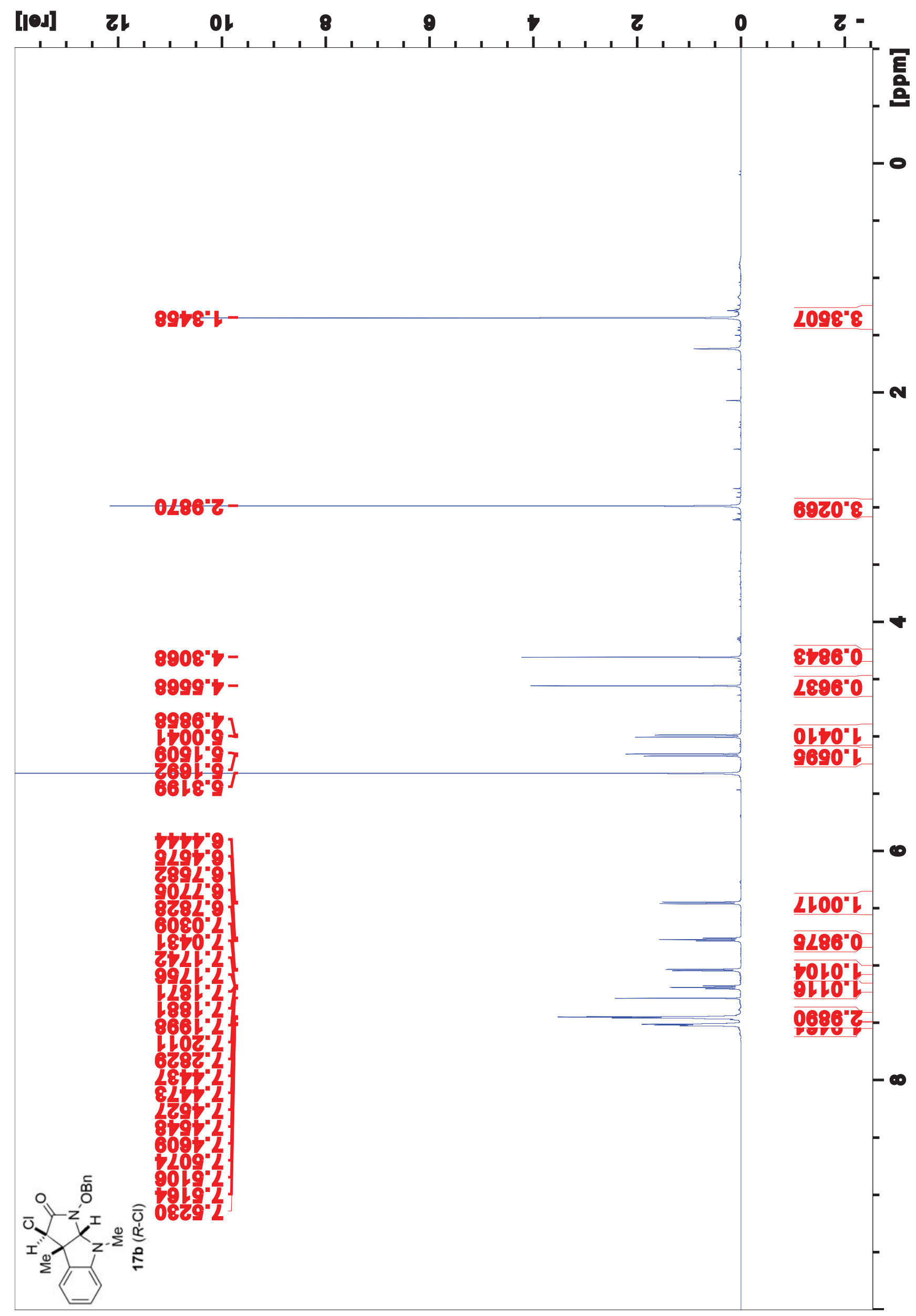




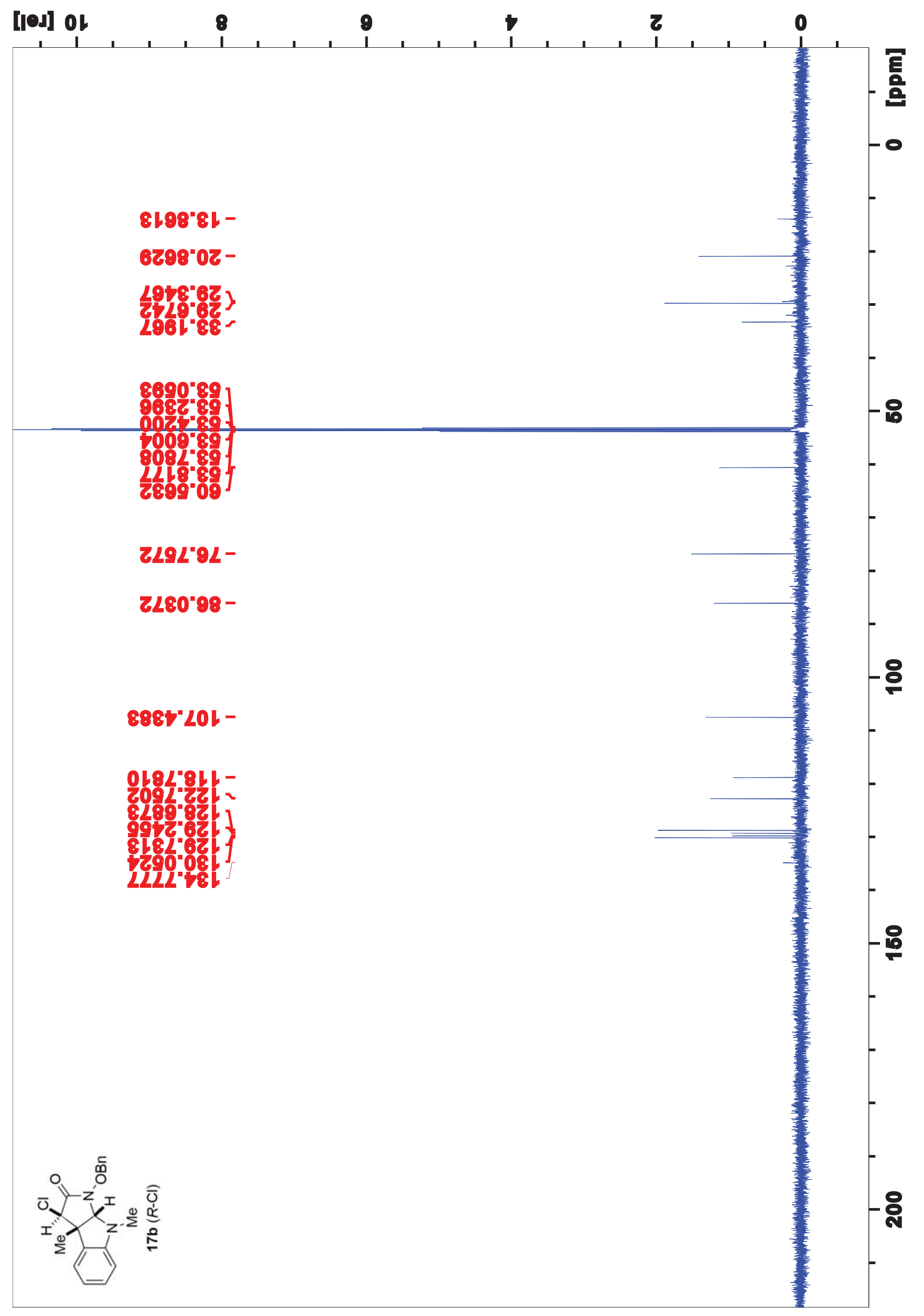




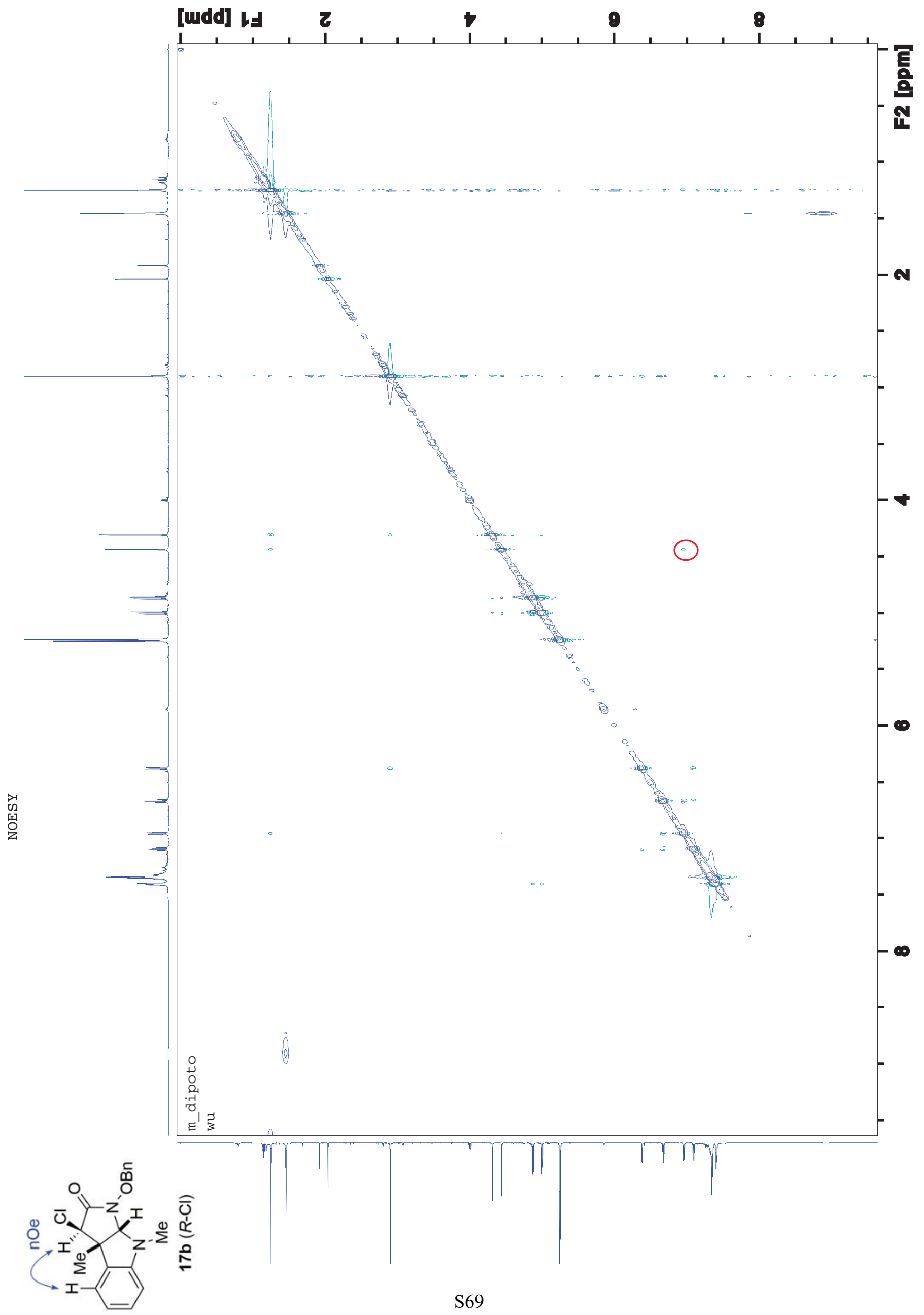




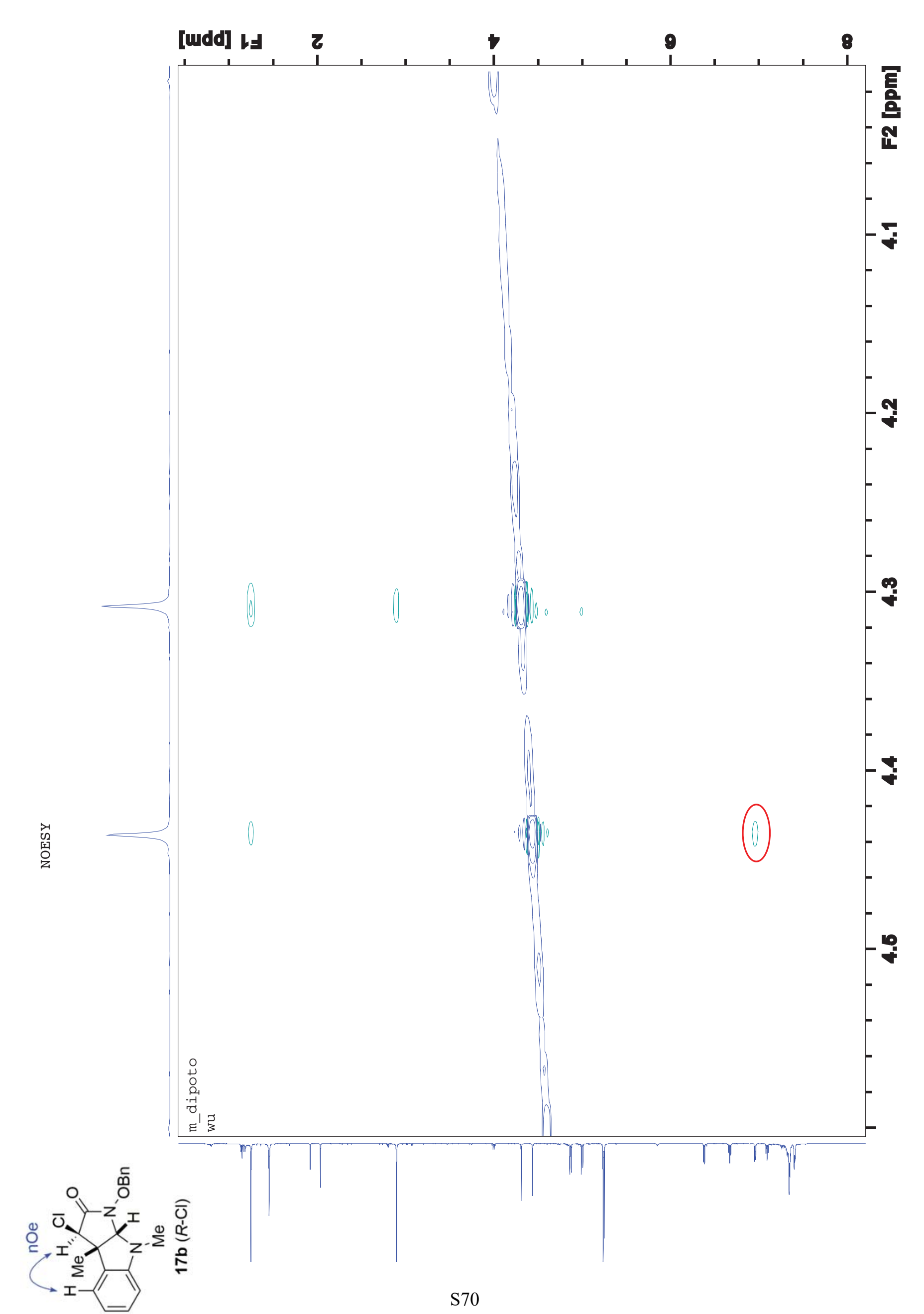




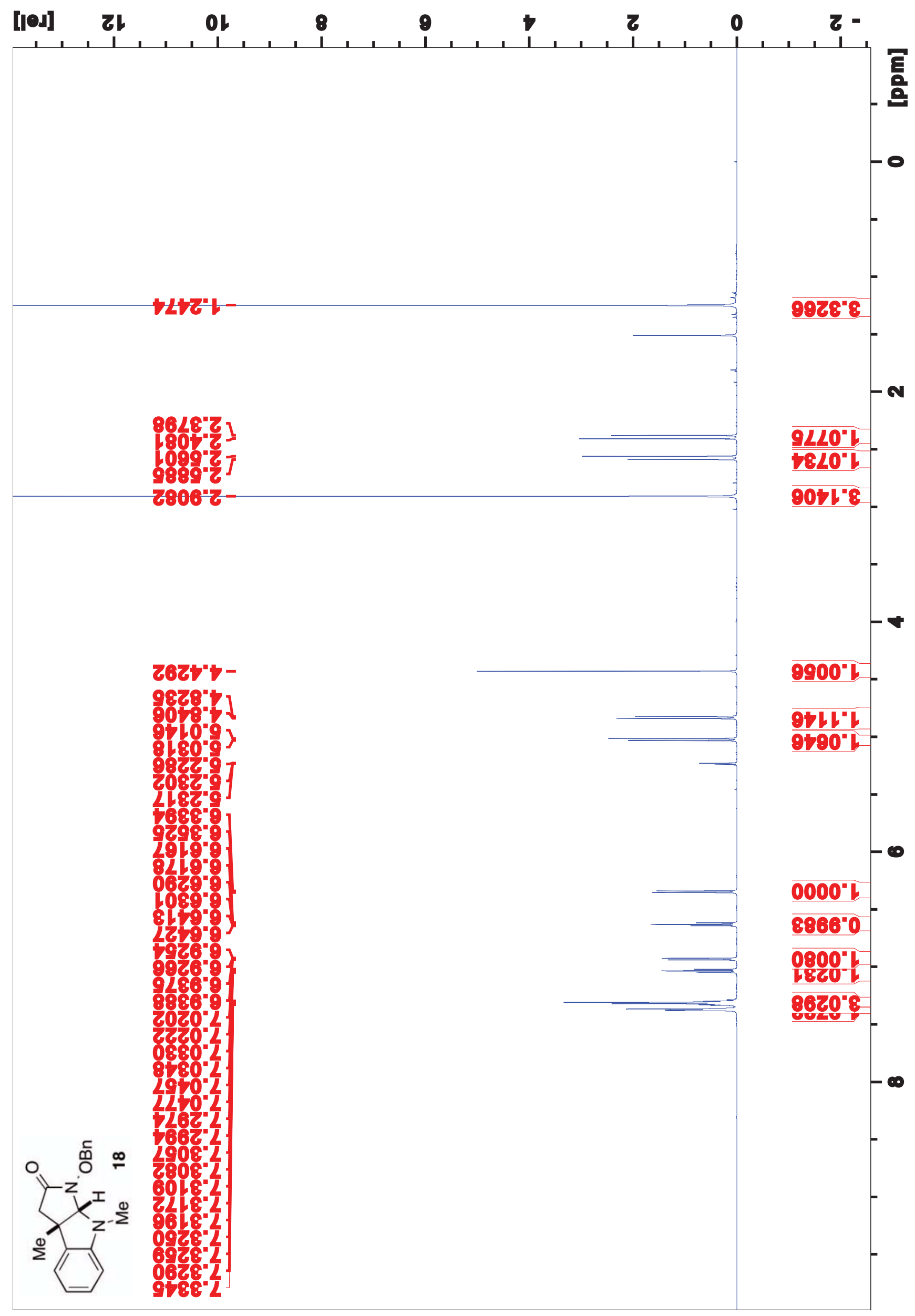




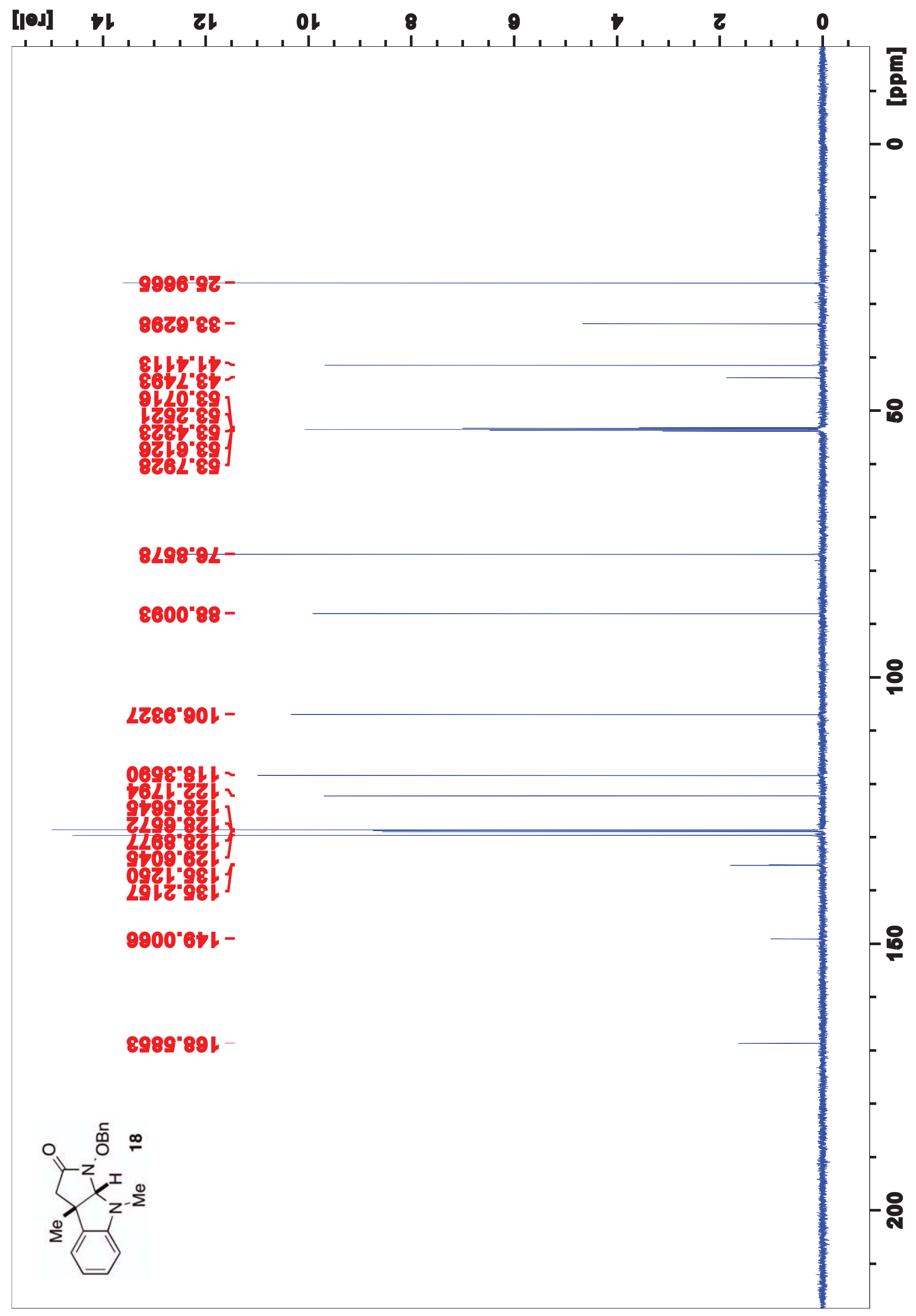

